University of Tennessee Health Science Center UTHSC Digital Commons

\title{
Investigation of a Novel Mitophagy Regulatory Pathway and Chemical Inducer
}

Jane E. Craig

University of Tennessee Health Science Center

Follow this and additional works at: https://dc.uthsc.edu/dissertations

Part of the Medical Sciences Commons

\section{Recommended Citation}

Craig, Jane E. (0000-0002-3696-347X), "Investigation of a Novel Mitophagy Regulatory Pathway and Chemical Inducer" (2020). Theses and Dissertations (ETD). Paper 528. http://dx.doi.org/10.21007/ etd.cghs.2020.0512.

This Dissertation is brought to you for free and open access by the College of Graduate Health Sciences at UTHSC Digital Commons. It has been accepted for inclusion in Theses and Dissertations (ETD) by an authorized administrator of UTHSC Digital Commons. For more information, please contact jwelch30@uthsc.edu. 


\title{
Investigation of a Novel Mitophagy Regulatory Pathway and Chemical Inducer
}

\begin{abstract}
Mitochondria are vital organelles that coordinate cellular energy homeostasis and also have important roles in cell death. Therefore, the removal of damaged or excessive mitochondria through autophagy, a process called mitophagy, is critical for maintaining proper cellular function. Much of the research elucidating the mechanism and regulation of autophagy was performed in yeast cells, so there is still a lot of mystery surrounding how mitophagy is promoted and regulated in mammals in general and in different tissues. The most well understood mechanism involved in mitophagy is the PINK1-PARKN pathway. However, studies often rely on severe mitochondrial damage using CCCP that is considered nonphysiological. To find novel regulators of mitophagy in mammalian cells, we used a previously published screen for autophagy regulators to identify the MEKK3-MEK5-ERK5 pathway as a potential regulator of mitophagy in the absence of exogenous damage. Here, I provide evidence that genetic or pharmacological inhibition of the MEKK3-MEK5-ERK5 pathway increases mitochondrial content by reducing lysosome-mediated degradation of mitochondria under basal conditions. I confirm that the MEKK3-MEK5-ERK5 pathway is not required for non-selective bulk autophagy, damage-induced mitophagy, or restraint of mitochondrial biogenesis, confirming a selective role in basal mitochondrial degradation. Furthermore, inhibition of MEK5 or ERK5 in erythrocytes leads to defective mitochondrial elimination and impaired erythrocyte differentiation. In addition to finding a novel mitophagy promoting pathway, we also worked to discover a novel mitophagy inducing compound. There are several different mechanisms for mitophagy induction, so there is considerable interest in the field in identifying novel chemical modulators that promote the efficient removal of mitochondria through different pathways.
\end{abstract}

Document Type

Dissertation

Degree Name

Doctor of Philosophy (PhD)

Program

Biomedical Sciences

Research Advisor

Malia B. Potts, PhD

Keywords

Biology, Cellular biology

Subject Categories

Medical Sciences | Medicine and Health Sciences 
DOCTOR OF PHILOSOPHY DisSERTATION

\section{Investigation of a Novel Mitophagy Regulatory Pathway and Chemical Inducer}

Author:

Jane E. Craig
Advisor:

Malia B. Potts, $\mathrm{PhD}$

A Dissertation Presented for The Graduate Studies Council of

The University of Tennessee Health Science Center

in Partial Fulfillment of the Requirements for the

Doctor of Philosophy degree from

The University of Tennessee

in

Biomedical Sciences: Cancer and Developmental Biology

College of Graduate Health Sciences 
Copyright (C) 2020 by Jane E. Craig. All rights reserved. 


\section{DEDICATION}

This dissertation is dedicated my family. I would like to thank my husband, Andrew, for his perpetual support and encouragement; I could not have made it without you. I would also like to thank my parents, Kathy and Joe, and my brother, Daniel, for being my biggest cheerleaders and always believing in me. 


\section{ACKNOWLEDGEMENTS}

I owe a great deal of gratitude to my mentor, Dr. Malia Potts, for all the time and effort she devoted to the training I was given. Her perspective and guidance were invaluable.

I owe my fellow lab mates a word of thanks for all the discussion, advice, and questions over the years. I would specifically like to acknowledge Dr. Raju Rayavarapu, Joseph Miller, and Wei Zhuang for their collaborations and contributions to Chapter 3 of this dissertation. Raju showed me what true passion can achieve and without him this would not have been possible. Thank you to Dr. Jung Mi Park for always being willing to talk through experimental failures and successes and always reminding me to look at the bigger picture.

I would also like to recognize my collaborators Dr. Lily Huang, Dr. Sandra Miller, as well as my committee members Dr. Mondira Kundu, Dr. Joseph Opferman, and Dr. Sunny Wu for their work and input on a variety of experiments included in my dissertation. I would like to give special thanks to Dr. Fatima Rivas for being both my collaborator and my committee member, and always going above and beyond to give me advice and encouragement. 


\begin{abstract}
Mitochondria are vital organelles that coordinate cellular energy homeostasis and also have important roles in cell death. Therefore, the removal of damaged or excessive mitochondria through autophagy, a process called mitophagy, is critical for maintaining proper cellular function.

Much of the research elucidating the mechanism and regulation of autophagy was performed in yeast cells, so there is still a lot of mystery surrounding how mitophagy is promoted and regulated in mammals in general and in different tissues. The most well understood mechanism involved in mitophagy, is the PINK1-PARKN pathway. However, studies often rely on severe mitochondrial damage using CCCP that is considered non-physiological.
\end{abstract}

To find novel regulators of mitophagy in mammalian cells, we used a previously published screen for autophagy regulators to identify the MEKK3-MEK5-ERK5 pathway as a potential regulator of mitophagy in the absence of exogenous damage. Here, I provide evidence that genetic or pharmacological inhibition of the MEKK3-MEK5ERK5 pathway increases mitochondrial content by reducing lysosome-mediated degradation of mitochondria under basal conditions. I confirm that the MEKK3-MEK5ERK5 pathway is not required for non-selective bulk autophagy, damage-induced mitophagy, or restraint of mitochondrial biogenesis, confirming a selective role in basal mitochondrial degradation. Furthermore, inhibition of MEK5 or ERK5 in erythrocytes leads to defective mitochondrial elimination and impaired erythrocyte differentiation.

In addition to finding a novel mitophagy promoting pathway, we also worked to discover a novel mitophagy inducing compound. There are several different mechanisms for mitophagy induction, so there is considerable interest in the field in identifying novel chemical modulators that promote the efficient removal of mitochondria through different pathways. 


\section{TABLE OF CONTENTS}

CHAPTER 1. INTRODUCTION .....................................................................................1

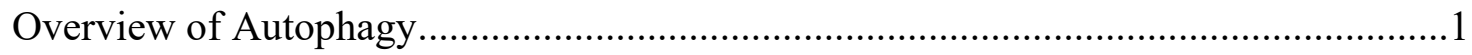

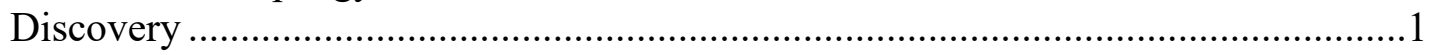

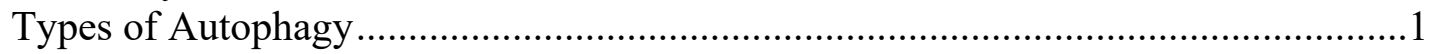

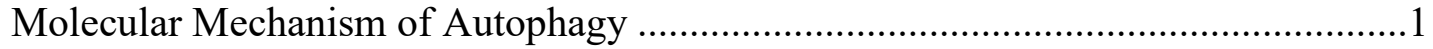

Selective versus Non-selective Autophagy .........................................................2

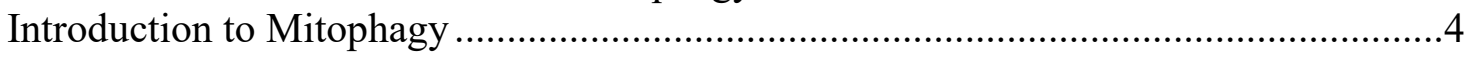

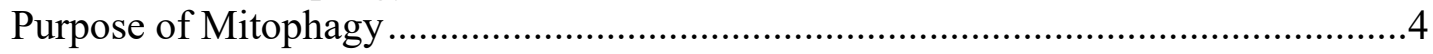

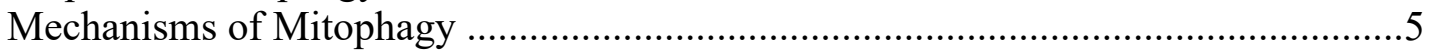

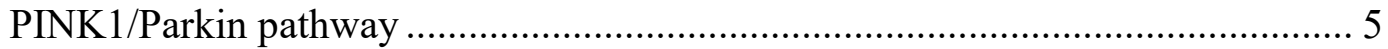

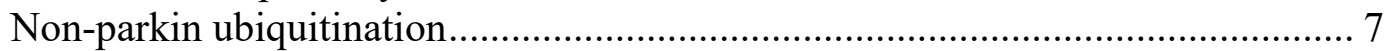

Mitochondrial proteins as mitophagy receptors............................................... 7

Mitophagy in Development and Disease .......................................................... 8

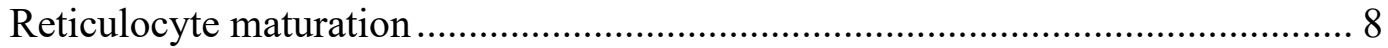

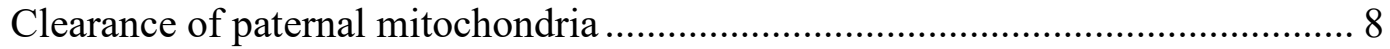

Stem cell maintenance .............................................................................. 8

Cardiovascular health............................................................................... 9

Neurodegenerative diseases .................................................................... 9

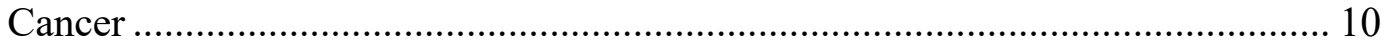

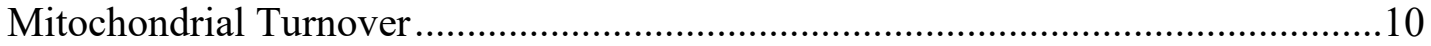

MEKK3-MEK5-ERK5 Kinase Cascade.................................................................. 11

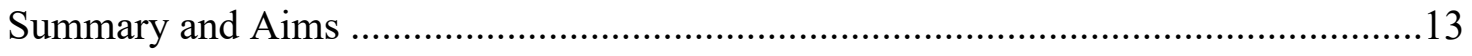

CHAPTER 2. MATERIALS AND METHODS ..................................................14

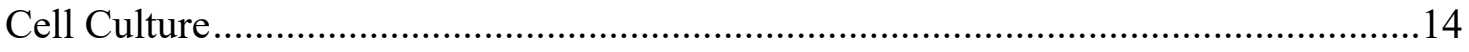

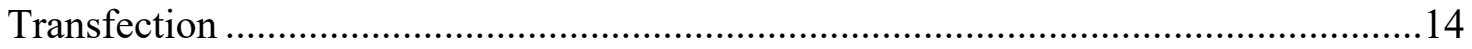

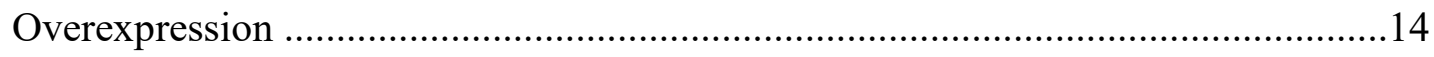

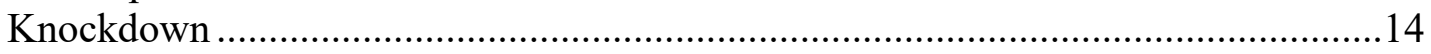

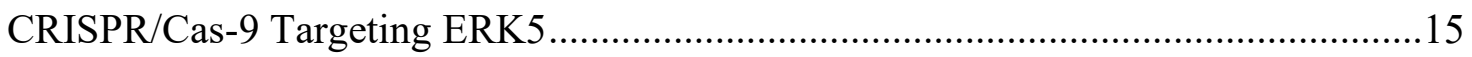

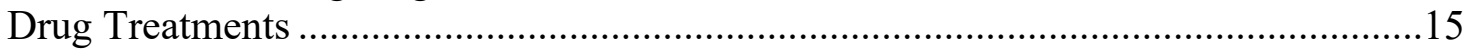

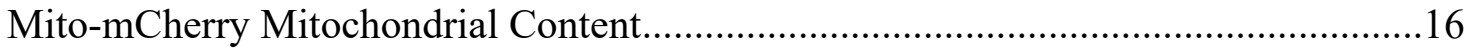

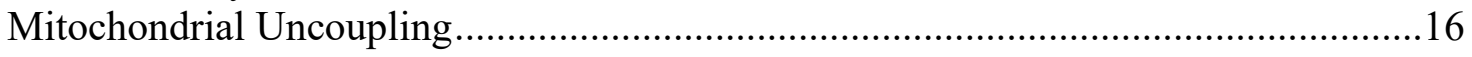

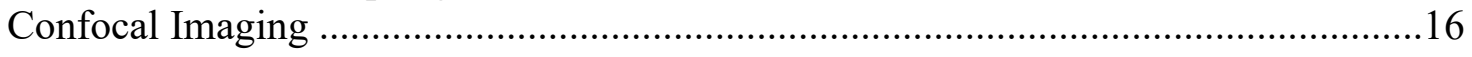

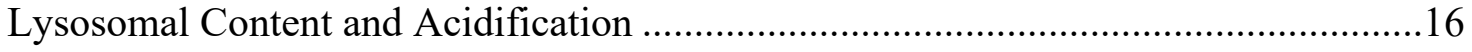

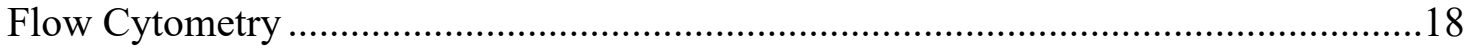

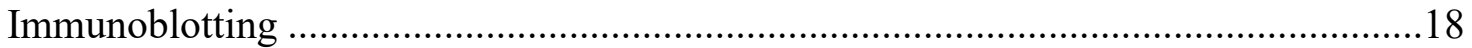

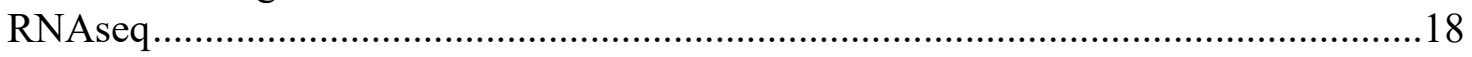

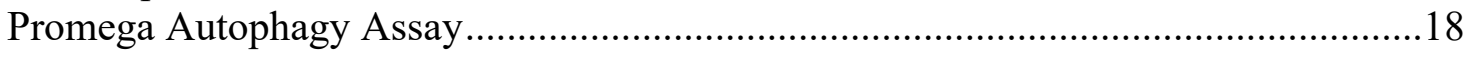

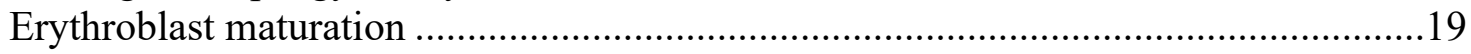

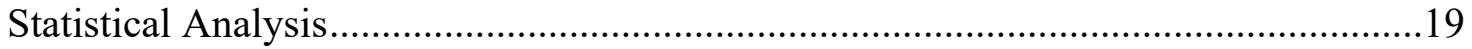




\section{CHAPTER 3. MEKK3-MEK5-ERK5 SIGNALING PROMOTES}

SQSTM1/p62 Constitutively Delivers Mitochondria to Lysosomes for Degradation in the Absence of Exogenous Damage

The MEKK3-MEK5-ERK5 Kinase Cascade Prevents Accumulation of Excess Mitochondria .26

The MEKK3-MEK5-ERK5 Pathway Is Required for Lysosomal Degradation of Mitochondria.

MEKK3-MEK5-ERK5 Pathway is Required for Differentiation and Mitophagy in Erythroid Progenitors

CHAPTER 4. IDENTIFYING JATROGOSSONE A AS A NOVEL

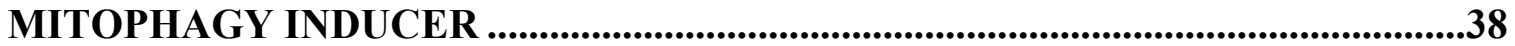

CHAPTER 5. DISCUSSION

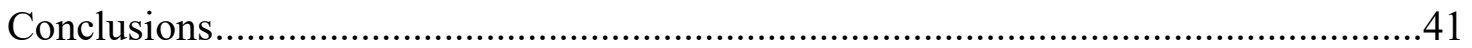

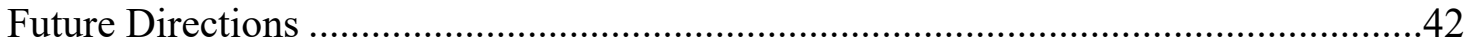

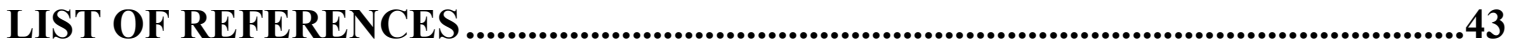

APPENDIX. BIX02188 AND BIX02189 KINOME PROFILING ............................54

VITA 


\section{LIST OF FIGURES}

Figure 1-1. Autophagy mechanism overview..........................................................

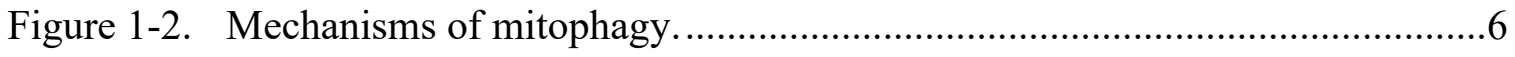

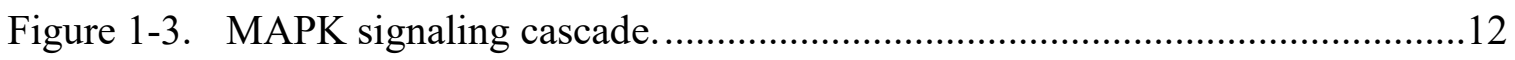

Figure 2-1. Mitochondrial targeted mKeima detects mitophagy events.......................17

Figure 3-1. Basal mitophagy is active in U2OS cells..............................................21

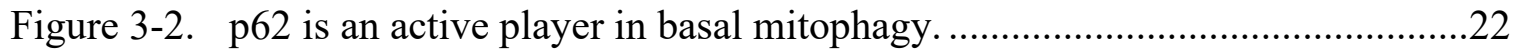

Figure 3-3 Identification of MEKK3/MEK5 as a novel pathway involved in

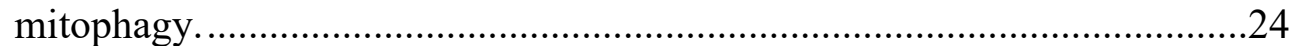

Figure 3-4. Confirmation that siRNA knockdown of MEKK3 and MEK5 increase mitochondrial content.....................................................................24

Figure 3-5. Pharmacological inhibition of MEK5 increase mitochondrial content........25

Figure 3-6. Genetic and pharmacological inhibition of downstream component ERK5 also increases mitochondrial content.

Figure 3-7. Pharmacological and genetic perturbation of the MEKK3/MEK5/ERK5 pathway cause accumulation of the SAR p62 ..........................................28

Figure 3-8. Perturbations of the MEKK3/MEK5/ERK5 pathway do not induce mitochondrial biogenesis.

Figure 3-9. The MEKK3/MEK5/ERK5 pathway does not promote autophagy nonselectively.

Figure 3-10. The MEKK3-MEK5-ERK5 pathway is required for lysosomal degradation of mitochondria.

Figure 3-11. The MEKK3-MEK5-ERK5 pathway is not required for CCCP-induced Parkin-mediated mitophagy.

Figure 3-12. MEK5 is required for differentiation and mitophagy in erythroid progenitors.

Figure 3-13. ERK5 is required for differentiation and mitophagy in erythroid progenitors.

Figure 3-14. Knockdown of MEK5 impairs erythroid differentiation. .37 
Figure 4-1. Treatment with Jatrophone but not Jatrogossone A causes severe alterations in mitochondrial morphology................................................39

Figure 4-2. Treatment with Jatrogossone A induces mitophagy. ...................................40 


\section{LIST OF ABBREVIATIONS}

$\begin{array}{ll}\mu M & \text { Micromolar } \\ \text { ARIH1 } & \text { Ariadne RBR E3 Ubiquitin Protein Ligase 1 } \\ \text { ATG } & \text { Atophagy related } \\ \text { ATP } & \text { Adenosine triphosphate } \\ \text { BMK1 } & \text { Big MAP Kinase 1 } \\ \text { BNIP3 } & \text { BCL2 Interacting Protein 3 } \\ \text { BNIP3L/NIX } & \text { BNIP3 like protein } \\ \text { CCCP } & \text { Carbonyl cyanide m-chlorophenyl hydrazone } \\ \text { CD71 } & \text { Cluster of Differentiation 71, atransferrin receptor } \\ \text { CMA } & \text { Chaperone mediated autophagy } \\ \text { DA } & \text { Dopaminergic neuron } \\ \text { DAPI } & \text { 4',6-diamidino-2-phenylindole } \\ \text { DNA } & \text { Deoxyribonucleic acid } \\ \text { Drp1 } & \text { Dynamin-related protein 1 } \\ \text { EDTA } & \text { Ethylenediaminetetraacetic acid } \\ \text { EPO } & \text { Erythropoietin } \\ \text { ERK } & \text { Extracellular-signal-regulated kinase } \\ \text { FBS } & \text { Fetal bovine serum } \\ \text { FUNDC1 } & \text { FUN14 Domain Containing 1 } \\ \text { FuSiOn } & \text { Functional signature ontology } \\ \text { GABARAP } & \text { GABA Type A Receptor-Associated Protein } \\ \text { GFP } & \text { Green fluorescent protein } \\ \text { IMM } & \text { Inner mitochondrial membrane } \\ \text { IPSCs } & \text { Induced pluripotent stem cells } \\ \text { JNK } & \text { c-Jun N-terminal kinases } \\ \text { KO } & \text { Knock out } \\ \text { LAMP1 } & \text { Lysosomal Associated Membrane Protein 1 } \\ \text { LC3 } & \text { Light chain 3 } \\ \text { LIR } & \text { LC3 interacting region } \\ \text { MAPK } & \text { Mitogen activated protein kinase } \\ \text { MEK } & \text { Mitogen/extracellular signal-regulated kinase } \\ \text { MFN1/2 } & \text { Mitofusin1/2 } \\ \text { MPP } & \text { Mitochondrial processing protease } \\ \text { mtDNA } & \text { Mitochondrial DNA } \\ \text { mTOR } & \text { Mechanistic target of rapamycin } \\ \text { MUL1 } & \text { Mitochondrial E3 Ubiquitin Protein Ligase 1 } \\ \text { NBR1 } & \text { Neighbor of BRCA1 gene 1 } \\ \text { NDP52 } & \text { Nuclear dot 52 } \\ \text { NLS } & \text { Nuclear localization signal } \\ \text { NRF1/2 } & \text { Nuclear Respiratory Factor 1 } \\ \text { OMM } & \text { Outer mitochondrial membrane } \\ \text { OPTN } & \text { Optineurin } \\ \text { OXPHOS } & \text { Oxidative phosphorylation } \\ & \end{array}$




$\begin{array}{ll}\text { PARL } & \text { Presenilins-associated rhomboid-like protein } \\ \text { PAS } & \text { Phagophore assembly site } \\ \text { PCR } & \text { Polymerase chain reaction } \\ \text { PD } & \text { Parkinson's Disease } \\ \text { PE } & \text { Phosphatidylethanolamine } \\ \text { PGC1 } \alpha & \text { Peroxisome proliferator activated receptor gamma coactivator 1 } \alpha \\ \text { PHB2 } & \text { Prohibitin 2 } \\ \text { PINK1 } & \text { PTEN-induced kinase 1 } \\ \text { RNA } & \text { Ribonucleic acid } \\ \text { RNAi } & \text { RNA interference } \\ \text { ROS } & \text { Reactive oxygen species } \\ \text { SAR } & \text { Selective autophagy receptor } \\ \text { SiRNA } & \text { small interfering RNA } \\ \text { shRNA } & \text { short hairpin RNA } \\ \text { SMURF1 } & \text { SMAD Specific E3 Ubiquitin Protein Ligase 1 } \\ \text { SQSTM1 } & \text { Sequestosome 1 } \\ \text { TAD } & \text { Transcriptional activation domain } \\ \text { TBK1 } & \text { TANK Binding Kinase 1 } \\ \text { TFAM } & \text { Transcription Factor A, Mitochondrial } \\ \text { TFB1/2M } & \text { Transcription Factor B1/2, Mitochondrial } \\ \text { TOMM } & \text { Translocase Of Outer Mitochondrial Membrane } \\ \text { Ub } & \text { Ubiquitin } \\ \text { ULK1/2 } & \text { Unc-51 Like Autophagy Activating Kinase 1 } \\ \text { USP } & \text { Ubiquitin Specific Peptidase } \\ \text { VCP } & \text { Valosin-containing protein } \\ \text { VDAC } & \text { Voltage-dependent anion-selective channel 1 } \\ \text { WT } & \text { Wild type } \\ \text { YFP } & \text { Yellow Fluorescent protein } \\ \end{array}$




\title{
CHAPTER 1. INTRODUCTION
}

\author{
Overview of Autophagy
}

\section{Discovery}

The field of autophagy research began when Christian de Duve discovered granules that were rich in hydrolytic enzymes while performing tissue fractionations on rat livers (De Duve, Pressman, Gianetto, Wattiaux, \& Appelmans, 1955; Glick, Barth, \& Macleod, 2010). He proposed that they be called lysosomes and hypothesized that they may be involved in localized intracellular digestion (De Duve et al., 1955). De Duve later termed the process of delivering cargo to the lysosome for digestion 'autophagy', meaning 'self-eating' in Greek (Glick et al., 2010).

Decades later, Yoshinori Ohsumi's laboratory used genetic screens on yeast (Saccharomyces cerevisiae), where he identified 15 genes that were required for autophagy (Tsukada \& Ohsumi, 1993). Subsequently, most of the breakthroughs in understanding how autophagy is regulated and executed have occurred in yeast as well. Now, over 40 autophagy related genes (ATGs) have been identified in yeast (Nakatogawa, Suzuki, Kamada, \& Ohsumi, 2009; Wen \& Klionsky, 2016). Though many of these ATGs are conserved in other orders, including mammals, differences in how autophagy is regulated are an active research topic (Glick et al., 2010).

\section{Types of Autophagy}

Autophagy can be divided into either chaperone mediated autophagy (CMA), microautophagy or macroautophagy based on how the substrates are targeted and the mechanism by which intracellular materials are delivered into the lysosome for degradation (Khandia et al., 2019). Although these pathways are mechanistically distinct, they all carry out degradation via the lysosome (Khandia et al., 2019). In CMA, a chaperone protein complex targets proteins for degradation and translocated them across the lysosomal membrane (Cuervo \& Wong, 2014). Microautophagy occurs when the lysosome, itself, invaginates and engulfs small sections of the cytoplasm (Mijaljica, Prescott, \& Devenish, 2011). Macroautophagy (here after referred to as autophagy) occurs when a portion of cytoplasm is sequestered by a forming autophagosome before fusing with a lysosome (Mizushima, 2007).

\section{Molecular Mechanism of Autophagy}

Autophagy can be induced by several different signaling pathways and by many different stimuli such as nutrient depravation (Hosokawa et al., 2009), oxidative stress (Filomeni, De Zio, \& Cecconi, 2015), hypoxia (L. Liu et al., 2012), and infection 
(Khandia et al., 2019). In the presence of nutrients and cytokines, mechanistic target of rapamycin (mTOR) blocks apoptosis and stimulates cell growth (Hosokawa et al., 2009). On the other hand, starvation and stress inhibit mTOR which leads to autophagy initiation (Hosokawa et al., 2009).

The first protein complex involved in autophagy induction is the autophagy initiation complex, composed of ULK1, ATG13, FIP200, and ATG101 (Dikic \& Elazar, 2018). When autophagy is initiated, ATGs are recruited to a specific location within the cell called the phagophore assembly site (PAS) where the phagophore membrane is nucleated by the PI3KC3 complex I (composed of VSP34, Beclin1, ATG14, AMBRA1, and p115 (Dikic \& Elazar, 2018). After nucleation, the phagophore membrane is elongated and decorated with ATG8 family proteins such as LC3. First, free LC3 (LC3-I) must be conjugated to the membrane bound lipid phosphatidylethanolamine (PE) to become LC3-II (Dikic \& Elazar, 2018). Conjugation of ATG8s to PE promotes phagophore expansion and possibly has a role in sealing the phagophore into a complete vesicle called an autophagosome (T. N. Nguyen et al., 2016). Autophagosomes then fuse with highly acidic lysosomes that contain hydrolases which degrade any cargo inside (Figure 1-1) (Dikic, 2017; Mizushima, 2007).

\section{Selective versus Non-selective Autophagy}

There are two major types of degradation within autophagy: selective and nonselective. In non-selective autophagy starvation triggers cytoplasmic material to be engulfed by the autophagosome in a non-specific manner and the contents are all degraded by the lysosome (Mizushima, 2007). Whereas in selective autophagy certain signals can induce a highly selective targeting of specific cellular structures, such as damaged mitochondria (mitophagy), invading bacteria (xenophagy), misfolded/aggregated proteins (aggrephagy) as well as many other substrates (Zaffagnini $\&$ Martens, 2016). Selective autophagy often requires cargo to be labelled with a tag for degradation (most notably chains of ubiquitin) (Stolz, Ernst, \& Dikic, 2014). These degradation tags are then recognized by autophagy receptors that link the cargo to the autophagic membrane via their LC3-interacting region (LIR); though some cargo receptors are able to bind cargo directly (Kirkin, 2020; Pankiv et al., 2007). There are a growing number of selective autophagy receptors (SARs) including NBR1, OPTN, NIX and SQSTM1/p62 (Zaffagnini \& Martens, 2016). The most well characterized example of a SAR is p62/SQSTM1, an adaptor protein that participates in several types of selective autophagy including mitophagy and aggrephagy.(Glick et al., 2010). Although SARs themselves are not highly conserved, their modes of action and the signaling cascades that activate and regulate them are (Farre \& Subramani, 2016).

Furthermore, there are two forms of selective autophagy: constitutive and induced (Mizushima, 2005). Constitutive autophagy (also known as basal autophagy) has not been well studied, whereas induced autophagy has been much more extensively studied. Induced autophagy can be triggered by cellular damage, dysfunctional organelles, as well 

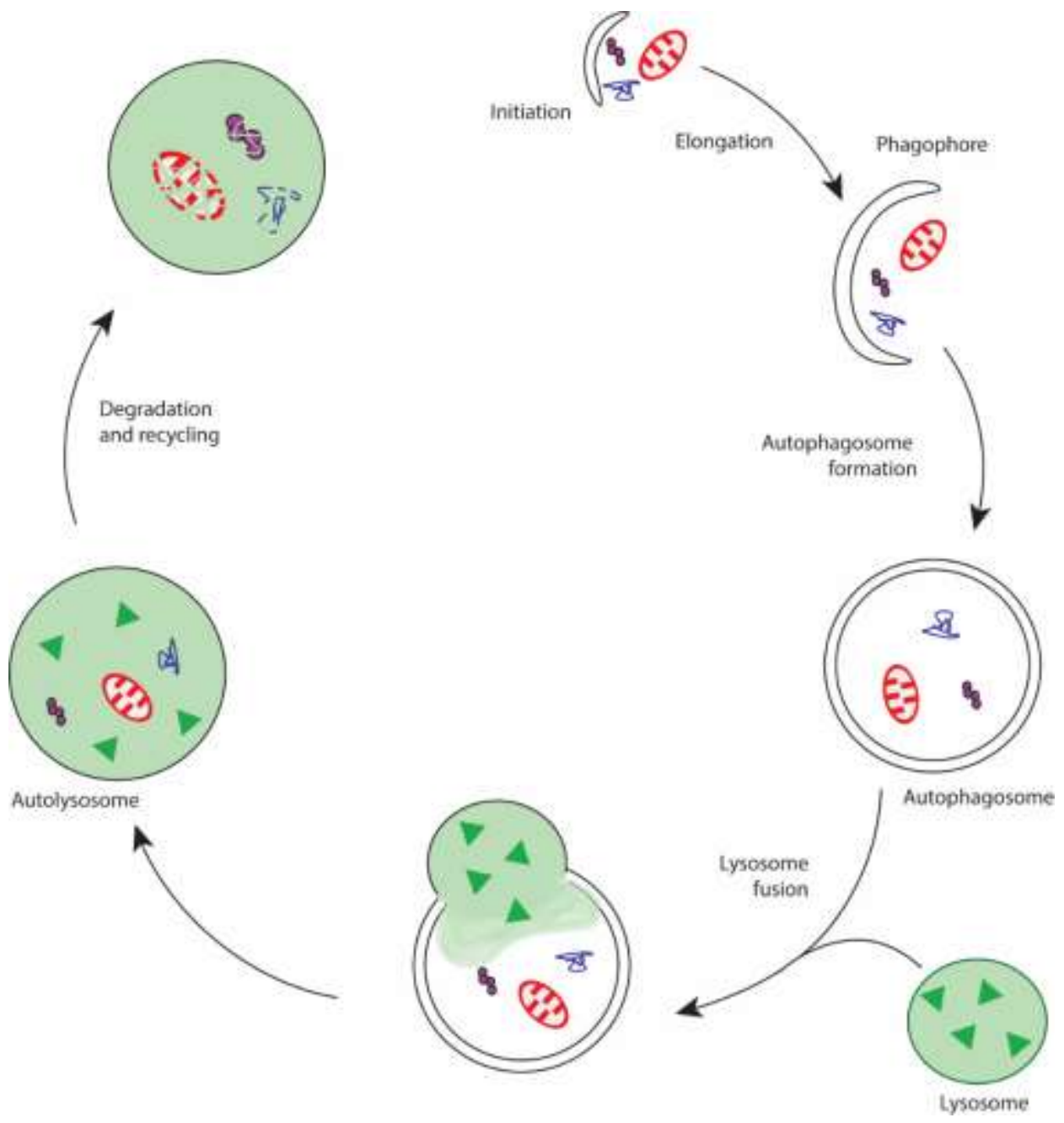

Figure 1-1. Autophagy mechanism overview.

An expanding membrane structure (phagophore) forms a double-membrane vesicle (autophagosome) that sequesters a portion of the cytoplasm. The autophagosome then fuses with the lysosome, exposing its inner components to the hydrolases and highly acidic environment in the lysosomal interior. The inner membrane of the autophagosome is degraded along with the cargo inside. The resulting macromolecules are then released into the cytosol for recycling.

Source: Mizushima N. Autophagy: process and function. Genes Dev. 2007;21(22):286173. 
as developmental programs (Glick et al., 2010). Basal autophagy is thought to have a housekeeping role and acts to turn over cytosolic components such as mitochondria (Mizushima, 2005, 2007).

\section{Introduction to Mitophagy}

The degradation of mitochondria through autophagy is known as mitophagy. Mitophagy was first observed using electron microscopy, but it took almost 40 years for researchers to make any breakthroughs in understanding why and how mitochondria were being degraded (Ashford \& Porter, 1962; Clark, 1957). The first sign that individual mitochondrion could be selected for degradation came from J. J. Lemasters' lab when they reported that only depolarized mitochondria were sequestered by autophagosomes (Elmore, Qian, Grissom, \& Lemasters, 2001). Early insights into the mechanistic basis of mitochondrial degradation arose from studies in yeast, when the protein ATG32 was identified as a key mitophagy receptor (Kanki, Wang, Cao, Baba, \& Klionsky, 2009; Okamoto, Kondo-Okamoto, \& Ohsumi, 2009). Around the same time, insights into mammalian mitophagy regulation were also being made. BNIP3L/NIX was discovered to regulate programmed mitochondrial clearance during reticulocyte maturation (Schweers et al., 2007). Parkin was shown to have a critical role in targeting depolarized mitochondria to be degraded (Narendra, Tanaka, Suen, \& Youle, 2008). Several other mitophagy receptors and signaling mechanisms have been discovered since, however, there is still much left to understand about how mitophagy signaling is regulated in vivo.

\section{Purpose of Mitophagy}

Maintaining a population of healthy mitochondria is vital for proper development and is necessary to preserve tissue and organ function in response to stress conditions (Pickles, Vigie, \& Youle, 2018). Mitochondria are well known for their importance in metabolism and energy production, but they are also a major source of reactive oxygen species (ROS), cytosolic DNA, and pro-apoptotic proteins (Kausar, Wang, \& Cui, 2018; McArthur et al., 2018; Murphy, 2009; West et al., 2015). Therefore, it is important to maintain sufficient quantities of healthy mitochondria to meet current metabolic demand, but dangerous to carry an excessive surplus of these organelles.

Damaged or dysfunctional mitochondria tend to generate higher levels of ROS and cytosolic DNA compared to healthy mitochondria (Kausar et al., 2018; Murphy, 2009; Sliter et al., 2018). Exposure to high levels of ROS can cause DNA mutations in both nuclear and mitochondrial DNA and can induce protein misfolding throughout the cell (Kausar et al., 2018; Murphy, 2009). Mutations in mitochondrial DNA further decrease the health of the mitochondrial population and promote additional ROS production (Hahn \& Zuryn, 2019; Mattiazzi et al., 2004; Nissanka \& Moraes, 2018). There are other situations in which mitochondrial content changes even in the absence of damage, such as during differentiation of highly specialized cell types or induction of a metabolic shift towards a glycolytic phenotype or an oxidative phosphorylation 
phenotype (Esteban-Martinez \& Boya, 2018; Esteban-Martinez et al., 2017; Hu et al., 2016; Palikaras, Lionaki, \& Tavernarakis, 2018) Thus to maintain homeostasis, the cell has evolved complex systems for the quality control of mitochondria (Ding \& Yin, 2012). These systems are designed to balance the elimination of dysfunctional or superfluous mitochondria with mitochondrial biogenesis (Pickles et al., 2018)..

\section{Mechanisms of Mitophagy}

In mammals, mitophagy is induced by a variety of cellular stimuli, including cellular differentiation; hypoxic conditions; mitochondrial damage; and paternal mitochondrial elimination (Pickles et al., 2018). Interestingly, several different molecules and mechanisms are utilized in mitophagy depending on the needs of the cell (Figure 1-2).

\section{PINK1/Parkin pathway}

The most well studied mechanism for targeting damaged mitochondria for elimination in metazoans, such as $\mathrm{C}$. elegans and mammals, usually involves two proteins: PINK1, a kinase that constitutively localizes to the mitochondria and Parkin, a cytosolic E3 ubiquitin ligase. When mitochondria are healthy and have a strong membrane potential, PINK1 is imported into the mitochondria through the outer mitochondrial membrane and then the inner mitochondrial membrane (Jin et al., 2010). While passing through the inner membrane, PINK1 is cleaved by the proteases in the mitochondrial matrix (MPP and PARL) (Jin et al., 2010). The cleavage product is then released into the cytoplasm and degraded (Pickles et al., 2018). However, mitochondria with low membrane potential cannot successfully import PINK1 past the mitochondrial inner membrane, preventing PINK1 from being cleaved (Jin et al., 2010). This causes PINK1 to accumulate on the surface of the mitochondria. PINK1 can then form dimers and auto-phosphorylate (Okatsu et al., 2013). PINK1 kinase activity is necessary and sufficient for the recruitment of Parkin to mitochondria (Lazarou, Jin, Kane, \& Youle, 2012). In addition to auto-phosphorylation, PINK1 also phosphorylates Parkin to increase its E3-ligase activity, allowing it to ubiquitinate substrates (Lazarou et al., 2012; ShibaFukushima et al., 2012). PINK1 can then phosphorylate the ubiquitin at Ser65, which causes a structural change in ubiquitin and makes it resistant to deubiquitinating enzymes (Shiba-Fukushima et al., 2012). Polyubiquitin chains act as an "eat me" signal for damages organelles, so deubiquitinating enzymes, such as USP15 and USP30, inhibit mitophagy by removing the ubiquitin chains from the mitochondrial surface (Palikaras et al., 2018). Though this pathway has been very well characterized, most studies depend on ectopic Parkin overexpression and mitochondrial depolarization with CCCP or other pharmacological agents (Drake, Springer, Poole, Kim, \& Macleod, 2017; Lazarou et al., 2015; Narendra et al., 2008). Additionally, endogenous Parkin is poorly expressed in several mammalian tissues and cell lines, so more work is needed to elucidate how mitophagy is regulated in those tissues (Huynh, Dy, Nguyen, Kiehl, \& Pulst, 2001; McWilliams et al., 2018). 


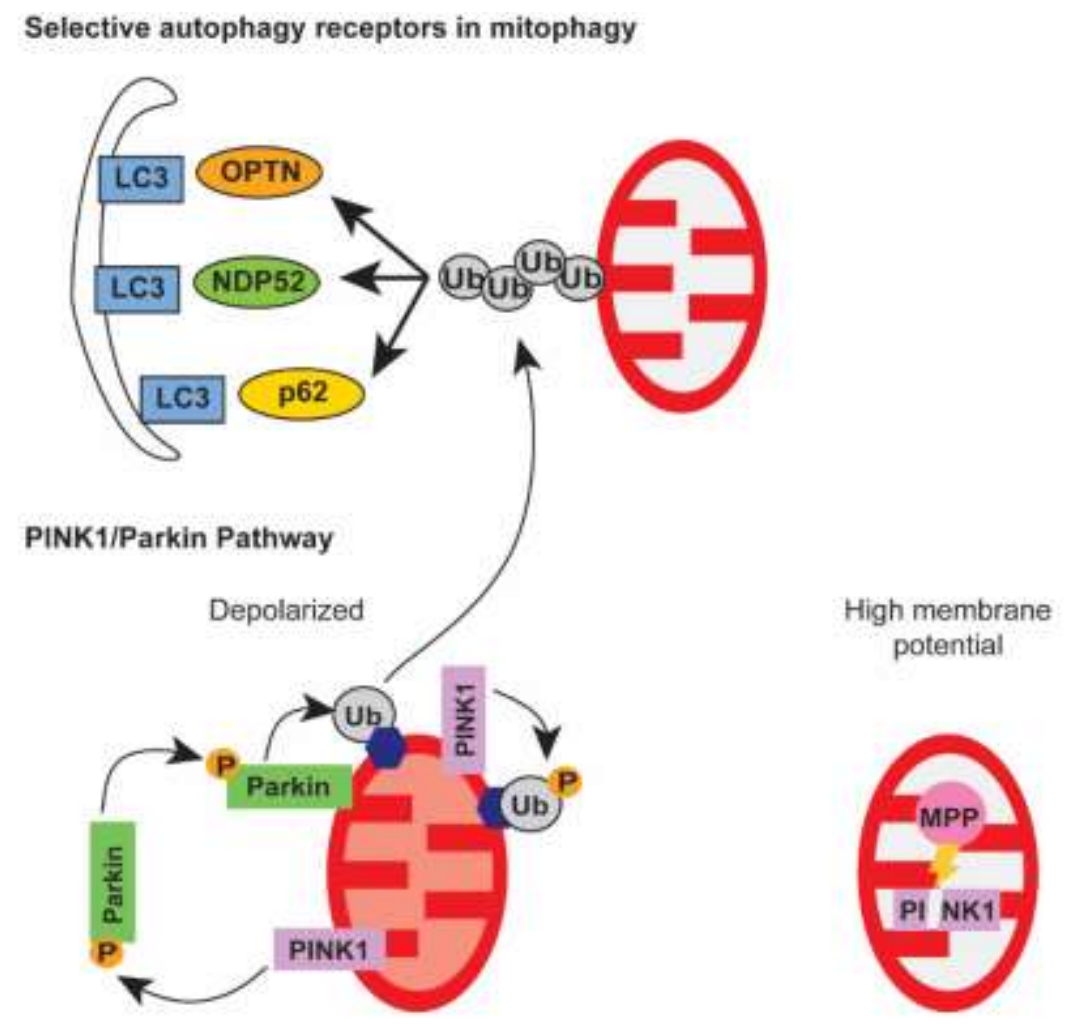

Mitochondrial proteins as mitophagy receptors

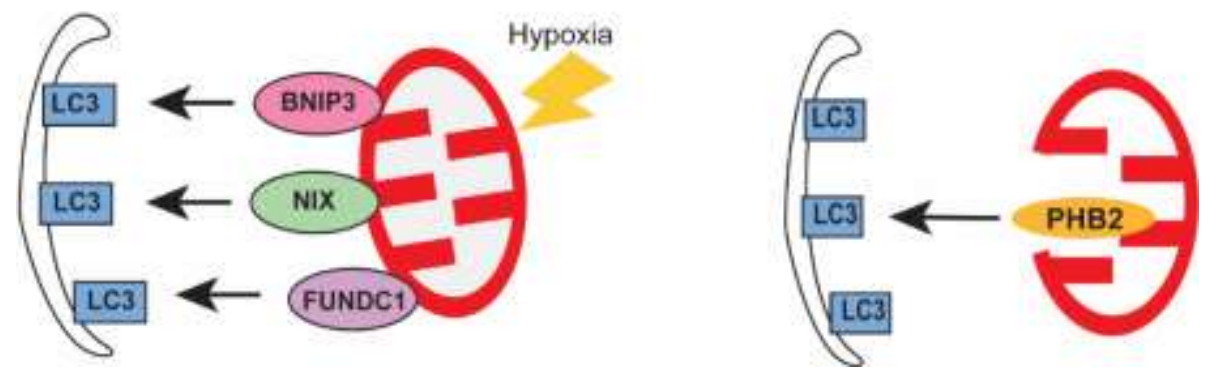

Figure 1-2. Mechanisms of mitophagy.

Polyubiquitinated proteins are recognized by SARs, including p62, OPTN, and NDP52. SARs recognize and bind LC3 to promote autophagosome formation. PINK1 accumulates on the surface of mitochondria with low membrane potential. PINK1 recruits Parkin by phosphorylating both Parkin and ubiquitin. Parkin then polyubiquitinates OMM proteins such as VDAC1 and MFN1/2. Receptor-mediated mitophagy also utilizes various OMM proteins such as BNIP3, NIX, and FUNDC1. In addition, PHB2 can also act as an inner mitochondrial membrane receptor in cases where the mitochondrial membranes rupture and PHB2 is exposed to the cytosol where it can bind LC3.

Source: Palikaras K, Lionaki E, Tavernarakis N. Mechanisms of mitophagy in cellular homeostasis, physiology and pathology. Nat Cell Biol. 2018;20(9):1013-22. 


\section{Non-parkin ubiquitination}

In addition to Parkin, there are several other E3 ligases, such as SMURF1, MUL1 and ARIH1, that also regulate mitophagy through ubiquitination (Palikaras et al., 2018). They are recruited to the mitochondrial outer membrane where they create ubiquitin chains, allowing autophagy adaptor proteins, including optineurin (OPTN), nuclear dot protein 52 (NDP52), NBR1 and p62, to bind (Yoo \& Jung, 2018). Core autophagy proteins are then recruited to mediate phagophore biogenesis and autophagosomal membrane expansion (Palikaras et al., 2018). The serine/threonine-protein kinase TBK1 (TANK binding kinase 1) can phosphorylate mitophagy adaptors like OPTN, NDP52 and p62 to enhances their ability to bind to ubiquitin chains, promoting mitochondrial removal (Richter et al., 2016). Several studies have shown that p62 is not required for mitophagy to take place (Narendra, Kane, Hauser, Fearnley, \& Youle, 2010). Therefore, the importance and function of p62 in mitophagy is controversial and requires further investigation into cell type- and tissue-specific contexts.

\section{Mitochondrial proteins as mitophagy receptors}

In addition to ubiquitin dependent mitophagy, some outer mitochondrial membrane proteins like NIX, BNIP3, and FUNDC1 also act as mitophagy receptors, targeting mitochondria directly to autophagosomes for degradation. NIX has been reported to interact with ATG8 family members GABARAP and LC3 through this LIR domain, although the role of this interaction has been contested (Novak et al., 2010). BNIP3 is about $50 \%$ homologous to Nix and also acts as a mitophagy receptor (Pickles et al., 2018). Nix and BNIP3 are both reported to have roles in hypoxia induced mitophagy, and protein levels for both are transcriptionally increased under hypoxic conditions (Palikaras et al., 2018). BNIP3 has additional roles in promoting the fission of damaged mitochondria by recruiting mitochondrial fission protein Drp1 to the outer mitochondrial membrane (Y. Lee, Lee, Hanna, \& Gustafsson, 2011). There is also evidence of cross talk between PINK1/Parkin mitophagy and both BNIP3 and Nix mediated mitophagy. BNIP3 increases PINK1 stability on the mitochondrial surface by inhibiting its cleavage, and Nix is a Parkin ubiquitination substrate (Gao et al., 2015; T. Zhang et al., 2016). FUNDC1 is another outer mitochondrial membrane protein that promotes mitophagy during hypoxia (Yoo \& Jung, 2018). Under normoxic conditions, FUNDC1's LIR motif is kept inactive by phosphorylation at Ser13 and Tyr18 (Chen et al., 2014; L. Liu et al., 2012). Under hypoxic conditions, FUNDC1 is dephosphorylated at Ser13 by PGAM5 and phosphorylated by ULK1 at Ser17 which allows the LIR motif to interact with LC3 (Chen et al., 2014). Proteins in the inner mitochondrial membrane can also act as mitophagy receptors. Prohibitin2 (PHB2) is a protein in the inner mitochondrial membrane that has been shown to mediate Parkin-dependent mitophagy (Wei, Chiang, Sumpter, Mishra, \& Levine, 2017). When the mitochondrial membrane is depolarized and the outer membrane ruptures, PHB2 becomes exposed to the cytoplasm where it can associate with LC3 and p62 to generate a phagophore (Wei et al., 2017; Xiao, Zhou, Lu, Zhou, \& Cai, 2018). 


\section{Mitophagy in Development and Disease}

\section{Reticulocyte maturation}

In addition to its role in removing damaged mitochondria, mitophagy must also remove perfect healthy mitochondria during key stage in development (Gustafsson \& Dorn, 2019). In most mammals, mitochondria are absent from mature red blood cells (Mortensen et al., 2010). During late stages of hematopoietic stem cell differentiation, reticulocytes (enucleated immature red blood cells) must lose their mitochondria to become mature erythrocytes (Mortensen et al., 2010). Nix protein levels are increased on the surface of the mitochondria during the transition from reticulocyte to erythrocyte (Aerbajinai, Giattina, Lee, Raffeld, \& Miller, 2003), and mature red blood cells from NIX knockout mice have increased mitochondrial content (Schweers et al., 2007). Interestingly, mitochondrial clearance in reticulocytes does not occur through the canonical ATG5/ATG7 autophagy pathway (Honda et al., 2014). However, it has been shown that mitochondrial clearance is dependent on Ulk1, as murine reticulocytes lacking Ulk1 have an impaired ability to clear mitochondria (Kundu et al., 2008).

\section{Clearance of paternal mitochondria}

In most animals, from flies and worms to mice and humans, mitochondrial DNA (mtDNA) is only inherited maternally. It was unknown whether paternal mtDNA was lost due to dilution, as there is a relatively small number of mitochondria in sperm cells or if paternal mitochondria were actively targeted for degradation. Deep sequencing has found no residual amounts of paternal mtDNA in most cases, indicating that paternal mitochondria are likely actively eliminated. Mice engineered to possess a mixture of two different sets of mtDNA had abnormal metabolism, decreased cognitive function, and increased stress, providing evidence for why evolution has favored single parent inheritance (Sharpley et al., 2012). There is conflicting evidence for whether autophagy mediates paternal mtDNA elimination in mammals (Luo et al., 2013; Sutovsky et al., 2000). In fertilized oocytes, p62, GABARAP and VCP (shown to be essential for PINK1/Parkin mitophagy (Kim et al., 2013)) quickly aggregate around sperm mitochondria, and inhibition of either p62 or VCP delays the removal of sperm mitochondria (Song, Yi, Sutovsky, Meyers, \& Sutovsky, 2016). Knocking down p62, PINK1, or the mitochondrial fission protein, Fis1, in mouse embryos resulted in impaired paternal mitochondrial removal (Rojansky, Cha, \& Chan, 2016). When mitochondrial E3 ligases, Parkin and Mul1, were both knocked down embryos also retained paternal mitochondria (Rojansky et al., 2016). All together, these studies indicate that autophagy and, more specifically, mitophagy-related proteins have an important role in paternal mtDNA elimination.

\section{Stem cell maintenance}

Interestingly, the metabolic status of embryonic stem cells has a key role in maintaining stemness as well as differentiation (Gustafsson \& Dorn, 2019). Generating pluripotent stem cell using somatic cell reprogramming involves an initial increase in 
mitochondrial content followed by a decrease that relies upon Nix-dependent mitophagy (Palikaras et al., 2018). Additionally, loss of PINK1-dependent mitophagy resulted in dramatically decreased efficiency of induced pluripotent stem cell reprogramming and altered metabolism (Vazquez-Martin et al., 2016). Induced pluripotent stem cells (iPSCs) are highly glycolytic and contain few mitochondria which are small and rounded with condensed cristae and the low membrane potential (Palikaras et al., 2018). Conversely, differentiation induces mitochondria to become long and tubular in shape, mitochondrial mass increases, and cellular metabolism switches to OXPHOS (Vazquez-Martin et al., 2016). Mitophagy-deficient iPSCs have attenuated glycolysis and contain a mixture of mature and immature mitochondria (Vazquez-Martin et al., 2016). Mitophagy-deficient iPSC colonies also tend to spontaneously differentiate and form heterogeneous populations of cells (Vazquez-Martin et al., 2016).

\section{Cardiovascular health}

Mitophagy is important for both cardio myocyte maturation as well as mitochondrial maintenance in adult heart cells. Fetal mitochondria primarily use glucose for ATP production, while adult heart cells have far greater metabolic demands, so they utilize fatty acids for ATP production (Palikaras et al., 2018). During the maturation process, PINK1, Parkin, and mitochondrial fusion protein MFN2 act together to eliminate fetal mitochondria (Palikaras et al., 2018). In fact, cardiac specific deletion of Parkin during the metabolic remodeling process is lethal due to disruption of mitochondrial maturation and retention of fetal mitochondria in myocytes (Gustafsson \& Dorn, 2019). Mitophagy continues to play an important role in the maintenance of the adult cardiovascular system. The human heart is highly metabolically active organ (Gustafsson \& Dorn, 2019). Mitochondrial function and impaired mitophagy strongly influence cardiac function and have been implicated in several cardiomyopathies (Palikaras et al., 2018). PINK1 protein levels are significantly reduced in end-stage heart failure in humans (Billia et al., 2011). Cardiomyocytes of PINK1 null mice have altered mitochondrial morphology, increased mitochondrial content, and elevated ROS levels (Billia et al., 2011). PINK1 null mice also exhibit pathological cardiac hypertrophy and ventricular dysfunction (Billia et al., 2011). Additionally, myocytes activate mitophagy after ischemia to clear damaged mitochondria before they cause harm to the cell (Hamacher-Brady et al., 2007). Studies have also shown that impaired mitophagy in cardiomyoctes leads to increased susceptibility to stress and heart failure (Ikeda et al., 2015; Kubli et al., 2013).

\section{Neurodegenerative diseases}

Parkinson's disease (PD) is a neurodegenerative disorder characterized motor symptoms such as tremors. PD is caused by the loss of dopaminergic (DA) neurons in a region of the brain called the substantia nigra, (Airavaara et al., 2020). Mitochondrial dysfunction has been implicated in studies of sporadic, familial, and pharmacologically induced forms of PD (Gao et al., 2017). Additionally, loss-of-function mutations in PINK1 and Parkin have been identified in hereditary PD, and mutation in PINK1/Parkin homologues cause Parkinson's like symptoms in flies (Greene et al., 2003; Y. Yang et al., 
2006). On the other hand, Parkin and PINK1 perturbations do not phenocopy clinical PD in mice (Blesa \& Przedborski, 2014). However, POLG mutator mice that also lack Parkin do show a decrease in DA neurons (Pickrell et al., 2015). POLG mutator mice accumulate dysfunctional mitochondria due to proofreading deficiency in a DNA polymerase which causes an increase in mtDNA mutations (Trifunovic et al., 2004). More work needs to be done to elucidate how impaired PINK1 and Parkin signaling contribute to the development of PD and if they have roles maintaining mitochondrial health outside of mitophagy.

\section{Cancer}

The role of mitophagy in cancer progression is still controversial and appears to depend on cancer type. Studies have shown a correlation between impaired Parkin activity in many kinds of cancer such as glioblastoma, breast cancer, and ovarian cancer (Denison et al., 2003; Shah et al., 2012; Veeriah et al., 2010). Parkin knockout mice have been shown to develop hepatocellular carcinoma and have increased vulnerability to gamma irradiation induced tumorigenesis (Fujiwara et al., 2008; C. Zhang et al., 2011). However, whether Parkin's tumor suppression properties are due to Parkin's role in mitophagy has yet to be shown. It has been proposed that by eliminating dysfunctional mitochondria, mitophagy may be reducing oxidative damage and suppressing initiation of cancer (Gustafsson \& Dorn, 2019).

\section{Mitochondrial Turnover}

Mitochondrial turnover refers to the process of old, damaged, or dysfunction mitochondria or subsets of mitochondrial proteins being eliminated and then replaced with new mitochondria. Mitophagy is only one of several quality control mechanisms for mitochondria. Other methods include proteases in the mitochondrial matrix and inner membrane space, proteasome-dependent degradation of mitochondrial proteins, and the mitochondrial unfolded protein response (Pickles et al., 2018). In order to balance mitochondrial elimination, mitochondrial biogenesis is needed. Mitochondria have their own separate genomes that must act in coordination with the nuclear genome in order to produce new mitochondria. PGC1 $1 \alpha$, considered to be the master mitochondrial biogenesis transcription factor, stimulates NRF1 and NRF2 gene expression in the nucleus (Scarpulla, 2011). NRF1 and NRF2 activate the transcription of nuclear encoded mitochondrial proteins including the protein import machinery, the co-factors for respiratory chain complex assembly, and the mitochondrial DNA transcription and translation regulatory factors, including mitochondrial transcription factor A (Tfam) (Gleyzer, Vercauteren, \& Scarpulla, 2005). Tfam is required for mitochondrial gene transcription and for regulating mtDNA copy number (Scarpulla, 2011). 


\section{MEKK3-MEK5-ERK5 Kinase Cascade}

MEK5 and ERK 5 kinases are part of a family called the mitogen-activated protein kinases (MAPKs) are which are a group of highly conserved enzymes that are expressed in all eukaryotic cells and mediate several different intracellular processes such as growth, differentiation, and apoptosis (Drew, Burow, \& Beckman, 2012). MAPK pathways are three-tiered kinase cascades (Figure 1-3). Kinases at the top of the cascade are referred to as the MAPK kinase kinase (MAPKKK). MAPKKKs are often stimulated by extracellular stimuli like growth factors (Nithianandarajah-Jones, Wilm, Goldring, Muller, \& Cross, 2012). The MAPKKK then phosphorylates and activates the downstream kinase, the MAPK kinase (MAPKK) on specific serine and threonine residues (Nithianandarajah-Jones et al., 2012). The MAPKK then activates the final member of the cascade, the MAPK, by phosphorylating the threonine and tyrosine residues of the T-X-Y motif which is common among all MAPKs (NithianandarajahJones et al., 2012). Once the final MAPK has been activated, it can then phosphorylate and modify the behavior of its downstream effector molecules. In mammals there are distinct subfamilies of MAPKs such as the ERK 1/2 cascade, the ERK5 cascade, the JNK $1 / 2 / 3$ cascade, and the p38 proteins, (Drew et al., 2012). ERK5 is the most recently identified MAPK and regulates a variety of cellular processes including cell survival, proliferation, migration, and angiogenesis (Drew et al., 2012).

ERK5 was discovered in 1995 by two separate groups. J. Dixon's group first identified ERK5's upstream kinase, MEK5, and used a yeast two-hybrid screen to discover ERK5 (G. Zhou, Bao, \& Dixon, 1995). J. Han's group used PCR to identify a novel MAPK gene in a human placenta cDNA library, which they named Big MAPK 1 (BMK1) as it is much larger in size compared to ERK1/2 (J. D. Lee, Ulevitch, \& Han, 1995). ERK 5 and BMK1 were found to be the same protein. Together the groups showed that ERK5 is abundantly expressed in the heart, skeletal muscle, placenta, lungs and kidneys (J. D. Lee et al., 1995; G. Zhou et al., 1995). It was later shown that ERK5 is ubiquitously expressed in a variety of tissues and cell lines and is present in both nuclear and cytoplasmic compartments.(Nithianandarajah-Jones et al., 2012)

Compared to the other MAPKs, ERK5 has an elongated C-terminal tail that is over 400 amino acids long, making it over twice the molecular weight of many other MAPKs (G. Zhou et al., 1995). Within the C-terminal domain there is a nuclear localization signal (NLS) domain which allows ERK5 to translocate to the nucleus two proline-rich (PR) domains which are thought to be binding sites for SH3-domaincontaining proteins, a MEF2-interacting region, and transcriptional activation domain (TAD) (Nithianandarajah-Jones et al., 2012). The TAD can be autophosphorylated allowing ERK5 to directly regulate gene transcription, which is a unique ability compared to conventional MAPKs (Nishimoto \& Nishida, 2006).

In ERK5's inactive and unphosphorylated state, there is an interaction between the $\mathrm{N}$ and $\mathrm{C}$ terminals that holds ERK5 in a folded conformation (Nithianandarajah-Jones et al., 2012). This interaction prevents NLS signaling and may also signal for nuclear 


\section{MEKK2/3}

MEK1/2

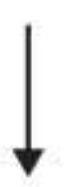

MAPK

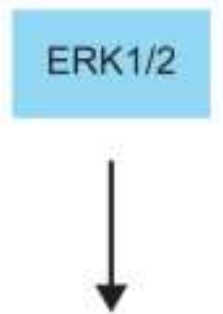

p90, ELK-1 STAT $1 / 3$

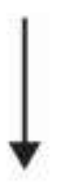

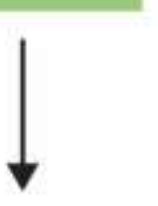

MEK5

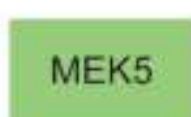

\section{ERK5}

p38

$\mathrm{MEK} 3 / 6$
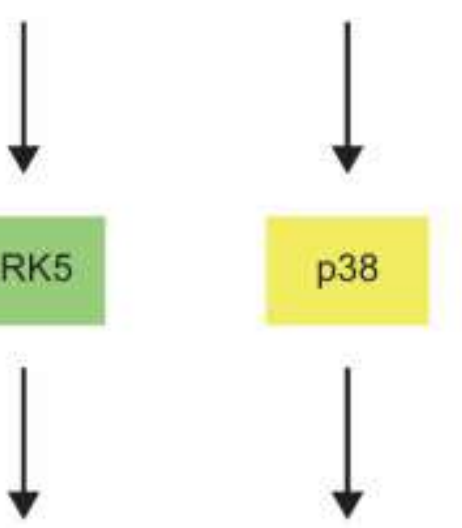

MEF2C, SGK, CX43
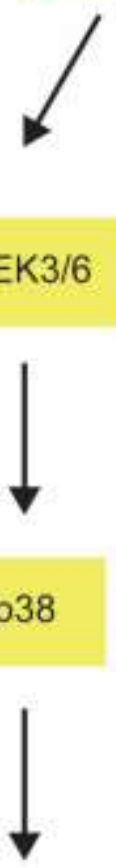
MEF2C, Myc

\section{MEKK $1 / 2 / 3 / 4$}

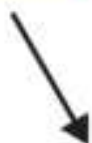

MEK4/7

ATF-2, Hsp27

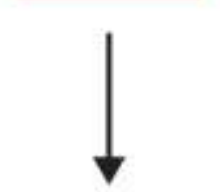

JNK $1 / 2 / 3$

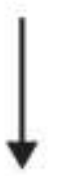

C-Jun, ELK-1, p53

Figure 1-3. MAPK signaling cascade.

Mammalian cells have four MAPK signaling cascades: ERK1/2, ERK5, p38 MAPKs and JNK $1 / 2 / 3$, that are organized into a system with three tiers known as a MAPK module. Stimuli such as cytokines, growth factors, and environmental stresses activate various MAPK cascades. First, the apical MAPKKK is phosphorylated and activated, allowing it to activate the downstream MAPKK. The MAPKK then dual phosphorylates specific threonine $(\mathrm{T})$ and tyrosine $(\mathrm{Y})$ of the $\mathrm{T}-\mathrm{X}-\mathrm{Y}$ motif present within the kinase domain of the MAPK. There are a variety of downstream cytosolic or nuclear substrates of the MAPK signaling cascade, including other protein kinases such as p90 and SGK and transcription factors such as MEF2C and Elk-1.

Source: Nithianandarajah-Jones GN, Wilm B, Goldring CE, Muller J, Cross MJ. ERK5: structure, regulation and function. Cell Signal. 2012;24(11):2187-96. 
export, sequestering inactive ERK5 to the cytoplasm (Nithianandarajah-Jones et al., 2012). Activation by MEK5 induces a conformational change in ERK5, exposing the NLS, and allowing ERK5 to translocate into the nucleus (Drew et al., 2012). Nuclear localization is crucial for ERK5's role in transcriptional activation and cell proliferation (Gomez, Erazo, \& Lizcano, 2016). While the best characterized ERK5 substrates are nuclear transcription factors such as c-FOS, c-MYC, and MEF2 family transcription factors, ERK5 does have a few cytosolic substrates including p90RSK kinases, the proapoptotic protein BAD, and the GAP junctional protein CX43 (Drew et al., 2012; Gomez et al., 2016). However, while it has been shown that inhibiting ERK5 reduces phosphorylation of these substrates, only CX43 has been confirmed to be directly phosphorylated by ERK5 (Tubita, Lombardi, Tusa, Dello Sbarba, \& Rovida, 2020).

ERK5 is ubiquitously expressed in mammalian tissues and is activated by extracellular signals such as growth factors, several cellular stressors, and oxidative phosphorylation (Khan et al., 2018; Nishimoto \& Nishida, 2006; Nithianandarajah-Jones et al., 2012). Mouse genetic studies have demonstrated that MEKK2/3-MEK5-ERK5 signaling is required for early embryogenesis, development of the vasculature and muscles, endothelial cell function, and cardio protection (Hayashi et al., 2004; W. Liu et al., 2017; Nishimoto \& Nishida, 2006; Nithianandarajah-Jones et al., 2012). Alterations in the MEKK2/3-MEK5-ERK5 pathway have been associated with several human diseases and disorders including cancer, childhood obesity, scoliosis, and cerebral cavernous malformation (Cullere, Plovie, Bennett, MacRae, \& Mayadas, 2015; Simoes, Rodrigues, \& Borralho, 2016; T. Zhou et al., 2018; Zhu et al., 2014)

\section{Summary and Aims}

Cells need to maintain sufficient quantities of healthy mitochondria to meet their metabolic demands, but it can be dangerous for cells to carry too many of these organelles. Mitophagy is essential to maintaining a population of healthy mitochondria and is needed for proper development and is necessary to preserve tissue and organ function in response to a variety of stressors (Pickles et al., 2018). Research in mitophagy has become very popular in recent years, and it is becoming clear that mitophagy is a complex cellular response that depends on several factors such as tissue type, cellular metabolic status, type of stressor, and signaling contexts. However, there is still a lot to learn about its physiological regulation and how mitophagy contributes to diseases.

In this study, I utilized a variety of methods to investigate the role of the MEKK3MEK5-ERK5 signaling cascade in basal mitochondrial degradation in mammalian cells and in erythrocyte maturation. I also explored using novel natural products to induce mitophagy. These results will potentially shed light on important biological mechanisms, therapy development, and drug design for targeting modulation of the autophagy pathway. 


\section{CHAPTER 2. MATERIALS AND METHODS}

\section{Cell Culture}

Transformed MEFs, U2OS cells stably expressing mitochondrial targeted mCherry, and GFP-LC3B, and HeLa YFP-Parkin cells were received from Michael White's laboratory. The U2OS GFP-LC3B cells were originally a kind gift from Xiaodong Wang, and the HeLa YFP-Parkin cells were originally a kind gift from Richard Youle. Cells were grown in DMEM supplemented with 10\% FBS, L-Glutamine, Penicillin and Streptomycin, and Sodium Pyruvate, and incubated at $37^{\circ} \mathrm{C}$ and $5 \% \mathrm{CO}$. Mito-mCherry, GFP-LC3B, and mito-mKeima expressing U2OS cells were maintained in $1 \mathrm{mg} / \mathrm{ml} \mathrm{G} 418$ DMEM media.

\section{Transfection}

\section{Overexpression}

Plasmid transfections were performed using Effectene (QIAGEN) according to the manufacturer's protocol. Mito-mKeima stably expressing parental and ERK5 KO

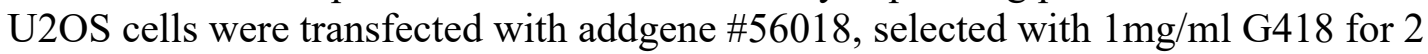
weeks, and flow sorted for high expressers.

\section{Knockdown}

Knockdown experiments for figure 3-3 were performed as described in Potts et al. 2013, but briefly HCT116 cells were transfected using Dharmafect 4 and Thermo Fisher Scientific's human siARRAY® library SMARTpools and miRNA mimics was based on the Wet Reverse Transfection Version 2.0 protocol.

All other knockdown experiments were performed using Lipofectamine RNAIMAX (Invitrogen) according to the manufacturer's protocol and with the following siRNA constructs obtained from Sigma: MISSION siRNA Universal Negative Control \#1 (SIC001), SQSTM1/P62 (SASI_Hs01_00118616, SASI_Hs01_00118618, SASI Hs01_00118620, SASI $\bar{H}$ s01_00118621), MAP3K3/MEKK3 (SASI Hs01̄ 00047592, SASI Hs0̄̄ 00047591, SASI Hs01 00047590, SASI Hs01 00047593), MAP2K5/MEK5 (SASI Hs01 00115610, SASI_Hs01_00115609, SASI_Hs01_00115611, SASI_Hs02_00334374), MAPK7/ERK5 (SASI_Hs01_00226859,SASI_Hs01_00226860,SASI_Hs01_00226861, SASI_Hs01_00226862). siRNA pools indicate that all of the oligos from the specified gene were mixed together in equal quantities before being added to cells. 


\section{CRISPR/Cas-9 Targeting ERK5}

All CRISPR/Cas-9 knockouts were kindly provided by collaborators Shondra Miller and Sadie Sakurada. SQSTM1/p62 and MAPK7/ERK5 knockout U2OS cells were generated using CRISPR-Cas9 technology. Briefly, 400,000 U2OS cells were transiently transfected with precomplexed ribonuclear proteins (RNPs) consisting of 100 pmol of chemically modified sgRNA (sgSQSTM1 5' - gagggaaagggcuugcaccg -3' or sgMAPK7 5' - agacggcgaggacggcucug - 3', Synthego) 35 pmol of Cas9 protein (St. Jude Protein Production Core), and 200ng of pMaxGFP (Lonza) via nucleofection (Lonza, 4DNucleofector $^{\mathrm{TM}} \mathrm{X}$-unit) using solution P3 and program CM-104 in a small (20ul) cuvette according to the manufacturer's recommended protocol.

Five days post nucleofection, cells were single-cell sorted by FACS to enrich for GFP+ (transfected) cells, clonally selected, and verified for the desired targeted modification via targeted deep sequencing using gene specific primers with partial Illumina adapter overhangs (hSQSTM1.F - 5' - acagccccacagtgacgacagaggg - 3' and hSQSTM1.R 5' - tgggggaggaattagcagagcggca -3' or hMAPK7.F - 5' agctagtctgccacgaaccagccgc 3' and hERK5.R - 5' cgggcggaggacaccactccatagg 3', overhangs not shown). NGS analysis of clones was performed using CRIS.py (Connelly \& Pruett-Miller, 2019). Three MAPK7/ERK5 knockout clones were identified, and genotypes are given below. SQSTM1/p62 knockout clone genotypes are available upon request.

WT 5'- aggaagacggcgaggacggctctgcggagcccccgggeccgtgaagg -3’

ERK5 KO Clones

1 -28bp 5'- aggaagacggc-----------------------ccgtgaagg -3'

+1bp 5'- aggaagacggcgaggacggetTctgcggagcccccgggccegtgaagg -3 '

$2+1 \mathrm{bp} 5$ '- aggaagacggcgaggacggctTctgcggagcccccgggeccgtgaagg -3 '

-4bp 5'- AGGAAGACGGCGAGGA----

TCTGCGGAGCCCCCCGGGCCCGTGAAGG -3’

$3+1$ bp 5'- aggaagacggcgaggacggctTctgcggagcccccgggccegtgaagg -3 '

+2bp 5'- aggaagacggcgaggacggctctCTgcggagcccccegggcccgtgaagg -3’

\section{Drug Treatments}

Unless otherwise stated cells were treated with 10uM BIX02189 (Tocris 4842) and 10uM XMD8-92 (Tocris 4132) for 16 hours and 50nM Bafilomycin A1 (Sigma B1793) for 2 hours. 


\section{Mito-mCherry Mitochondrial Content}

U2OS cells stably expressing mito-mCherry were plated in 6-well plates for flow cytometry analysis or in Nunc Lab-Tek II chambered coverglass for microscopy imaging. The cells were stained with 100nM Mito-Tracker Green FM for 30 minutes then washed with PBS three times. After the final PBS wash, cells are treated with either a DMSO control, BIX02189, or XMD8-92. Cell were then incubated for 16-24 hours and trypsinized and resuspended in Flow buffer for Flow Cytometry analysis on a BD Biosciences Fortessa or continually imaged on Multimodal 3i Marianas, then analyzed for relative fluorescence of Mito-Tracker Green FM compared to mito-mCherry.

\section{Mitochondrial Uncoupling}

HeLa YFP-Parkin cells were treated with $10 \mathrm{nM}$ CCCP overnight in addition to indicated treatments. Cells were then fixed and stained with DAPI and TOMM20 antibody.

U20S parental and ERK5 KO U2OS cells expressing mito-mKeima were treated with $10 \mathrm{nM} \mathrm{CCCP}$ overnight in addition to indicated treatments. Cells were then analyzed via flow cytometry.

\section{Confocal Imaging}

Images were taken with Leica SP8 TCS equipped with White Light Laser, $405 \mathrm{~nm}$ diode laser using 63x/1.4NA/Oil/HC PL APO CS2/0.14mm objective confocal microscope. Mito-mCherry images were created from an over-sampled Z-stack with XY pixel size of $16 \mathrm{~nm}$ and Z-step size of $100 \mathrm{~nm}$. For higher resolution images deconvolution was performed in Huygens Professional 16.10 (Scientific Volume Imaging, Netherlands). Isosurface rendered image was created in Imaris 9.3 (Oxford Instruments, UK).

GFP-LC3 puncta and SQSTM1/p62 puncta were manually counted on a per cell basis.

ERK5+/+ and ERK5 -/- U2OS cells stably expressing mitochondrial targeted mKeima were plated in Nunc Lab-Tek II chambered coverglass and imaged live on the Leica SP8 TCS confocal microscope. Data were analyzed by comparing 561 (acidic) signal to 458 (neutral) signal (Figure 2-1).

\section{Lysosomal Content and Acidification}

Lysosomal content was determined by staining with LysoTracker Red DND-99 (L7528) at 100nM for 30 minutes. Lysosomal acidification was determined by staining 


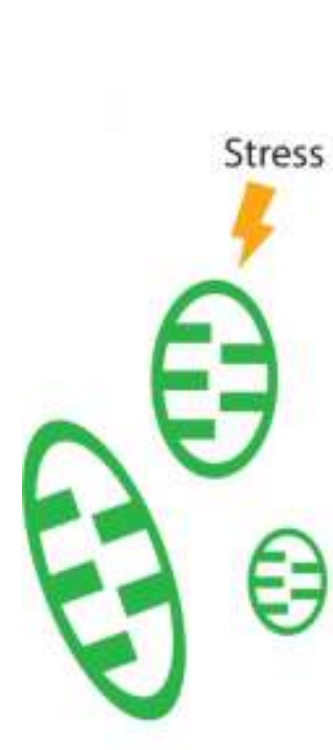

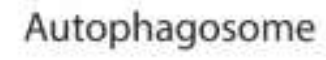
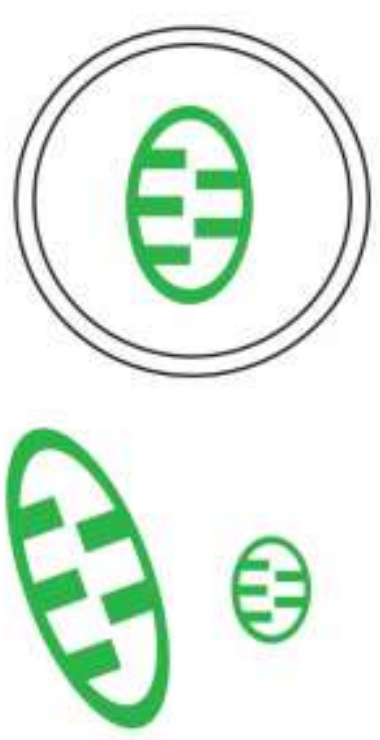

Autolysosome
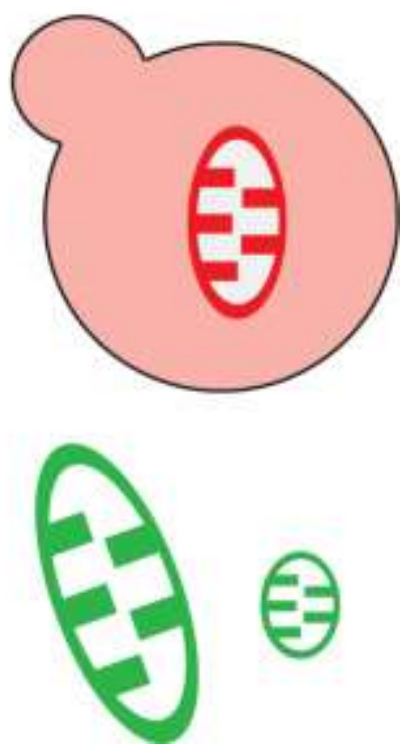

Figure 2-1. Mitochondrial targeted mKeima detects mitophagy events.

Keima is a fluorescent protein that has dual-excitation and is $\mathrm{pH}$ sensitive. Keima is also resistant to degradation by lysosomal proteases, making it ideal for use in autophagy research. Within the mitochondria $(\mathrm{pH} 8.0$ is predominately excited by $440 \mathrm{~nm}$. Within the acidic lysosome ( $\mathrm{pH}$ 4.5), Keima's excitation shifts to a longer-wavelength excitation, $586 \mathrm{~nm}$. Keima is a fluorescent protein with an excitation spectrum that changes according to $\mathrm{pH}$. Thus, when Keima is targeted to the mitochondria, the ratio of fluorescent intensity in neutral vs acidic conditions can be used to determine the level of mitophagy in living cells.

Source: Sun N, Malide D, Liu J, Rovira, II, Combs CA, Finkel T. A fluorescence-based imaging method to measure in vitro and in vivo mitophagy using mt-Keima. Nat Protoc. 2017;12(8):1576-87. 
with LysoSensor Green DND-189 (L7528) at 1mM for 10 minutes. Cells were then imaged using a BioTek Cytation 5 Cell Imaging Multi-Mode Reader. Cells were imaged using 20x objective. Signal was analyzed via relative fluorescence using Cell Profiler (McQuin et al., 2018).

\section{Flow Cytometry}

Cells were treated as indicated, trypsinized, spun down at $400 \mathrm{rpm}$, and resuspended in buffer made from 1 x PBS, $2 \%$ serum, $1 \mathrm{mM}$ EDTA, and $0.1 \%$ Sodum Azide. Cells were stained with DAPI. Fluorescence was then quantified on a BD Biosciences Fortessa by gating on single live cells and subsequently analyzed using FloJo v10.6.2.

\section{Immunoblotting}

Western blots were performed by lysing cells in 6x Laemmli loading buffer $(10 \%$ SDS, $20 \%$ glycerol, $5 \% \beta$-mercaptoethanol, 15\% 1M Tris $\mathrm{pH} 6.7,2 \mathrm{mg} / \mathrm{ml}$ bromophenol blue) separated on polyacrylamide gels, transferred to PVDF membranes and probed with antibodies against p62/SQSTM1 (CS 51145), XPB (SC-293), TOMM40 (MBL 3740), Bactin (Sigma A1978), VDAC (CS 4867S), S6K (CS 9202S), ERK5 (CS 3372S), mtCOX2 (Ab797393), MEKK3 (SC-28769), MEK5 (Invitrogen PA5-15083), PGC1 $\alpha$ (CS 2178S), pERK5 (CS 3371S).

\section{RNAseq}

RNA was extracted from cultured cells using RNeasy Kit (QIAGEN \#74104) following manufacturers' protocols. RNA concentration was measured using a NanoDrop (Thermo Fisher scientific, Waltham, MA) and the quality of RNA was determined with a bioanalyzer (Agilent Technologies, Santa Clara, CA). Libraries were prepared using the TruSeq Stranded Total RNA Library Prep Kit (Illumina, San Diego, CA) and subjected to 100 cycle paired-end sequencing on the Illumina HiSeq platform.

\section{Promega Autophagy Assay}

HEK293 autophagy reporter cells (HiBiT-HaloTag-LC3, Promega \#GA1040) were grown in in DMEM (ThermoFisher \#31053-028) supplemented with 10\% FBS (Hyclone \#SH30071), L-Glutamine (ThermoFisher \#25030-081), Penicillin and Streptomycin (ThermoFisher \#15140-122), Sodium Pyruvate (ThermoFisher \#11360070 ), and $500 \mu \mathrm{g} / \mathrm{mL} \mathrm{G} 418$ (ThermoFisher \#10131) at $37^{\circ} \mathrm{C}$ and $5 \% \mathrm{CO}_{2} .2500$ cells in $25 \mu \mathrm{l}$ of G418-free media were plated into each well of Corning 8804BC 384-well plates and grown for 18 hours. Cells were then treated with 10-point curves with 3-fold dilutions and a top concentration of $60 \mu \mathrm{M}$ of Bafilomycin A1 (positive control for 
autophagy inhibition), Rapamycin (TOCRIS \#1292; positive control for autophagy activation), BIX02188, BIX02189, and XMD8-92, or DMSO using a V\&P Scientific S100 pin-tool before they were returned to $37^{\circ} \mathrm{C}$ with $5 \% \mathrm{CO}_{2}$ for 24 hours. The assay plates were then equilibrated to room temperature for 15 minutes before the addition of $25 \mu 1$ of Nano-Glo HiBiT Lytic Detection System (Promega \#N3030.) The plates were then shaken at $300 \mathrm{rpm}$ for 2 minutes on an orbital shaker, incubated for 30 minutes at room temperature, and the luminescence of each well measured using a Perkin Elmer Envision plate reader.

\section{Erythroblast maturation}

Ter119-negative erythroid progenitors were isolated from embryonic day 13.5 or 14.5 Balb/c murine fetal livers as described (Sulahian, Cleaver, \& Huang, 2009). The purity of the cells was confirmed by staining cells with PE-CD71 and allophycocyanin (APC)-Ter119 antibodies (all from BD Biosciences) by flow cytometry. Approximately $10 \%$ of total fetal livers were purified as Ter119 negative. These progenitors were set up on retronectin coated plates for differentiation and were followed over three days for differentiation status and mitochondrial content. The data were acquired on a BD FACSCalibur and analyzed by FlowJo 8.8.4. To measure mitochondrial content, cells were stained with 25nM MitoTracker Deep Red FM (Molecular Probes, Invitrogen) for $30 \mathrm{~min}$ at $37^{\circ} \mathrm{C}$.

For inhibitor treatments starting on Day 0, Ter119-negative progenitors were set up for differentiation directly after purification in erythropoietin (Epo) media in the presence of inhibitor on retronectin coated plates. For inhibitor treatments starting on Day 2 and Day 3 Ter119-negative progenitors, 20 hours after culturing in Epo medium or 1 day after removal from EPO medium, were set up for differentiation separately, and inhibitors were added when indicated. Erythroid differentiation was monitored on days 1, 2 and 3 with PE-CD71 (BD Biosciences) and APC-Ter119 (eBioscience) by flow cytometry. 7-AAD (BD Biosciences) staining was used to exclude dead cells. Cells were treated with DMSO, $10 \mu \mathrm{M}$ BIX02188, or $10 \mu \mathrm{m}$ XMD8-92.

\section{Statistical Analysis}

Statistical analyses were performed using Graphpad Prism 8; significance was assessed by 2-tailed Student's $t$-test or by 1- or 2-factor ANOVA analysis. 


\section{CHAPTER 3. MEKK3-MEK5-ERK5 SIGNALING PROMOTES MITOCHONDRIAL DEGRADATION}

\section{SQSTM1/p62 Constitutively Delivers Mitochondria to Lysosomes for Degradation in the Absence of Exogenous Damage}

The primary question I set out to address is what controls basal mitochondrial degradation in the absence of exogenous damage. This process is known to be mechanistically distinct from damage-induced mitophagy, which requires the kinase PINK1, the E3 ligase Parkin, and the selective autophagy receptors NDP52 and Optineurin (Lazarou et al., 2015; McWilliams et al., 2018). We first investigated whether an alternative selective autophagy receptor, SQSTM1/p62, supports mitochondrial degradation in the absence of exogenous damage. We selected p62 as an initial candidate because it is not required for damage-induced, Parkin-dependent mitophagy but has been reported to be important for mitochondrial degradation in other contexts (Lazarou et al., 2015; Matsumoto, Shimogori, Hattori, \& Nukina, 2015; T. D. Nguyen et al., 2019; Yamada, Dawson, Yanagawa, Iijima, \& Sesaki, 2019; Yamada et al., 2018).

U2OS osteosarcoma cells were treated with Bafilomycin A1 for two hours to inhibit degradation of all cargo delivered to the lysosome and used immunofluorescent microscopy to determine whether such cargo included mitochondria and p62. Experiments performed by a Raju Rayavarapu revealed that a minority of the mitochondrial population (marked by TOMM20) were delivered to LAMP1-positive lysosomes in the absence of any mitophagy-inducing treatment, and all LAMP1encapsulated mitochondria were co-labeled with p62 (Figure 3-1A, B). In contrast, the vast majority of LAMP1-negative mitochondria were negative for $\mathrm{p} 62$

(Figure 3-1A, 1B). In the absence of Bafilomycin treatment we observed rare instances of p62-positive mitochondria co-localized with LAMP1 (Figure 3-1A). To determine whether lysosomal activity detectably limited mitochondrial accumulation under basal conditions, we used stably expressing mitochondrially targeted mCherry in U2OS cells (U2OS mito-mCherry cells) and measured mCherry fluorescent intensity per cell by flow cytometry as a marker of mitochondrial abundance. Short durations of lysosomal inhibition ( $<5$ hours) did not detectably increase bulk mitochondrial content per cell, while longer durations of lysosomal inhibition caused a steady increase of mitochondrial content over time (Figure 3-1C). Together these results indicate that steady-state mitochondrial abundance is limited by ongoing delivery of a small subset of p62-labeled mitochondria to lysosomes for degradation.

Treatment with Bafilomycin A1 increased total protein levels of p62 in a dose dependent manner, confirming that p62 is continually degraded by the lysosome under basal conditions (Figure 3-2A). To test whether p62 is required for basal mitochondrial degradation, I depleted p62 by RNAi and asked whether total mitochondrial content per cell increased as a result. Multiple independent siRNA oligos targeting p62 caused an increase in mitochondrial accumulation in U2OS mito-mCherry cells (Figure 3-2B, C). Similarly, Joseph Miller found that p62 knockout U2OS cells exhibited increased 
A
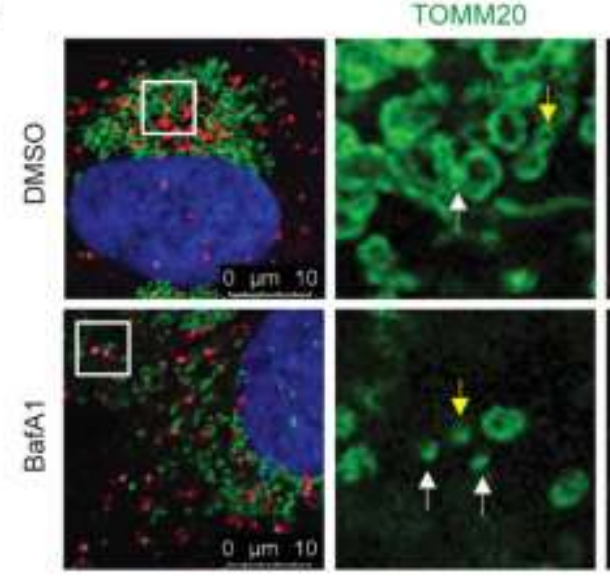

B

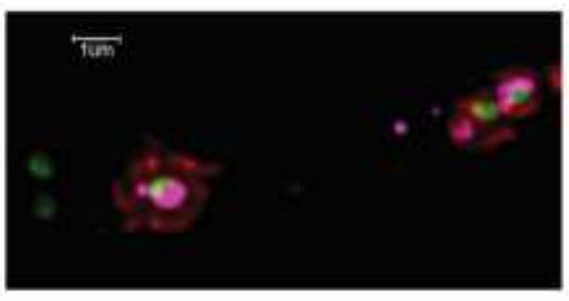

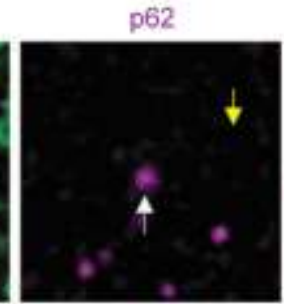
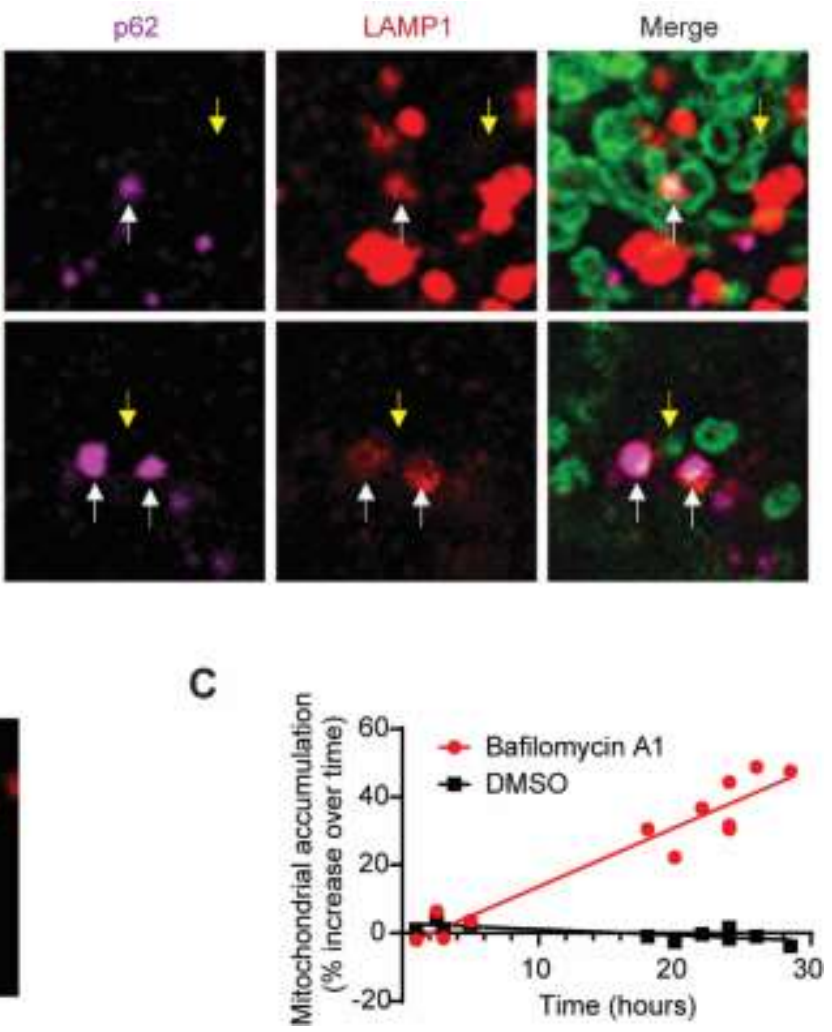

Figure 3-1. Basal mitophagy is active in U2OS cells.

(A) U2OS cells were treated with $50 \mathrm{nM}$ bafilomycin A1 for 2 hours to block degradation of lysosomal contents and analyzed by immunocytochemistry. Representative images are shown. TOMM20 (Green), p62 (purple), LAMP1 (red), DAPI (blue). Images show instances of mitochondria (TOMM20) tagged by p62 and surrounded by lysosomes (LAMP1) designated by white arrows and mitochondria that are not being degraded designated by yellow arrows. (B) Representative high-resolution STED image of cells treated as in (A). Note LAMP1-positive lysosomal membrane surrounding (top) or approaching (bottom) p62-tagged mitochondria. Tomm20 (green), p62 (red), LAMP1 (blue). (C) U2OS mito-mCherry cells were treated with $50 \mathrm{nM}$ bafilomycin A1 or vehicle (DMSO) for the indicated time. Mitochondrial accumulation was measured by flow cytometry and displayed as percent increase over time. 
A

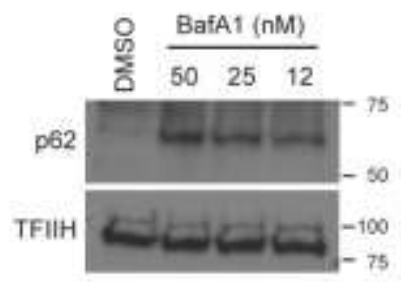

B

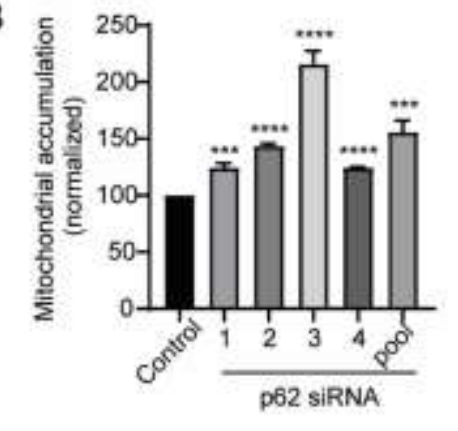

C

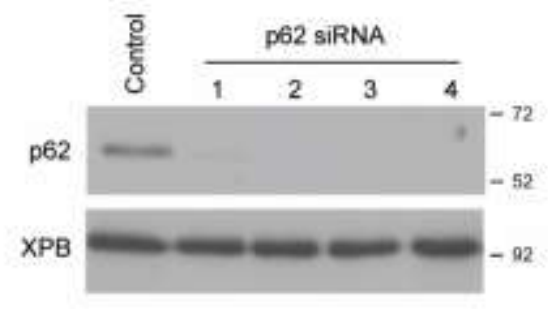

D

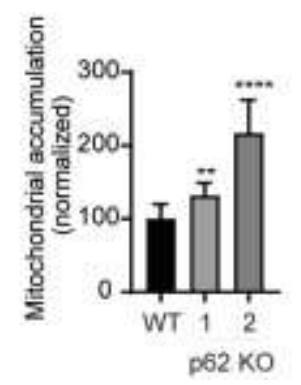

E

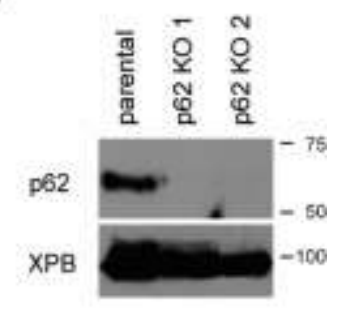

Figure 3-2. p62 is an active player in basal mitophagy.

(A) U2OS cells were treated with the indicated concentrations of bafilomycin overnight.

(B) U2OS mito-mCherry cells were transfected with the indicated siRNA oligos or siControl. (The p62 Si pool contains oligos 1-4). 72 hours later mitochondrial accumulation was measured by flow cytometry. Mean $+/-$ S.E. of $n=3$ independent experiments is shown; *** $\mathrm{p}<0.001 ; * * * * \mathrm{p}<0.0001$. (C) U2OS cells were transfected with the indicated siRNAs. 72 hours later lysates were collected and analyzed by western blot using the indicated antibodies. (D) p62 knockout U2OS cells were generated via CRISPR/Cas9-mediated genome editing. Cells were stained with $100 \mathrm{nM}$ Mitotracker Green FM for 30 minutes and mitochondrial accumulation was measured by flow cytometry. Mean +/- S.E. of $\mathrm{n}=7$ independent experiments is shown; ** $\mathrm{p}<0.01$; **** $\mathrm{p}$ $<0.0001$. (E) Parental and SQSTM1/p62 knockout U2OS cell lysates were collected and analyzed by western blot using the indicated antibodies. 
mitochondrial accumulation relative to parental U2OS cells as measured by Mitotracker Green FM staining (Figure 3-2D, E). Taken together, our data indicate p62 is important for basal delivery of mitochondria to lysosomes for degradation in the absence of exogenous damage.

\section{Identification of Novel Mitophagy Regulatory Pathway}

To identify novel mitophagy regulatory pathways, we utilized Functional Signature Ontology (FuSiOn), a method for unbiased identification of functionally related proteins, to nominate protein kinases from the human genome that function similarly to p62 under basal conditions (Potts et al., 2013). FuSiOn identified ten kinases (BMP2K, DCLK3, LIMK2, MAP2K5/MEK5, MAP3K3/MEKK3, ROS1, SIK2, TAOK2, ULK1, and ULK2) whose depletion mimicked the phenotypic effect of p62 depletion in HCT116 colon cancer cells (Potts et al., 2013). To determine whether any of these ten candidates promotes basal mitophagy, each kinase was individually depleted in U2OS mito-mCherry cells and measured the resulting change in average mitochondrial content per cell. Depletion of seven of the ten kinases caused an increase in mitochondrial content similar to that caused by depletion of p62 (Figure 3-3A). Next each kinase was depleted in U2OS GFP-LC3B cells and accumulation of GFP-LC3B as a marker of nonselective autophagy inhibition was measured. Two candidates (MAP3K3 and MAP2K5) phenocopied p62's selectivity for mitochondria, demonstrated by the relatively consistent LC3B levels in addition to the increase in mitochondrial content (Figure 3-3A). MAP3K3 encodes MEKK3, which activates the MEK5-ERK5 kinase cascade. MAP2K5 encodes the MEKK3 substrate MEK5 (Figure 3-3B).

On-target efficacy of the siRNA pools directed against MAP3K3/MEKK3 and MAP2K5/MEK5 was confirmed (Figure 3-4A). Maximum intensity projection images of U2OS mito-mCherry cells confirmed that the mito-mCherry signal remained localized to mitochondrial network after depletion of MEKK3 or MEK5 and demonstrated the accumulation of mitochondrial network in individual cells, supporting the flow cytometric data (Figure 3-4B). Next, we tested whether pharmacological inhibition of MEK5 kinase activity could alter mitochondrial abundance using two small molecule inhibitors of MEK5 (BIX02188 and BIX02189) (Tatake et al., 2008). Both BIX02188 and BIX02189 inhibited MEK5 activity and increased mitochondrial content in a dosedependent manner in varied mammalian cells (Figure 3-5A through D). Given the structural similarity between the two inhibitors, the off-target activities of BIX02188 and BIX02189 were measured by Kinome Profiling and determined that the off-target activities do not overlap (Appendix). This increases the likelihood that the observed increase in mitochondrial content is due to inhibition of the intended target, MEK5. Together, these results indicate that MEKK3-MEK5 signaling restrains mitochondrial accumulation. 
A

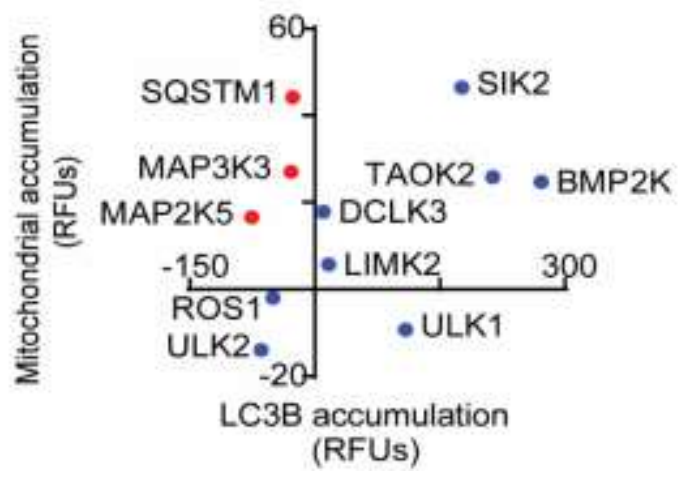

B

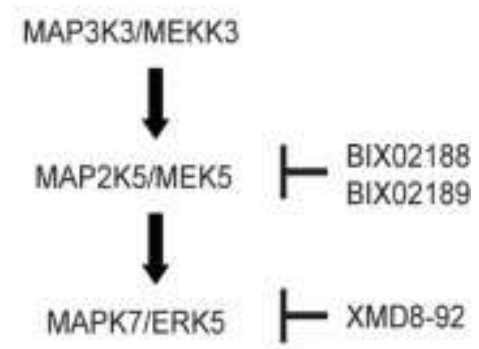

Figure 3-3 Identification of MEKK3/MEK5 as a novel pathway involved in mitophagy.

(A) Candidate mitophagy-regulating genes identified by FuSiOn were screened by siRNA-mediated depletion in U2OS mito-mCherry cells and U2OS GFP-LC3B cells. Depletion of 8 of 11 candidates caused mitochondrial accumulation (red and blue), of which only 3 exhibited selectivity for mitochondria over the bulk autophagy marker GFPLC3B (red). (B) A model of the MAP3K3 kinase cascade including inhibitors.

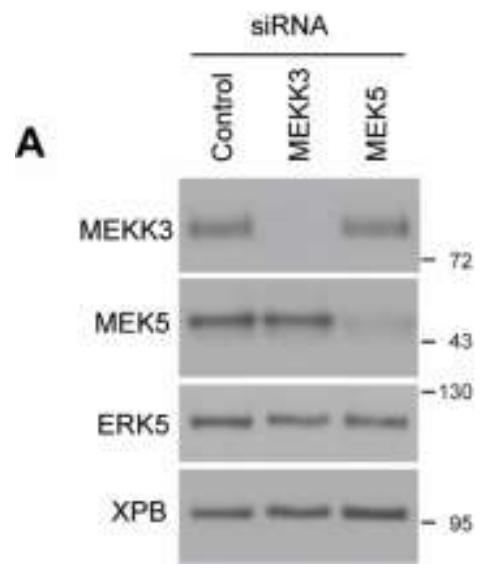

B

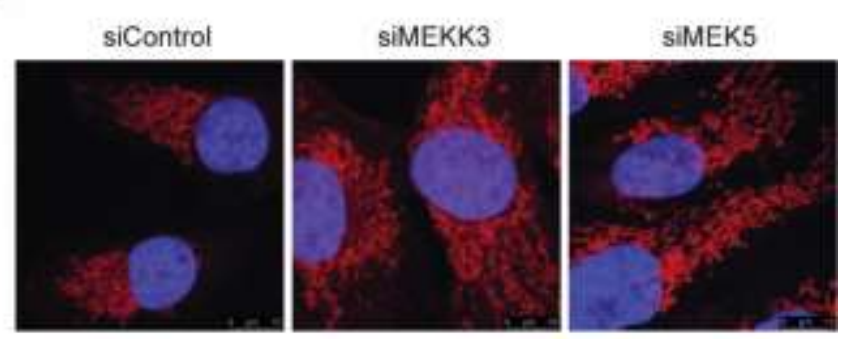

Figure 3-4. Confirmation that siRNA knockdown of MEKK3 and MEK5 increase mitochondrial content.

(A) U2OS cells were transfected with the indicated siRNAs. 72 hours later lysates were collected and analyzed by western blot using the indicated antibodies. (B) U2OS mitomCherry cells were transfected with the indicated siRNA oligos. 72 hours later cells were fixed and imaged in z-stacks. Representative maximum intensity projections are shown. 
A
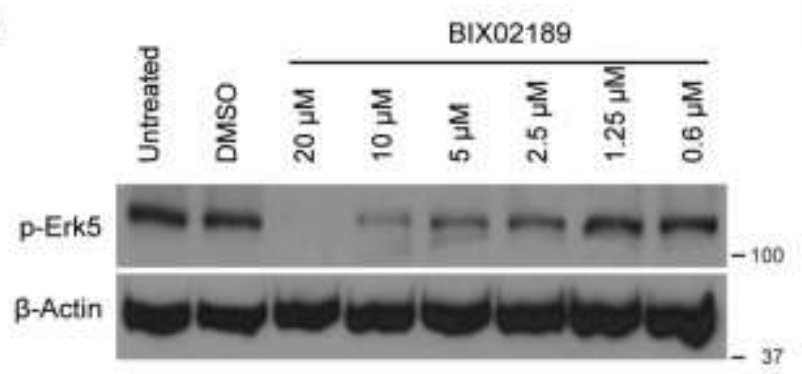

C

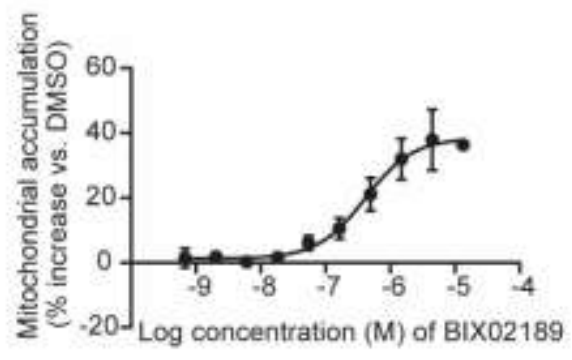

B

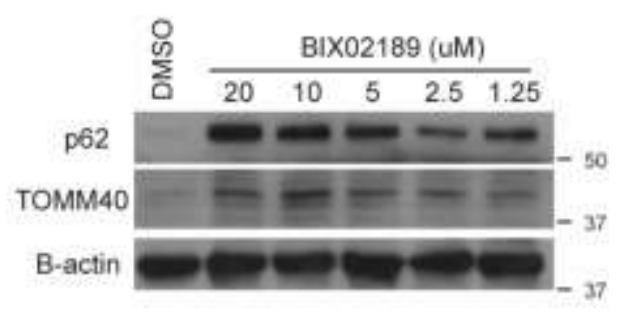

D

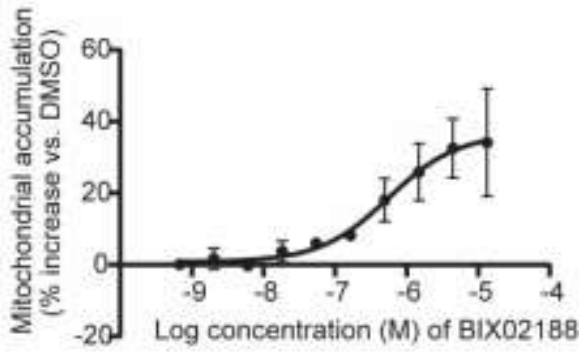

Figure 3-5. Pharmacological inhibition of MEK5 increase mitochondrial content. (A) U2OS cells were treated with vehicle (DMSO) or the indicated concentration of the MEK5 inhibitor BIX02189 overnight. Lysates were collected and analyzed by western blot using the indicated antibodies (B) U2OS mito-mCherry cells were treated with vehicle (DMSO) or the indicated concentration of BIX02189 overnight. Mitochondrial accumulation was measured by flow cytometry. Mean $+/$ S.D. of $\mathrm{n}=2$ independent experiments is shown. (C) U2OS mito-mCherry cells were treated with vehicle (DMSO) or the indicated concentration of the MEK5 inhibitor BIX02188 overnight.

Mitochondrial accumulation was measured by flow cytometry. Mean $+/$ - S.D. of $n=3$ independent experiments is shown. (D) Mouse embryonic fibroblasts were treated with vehicle (DMSO) or the indicated concentration of the MEK5 inhibitor BIX02189 overnight. 


\section{The MEKK3-MEK5-ERK5 Kinase Cascade Prevents Accumulation of Excess Mitochondria}

We proceeded to assay whether the canonical downstream component of this kinase cascade, ERK5 (encoded by the MAPK7 gene), also plays a role in restraining mitochondrial accumulation. RNAi-mediated depletion of ERK5 increased the average mitochondrial content per cell, indicating that ERK5 (like p62, MEKK3, and MEK5) prevents excess accumulation of mitochondria under basal conditions (Figure 3-6A, B). A small molecule inhibitor of ERK5, XMD8-92 (Q. Yang et al., 2010), also increased mitochondrial content in a dose-dependent manner (Figure 3-6C, D). Our collaborators, Shondra Miller and Sadie Sakura, generated MAPK7/ERK5 knockout U2OS cells using CRISPR/Cas9 technology, and I analyzed mitochondrial content via western blot and MitoTracker Green FM staining. In both cases, ERK5 knockout cells exhibited increased mitochondrial accumulation relative to the parental controls (Figure 3-6E, F). These data indicate that the canonical MEKK3-MEK5-ERK5 kinase cascade restrains mitochondrial accumulation under basal conditions.

We asked whether the MEKK3-MEK5-ERK5 kinase cascade promotes mitochondrial degradation through regulation of p62 protein levels. MEKK3, MEK5, and p62 all contain PB1 domains, which mediate protein binding with other PB1 domain containing proteins (Nakamura, Kimple, Siderovski, \& Johnson, 2010). We hypothesized that MEKK3-MEK5-ERK5 pathway inhibition might decrease p62 protein stability, or alternatively, might reduce p62 expression given that ERK5 is known to translocate to the nucleus and regulate gene transcription when activated (Drew et al., 2012; Nithianandarajah-Jones et al., 2012). In either of these cases, a decrease in p62 levels upon MEKK3-MEK5-ERK5 pathway inhibition could explain why mitochondria accumulate under those conditions.

However, genetic or pharmacological inactivation of the MEKK3-MEK5-ERK5 kinase cascade induced p62 protein accumulation rather than decrease in abundance, indicating that MEKK3-MEK5-ERK5 signaling is not required for p62 expression or stability (Figures 3-5B, 3-6D, and 3-7A). Using immunofluorescent microscopy, we found that genetic or pharmacological inactivation of the MEKK3-MEK5-ERK5 kinase cascade caused an increase in the average number of cytoplasmic p62 puncta per cell (Figure 3-7B through 7E). The punctate accumulation of the selective autophagy adaptor protein p62 upon inhibition of the MEKK3-MEK5-ERK5 pathway is consistent with the interpretation that this pathway promotes one or more forms of selective autophagy under basal conditions.

\section{The MEKK3-MEK5-ERK5 Pathway Is Required for Lysosomal Degradation of Mitochondria}

To determine the underlying cause of increased mitochondrial content observed upon inhibition of MEKK3-MEK5-ERK5 signaling, I considered and tested three distinct possibilities: 1) induction of mitochondrial biogenesis; 2) nonselective inhibition of the 
A

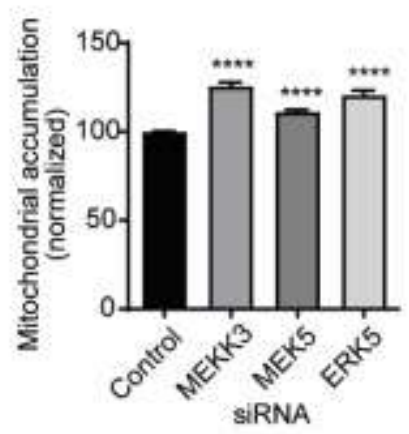

C

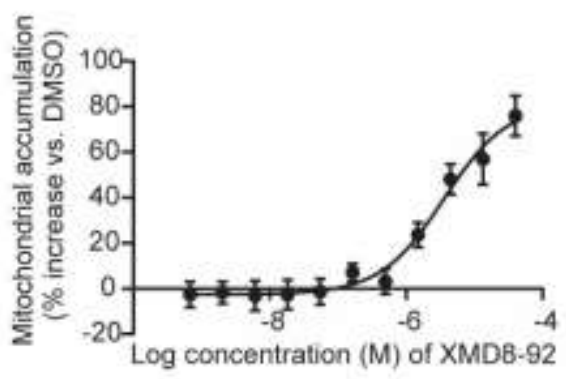

E

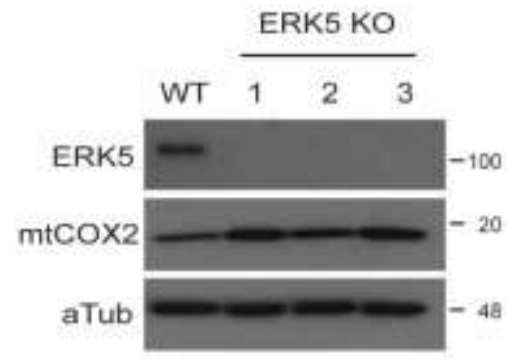

B
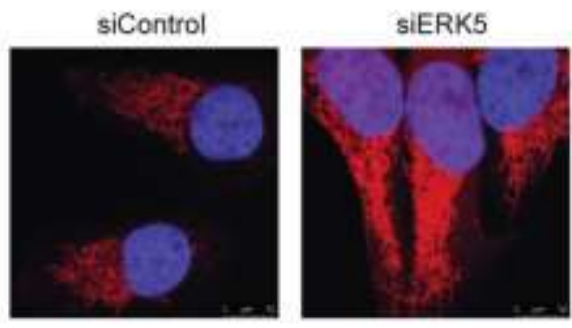

D

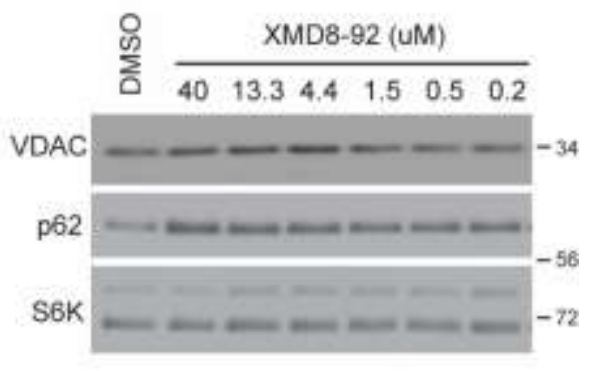

$\mathbf{F}$

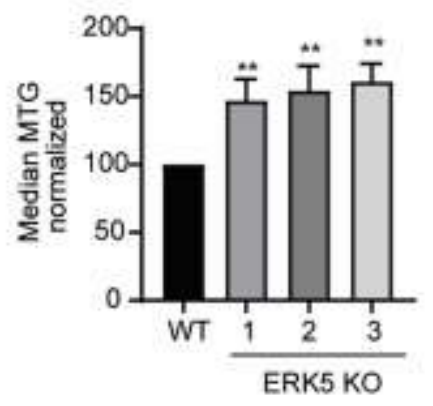

Figure 3-6. Genetic and pharmacological inhibition of downstream component ERK5 also increases mitochondrial content.

(A)U2OS mito-mCherry cells were transfected with the indicated siRNA oligos targeting. 72 hours later mitochondrial accumulation was analyzed by flow cytometry. Mean +/S.D. of $\mathrm{n}=3$ independent experiments is shown. (B) U2OS mito-mCherry cells were transfected with the indicated siRNA oligos. 72 hours later cells were fixed and imaged in z-stacks. Representative maximum intensity projections are shown. (C, D) U2OS mito-mCherry cells were treated with vehicle (DMSO) or the indicated concentration of XMD8-92 overnight. Mitochondrial accumulation was measured by flow cytometry or western blot, respectively. Mean $+/$ S.D. of $\mathrm{n}=2$ independent experiments is shown. (E, F) Three independent MAPK7/ERK5 knockout U2OS clones were generated by CRISPR/Cas9. Mitochondrial levels were assessed by western blot using the indicated antibodies or by Mitotracker Green FM staining and flow cytometry. 
A

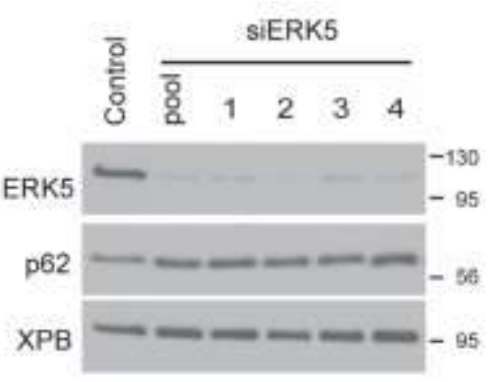

C

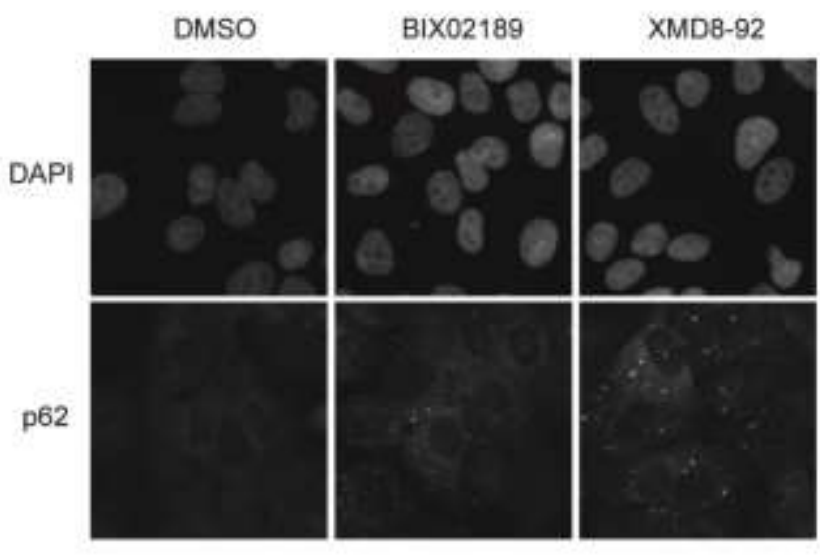

$\mathbf{E}$

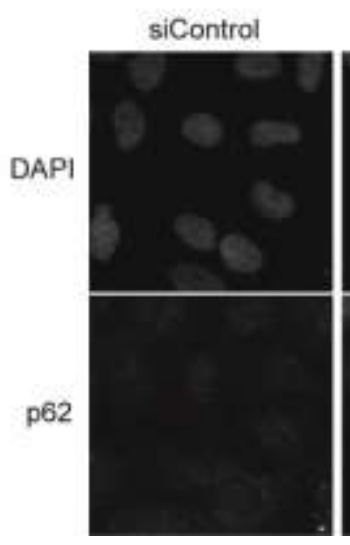

siMEKK3
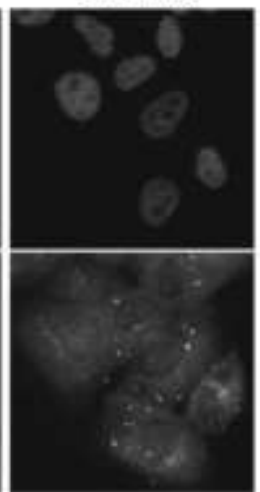

siERK5

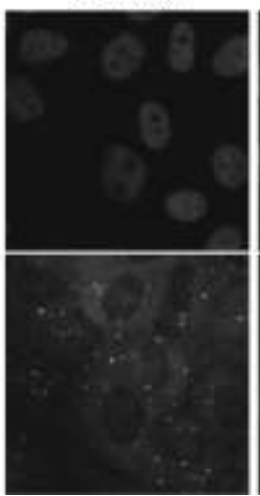

B

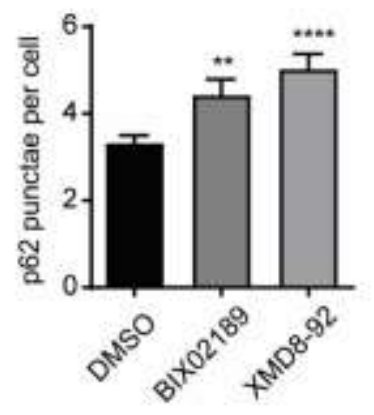

D

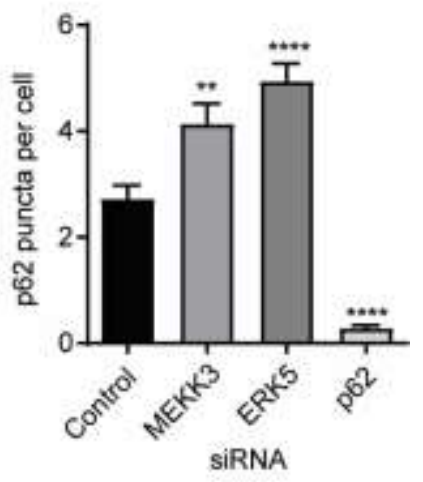

Figure 3-7. Pharmacological and genetic perturbation of the MEKK3/MEK5/ERK5 pathway cause accumulation of the SAR p62.

(A) U2OS cells were transfected with the indicated siRNAs. 72 hours later lysates were collected and analyzed by western blot using the indicated antibodies. (B, C) U2OS cells were treated with the indicated drug at $10 \mu \mathrm{M}$ overnight. Cells were fixed and stained with p62 antibody, and then imaged. (D, E) U2OS cells were transfected with the indicated siRNAs for 72 hours. Cells were fixed and stained with p62 antibody, and then imaged. 
autophagy-lysosome pathway; and 3) selective inhibition of mitochondrial degradation. PGC1 $\alpha$ is a critical regulator of mitochondrial biogenesis (Jornayvaz \& Shulman, 2010), and increased protein levels and activation of PGC1 $\alpha$ leads to an increase in the transcription of the transcription factors, TFAM, TFB1M, and TFB2M (Villarroya, Giralt, \& Villarroya, 2009). Therefore, if inhibition of MEKK3-MEK5-ERK5 signaling increases mitochondrial abundance by inducing mitochondrial biogenesis, I should detect increased expression of PGC1 $\alpha$, TFAM, TFB1M, and TFB2M upon inhibition of the pathway. RNA-seq confirmed that there was no increase in the mRNA levels of these major mitochondrial biogenesis related transcription factors upon knockout of ERK5 (Figure 3-8A). I also verified that the protein levels of the master mitochondrial biogenesis factor PGC1 $\alpha$ did not increase upon inhibition of the MEKK3-MEK5-ERK5 pathway. In fact, PGC1 $\alpha$ levels decreased upon pharmacological inhibition of MEK5 or ERK5 (Figure 3-8B), consistent with existing literature reporting a positive role for ERK5 signaling in promoting mitochondrial biogenesis (W. Liu et al., 2017). Therefore MEKK3-MEK5-ERK5 signaling does not restrain mitochondrial content by limiting mitochondrial biogenesis.

Additionally, MEK5-ERK5 signaling is not required for nonselective autophagy or for general lysosomal function. Wei Zhuang performed a Promega Autophagy Assay, in which we treated HEK293 cells expressing the HiBiT-LC3 autophagy reporter with a range of concentrations of DMSO, Bafilomycin A1, Rapamycin, BIX02188, BIX02189, and XMD8-92 for 24 hours. As expected, Bafilomycin A1 effectively inhibited nonselective autophagy and Rapamycin effectively induced autophagy across wide dose ranges, as evidenced by increased (Bafilomycin) or decreased (rapamycin) accumulation of the HiBiT-LC3 reporter (Figure 3-9A). In contrast, BIX02188, BIX02189, and XMD8-92 did not inhibit nonselective autophagy even at concentrations as high as 60 $\mu \mathrm{M}$ (Figure 3-9A). We also monitored autophagosome formation and autophagic flux using U2OS GFP-LC3B cells and observed no disruptions in either process upon treatment with XMD8-92 (Figure 3-9B, C). Furthermore, I determined that inhibition of the MEK5-ERK5 pathway does not cause lysosomal deacidification or alter lysosomal content (Figure 3-9D, E). The combined findings indicate the MEKK3-MEK5-ERK5 kinase cascade restrains mitochondrial accumulation independent of mitochondrial biogenesis, driving bulk autophagy, or supporting general lysosome function.

Next I questioned whether the MEKK3-MEK5-ERK5 kinase cascade is required for selective degradation of mitochondria. Therefore, I developed WT and ERK5 KO U2OS cell lines expressing mitochondrial-targeted monomeric Keima, a pH dependent fluorescent protein (Kogure, Kawano, Abe, \& Miyawaki, 2008; Tantama, Hung, \& Yellen, 2011) to measure the delivery of mitochondria to lysosomes (Figure 2-1). When targeted to the mitochondria, the $\mathrm{pH}$ dependent fluorescence allows us to distinguish between mitochondria in the cytoplasm $(458 \mathrm{~nm})$ and mitochondria that are being degraded in the highly acidic lysosome (561nm) (Biel \& Rao, 2018). I found that all three independent ERK5 knockout clones had significantly less mitochondria in lysosomes compared to parental cells (Figure 3-10A, B). Furthermore, in parental U2OS mitomKeima cells pharmacological inhibition of ERK5 using XMD8-92 decreased the amount of basal mitophagy compared to DMSO control (Figure 3-10C). Though 
A

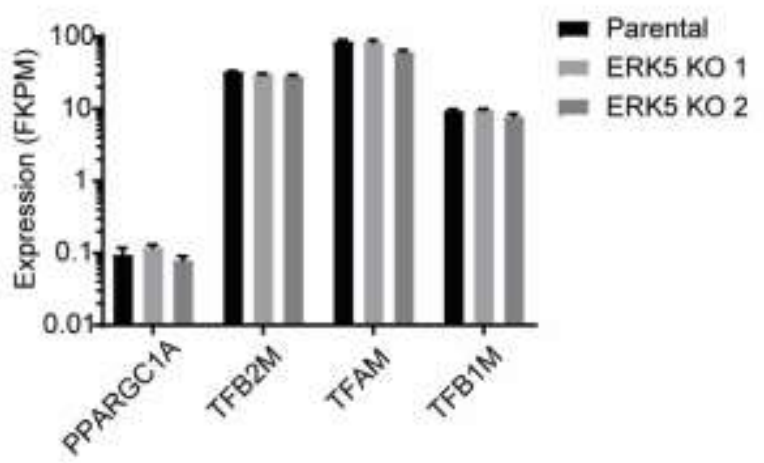

B

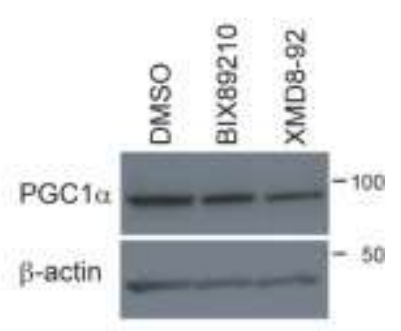

Figure 3-8. Perturbations of the MEKK3/MEK5/ERK5 pathway do not induce mitochondrial biogenesis.

(A) RNAseq was performed on parental U2OS cells and two MAPK7/ERK5 knockout U2OS clones. Expression of selected mRNAs encoding mitochondrial biogenesis genes is shown. Note that expression of these genes is not elevated in ERK5 knockout cells relative to parental controls. (B) U2OS cells were treated with vehicle (DMSO) or $10 \mu \mathrm{M}$ of the indicated compound overnight. Lysates were collected and analyzed by western blot with the indicated antibodies. 
A

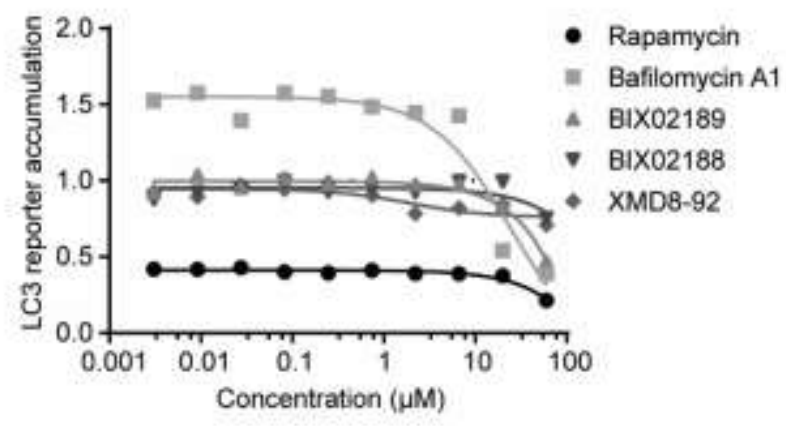

B

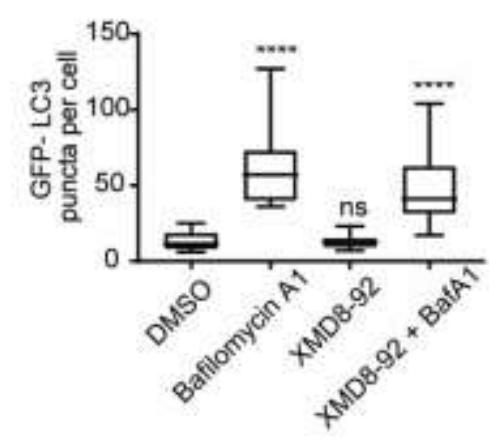

C

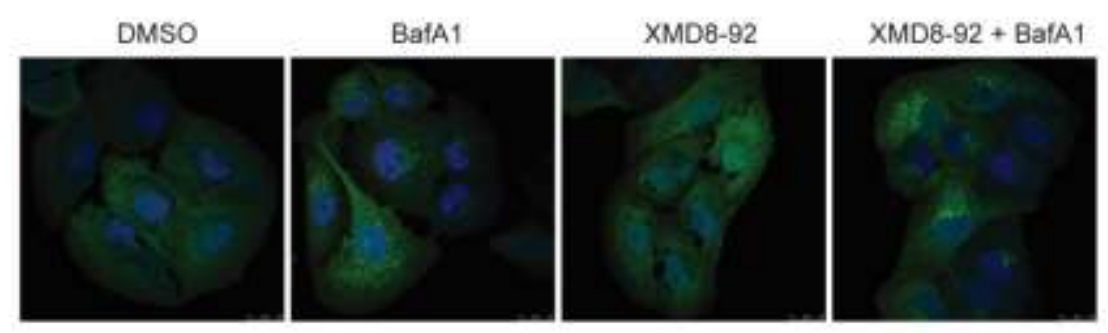

D

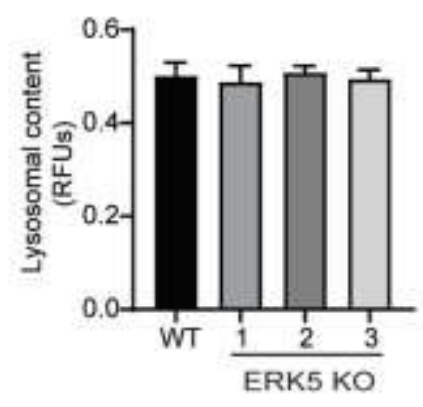

E

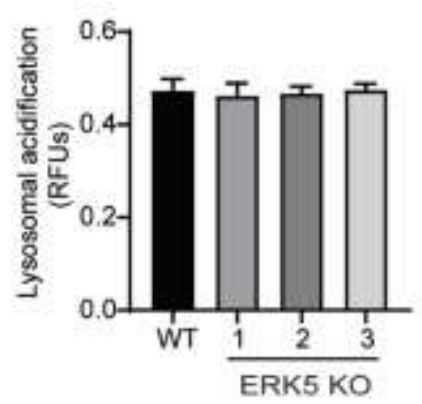

Figure 3-9. The MEKK3/MEK5/ERK5 pathway does not promote autophagy non-selectively.

(A) HEK293 cells stably expressing the HiBiT-LC3 bulk autophagy reporter were treated with vehicle (DMSO) or the indicated compounds at the indicated concentrations for 24 hours in duplicate. Accumulation of the reporter was measured using the Promega Autophagy Assay system and normalized to vehicle-treated cells. (B, C) U2OS GFPLC3B cells were treated with DMSO, XMD8-92 $(10 \mu \mathrm{M})$, Bafilomycin A1 (50 nM), or XMD8-92 + Bafilomycin A1 for two hours, fixed, stained with DAPI (blue), and imaged to assess the subcellular localization of GFP-LC3B. Representative images and quantitation of the number of GFP-LC3B-positive puncta per cell from 19-21 cells per condition are shown. (D, E) WT and ERK5 KO U2OS cells were plated in a 96 well plate. Lysosomal content and acidification were measured by staining with LysoTracker Red and LysoSensor Green respectively. Cells were then imaged, and relative fluorescence was quantified. 


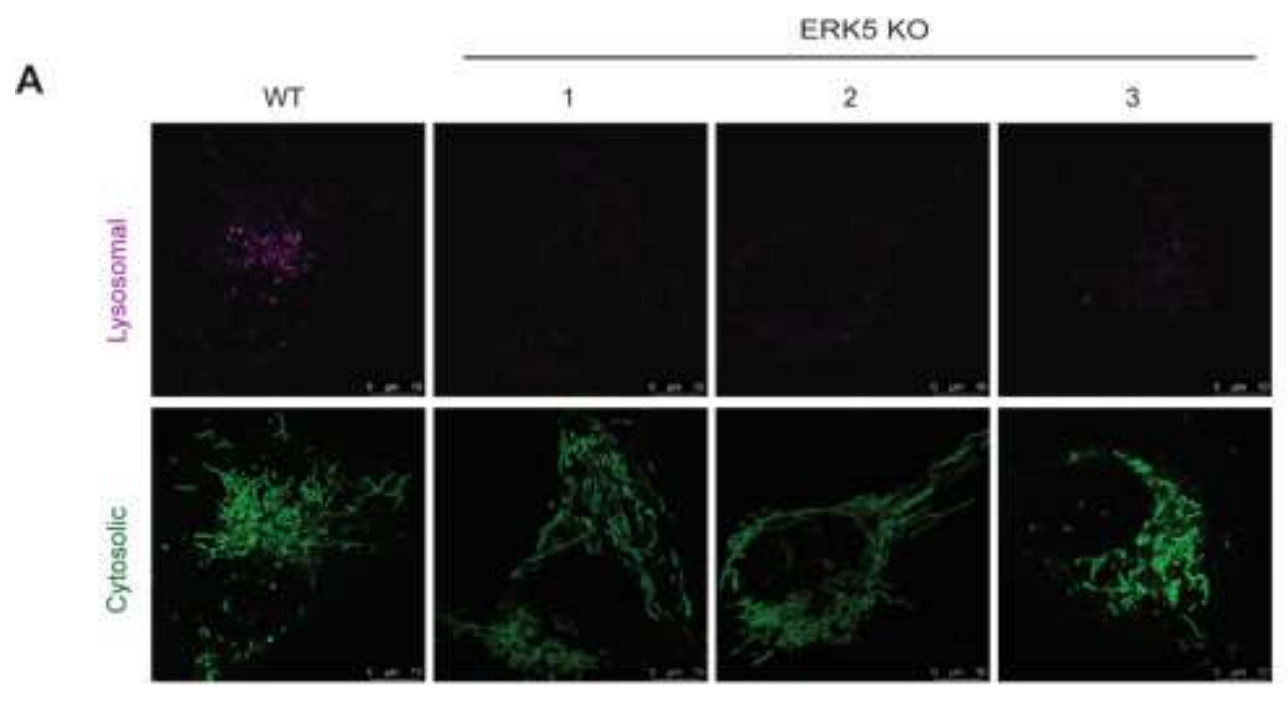

B

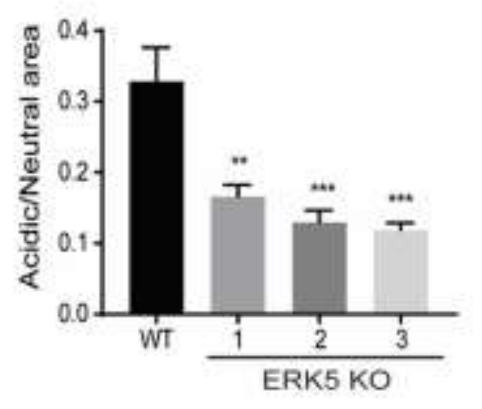

C

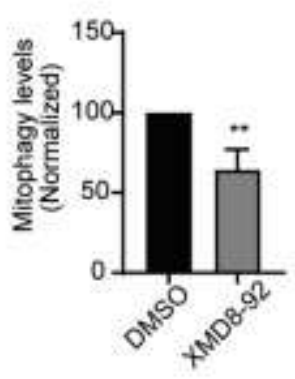

D

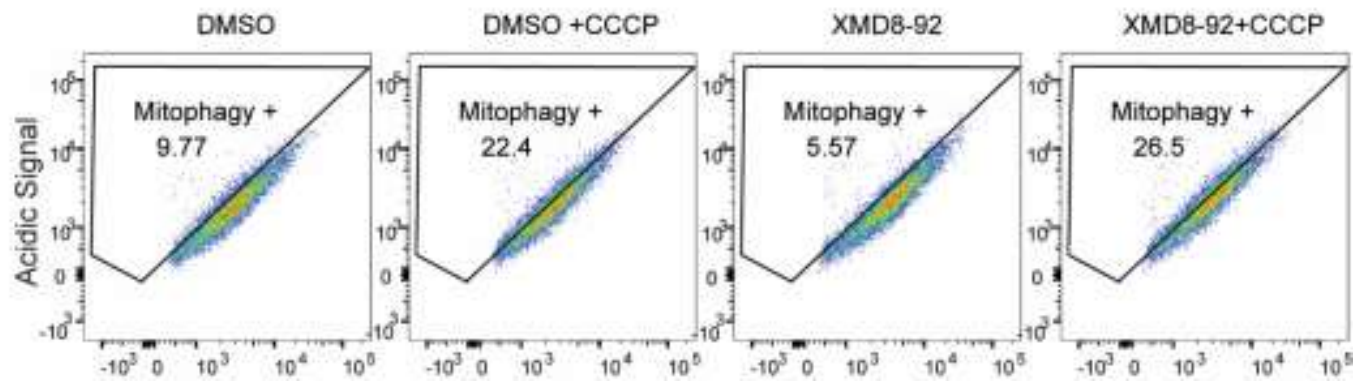

Figure 3-10. The MEKK3-MEK5-ERK5 pathway is required for lysosomal degradation of mitochondria.

(A, B) Parental (WT) and ERK5 knockout U2OS cells were transiently transfected with mitochondrial targeted mKeima. Florescence activated cell sorting was used to select transfection-positive cells which were then analyzed by fluorescent microscopy.

Representative images and quantification of active mitophagy events are shown. (C) WT U2OS cells stably transfected with mito-mKeima were treated overnight with vehicle control or $10 \mu \mathrm{M}$ XMD8-92. Cells were then collected and analyzed via flow cytometry. (D) WT U2OS cells transfected with mitochondrial targeted mKeima were treated for 13 hours with DMSO or $10 \mu \mathrm{M}$ XMD8-92. Three hours before analysis CCCP was added. Cells were assessed by flow cytometry and mitophagy levels were quantified. Mean $+/-$ S.D. of $\mathrm{n}=3$ independent experiments is shown. 
treatment with mitochondrial uncoupler, CCCP, increased mitophagy in U2OS mitomKeima cells, co-treatment with XMD was not able to mitigate the mitophagy induction (Figure 3-10D).

We were curious whether the MEKK3-MEK5-ERK5- kinase cascade had a role in PINK1-parkin mediated mitophagy in high parkin expressing cells. First, we confirmed that treating YFP-parkin expressing HeLa cells with CCCP did indeed strongly induce mitophagy (Figure 3-11A, B). When inhibiting the MEKK3-MEK5ERK5 pathway using genetic or pharmacological tools, we did not see mitigated CCCPinduced mitophagy in the presence of ectopic Parkin overexpression in HeLa cells (Figure 3-11C through $\mathbf{E}$ ). The combined data indicate the MEKK3-MEK5-ERK5 pathway specifically promotes lysosome-mediated mitochondrial degradation under basal conditions and is not required for nonspecific bulk autophagy, general lysosome function, or damage-induced mitophagy nor for restraint of mitochondrial biogenesis.

\section{MEKK3-MEK5-ERK5 Pathway is Required for Differentiation and Mitophagy in Erythroid Progenitors}

Previous studies suggest that ERK5-deficient cells develop altered nucleotide metabolism, impairing erythropoiesis in mice (Angulo-Ibanez et al., 2015).

Erythropoiesis involves an ordered acquisition and loss of key differentiation markers (Koulnis et al., 2011). During this process, erythroid progenitors must eliminate their mitochondria in order to fully mature. We collaborated with Lily Huang at UTSW to evaluate whether MEK5-ERK5 regulated mitochondrial degradation participates in erythrocyte maturation (Moras, Lefevre, \& Ostuni, 2017). To investigate the possible role of the MEKK3-MEK5-ERK5 pathway in erythrocyte maturation, Ter119-negative erythroid progenitors were isolated from murine fetal livers and induced to differentiate by addition of erythropoietin ex-vivo. We followed the expression of two specific differentiation markers: CD71, the transferrin receptor, whose expression decreases as erythroblasts mature, and Ter119, an antigen expressed on cell surfaces of more mature erythroblasts (Koulnis et al., 2011). BIX02188 or vehicle control (DMSO) were present throughout the ex-vivo differentiation process. Pharmacological inhibition of MEK5 impaired erythroid differentiation as evidenced by reduced percentages of cells reaching the most advanced Ter $119^{+} \mathrm{CD} 71^{\text {low }}$ stage of differentiation (Figure 3-12A). Notably, MEK5 inhibition significantly increased basal mitochondrial content at all stages of differentiation (Figure 3-12B). As differentiation progressed, both BIX02188-treated and DMSO-treated cells lost mitochondrial content over time; however, mitochondrial content was always significantly higher in BIX02188-treated cells relative to DMSOtreated cells at each stage (Figure 3-12B). Similar results were observed when ERK5 was inhibited using XMD8-92 (Figure 3-13A, B). Erythroid differentiation was also inhibited by genetic depletion of MEK5 using shRNA (Figure 3-14). Together, these data indicate that MEK5-ERK5 signaling is required for proper erythroid differentiation and promotes clearance of mitochondria. 
A

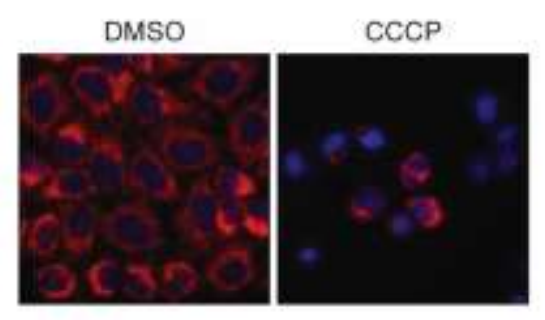

C

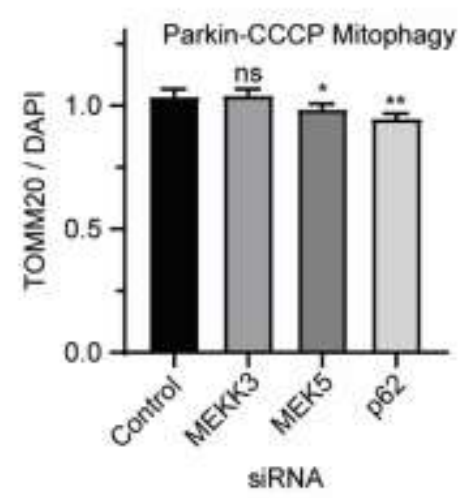

$E$

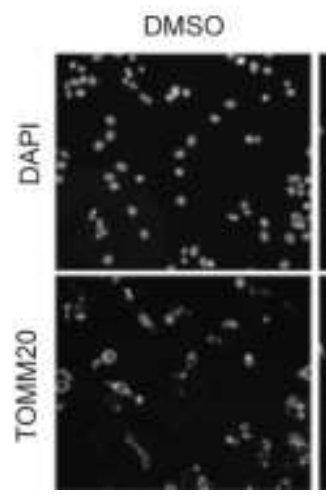

B

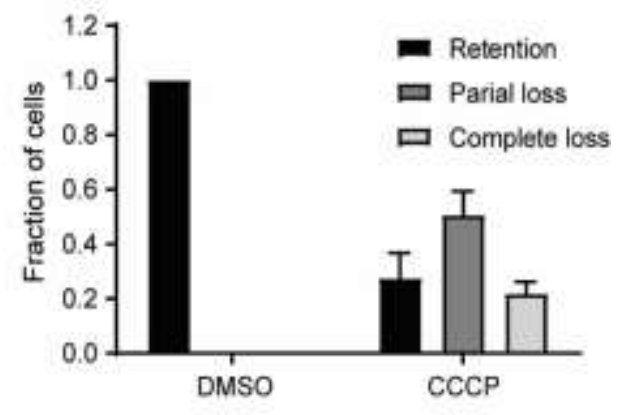

D

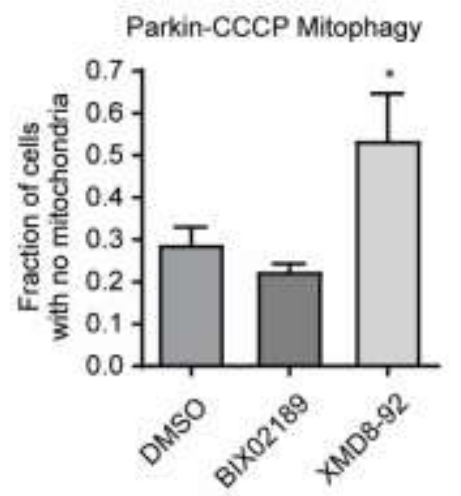

Figure 3-11. The MEKK3-MEK5-ERK5 pathway is not required for CCCPinduced Parkin-mediated mitophagy.

(A) HeLa YFP-parkin stable cells were transfected with the indicated siRNA for 72 hours and treated with $10 \mu \mathrm{M}$ CCCP overnight. Cells were fixed, stained with anti-Tom20 and DAPI, and imaged. Mitochondrial content was quantified and normalized to DAPI.

(B, C) HeLa YFP-Parkin stable cells were treated with either vehicle control (DMSO) or $10 \mu \mathrm{M}$ CCCP overnight. Cells were fixed, stained with anti-TOMM20 (red) and DAPI (blue), and imaged. Individual cells were categorized using TOMM20 fluorescence signal as exhibiting either mitochondrial retention, partial loss, or complete loss. Quantitation and representative images are shown. (D, E) HeLa YFP-Parkin stable cells were cotreated with $10 \mu \mathrm{M} \mathrm{CCCP}$ and vehicle control, BIX02189, or XMD8-92 overnight. Cells were fixed, stained with anti-TOMM20 and DAPI, and imaged. The fraction of individual cells exhibiting complete mitochondrial loss under each condition is quantified and representative images are shown. 
A
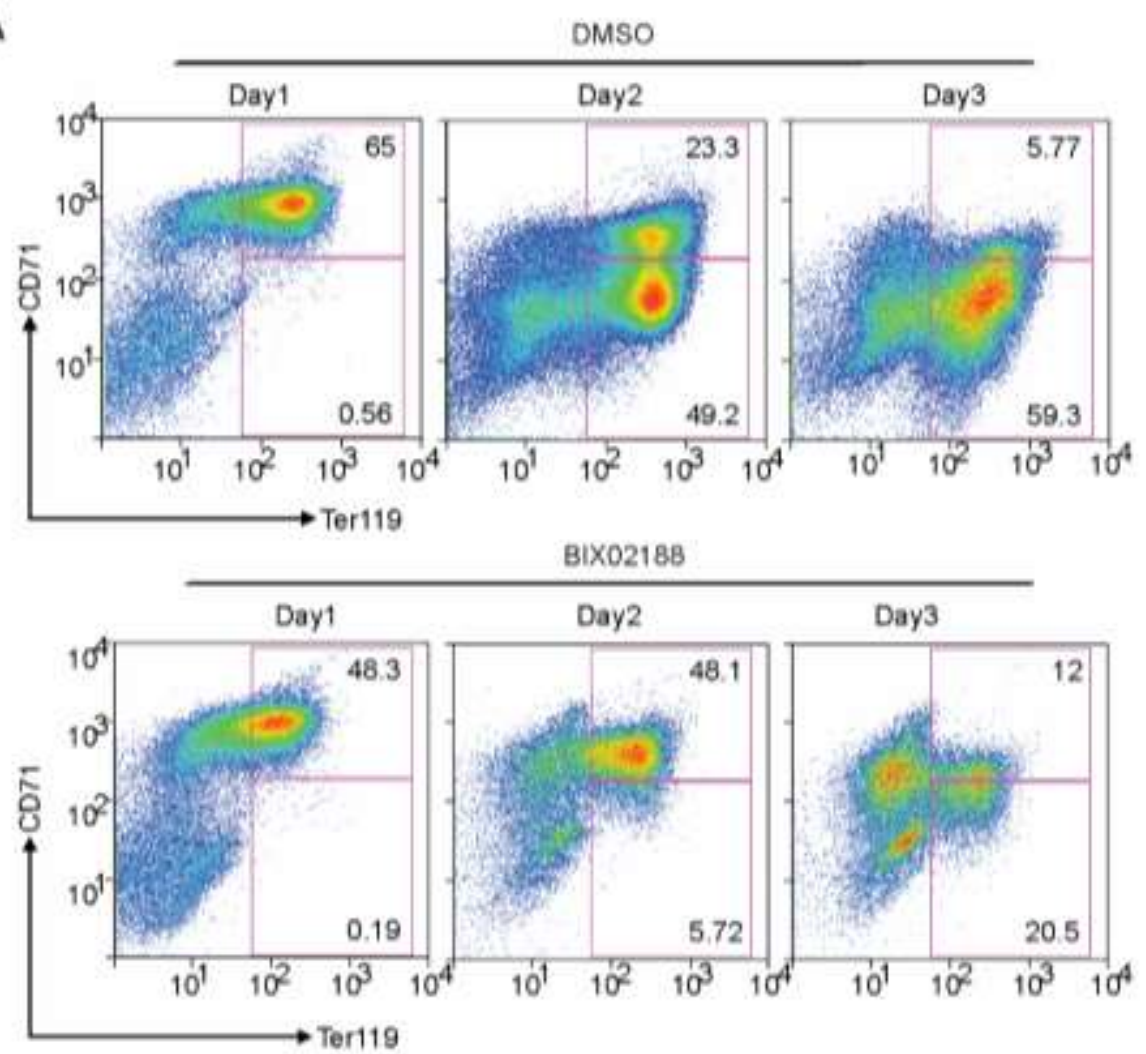

B
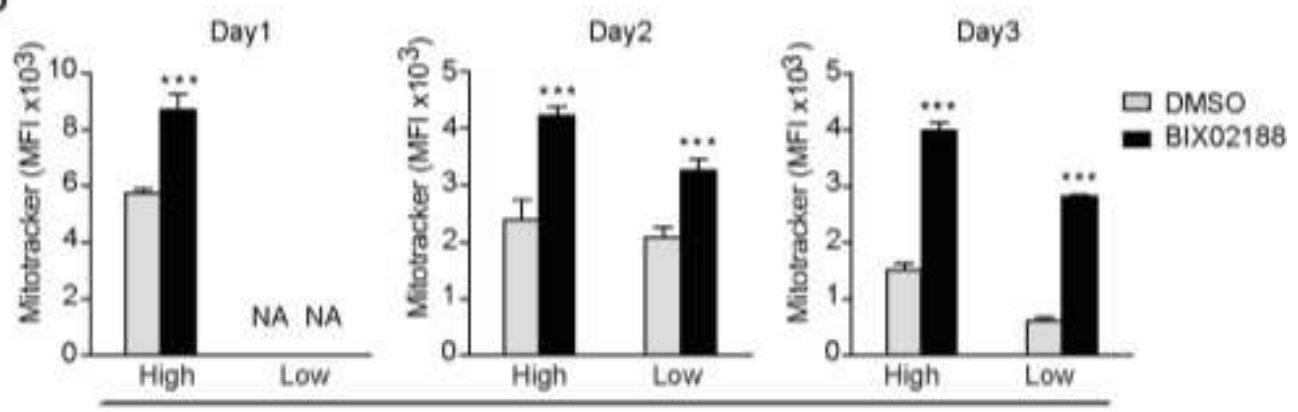

CD71 levels

Figure 3-12. MEK5 is required for differentiation and mitophagy in erythroid progenitors.

(A) Pharmacological inhibition of MEK5 impairs erythroid differentiation. Murine erythroid progenitors were treated with the Mek5 inhibitor BIX02188 (30 $\mu \mathrm{M})$ or vehicle control (DMSO) prior to initiation of differentiation. Representative profiles are shown. (B) Inhibition of MEK5 impairs mitochondrial clearance during erythroid differentiation. Mitochondrial mass was quantified in Ter119+CD71high and Ter119+CD71low erythroblasts using median fluorescence intensity (MFI) of MitoTracker Deep Red FM. The data represent three replicates. $* * * \mathrm{P}<0.001$. 
A
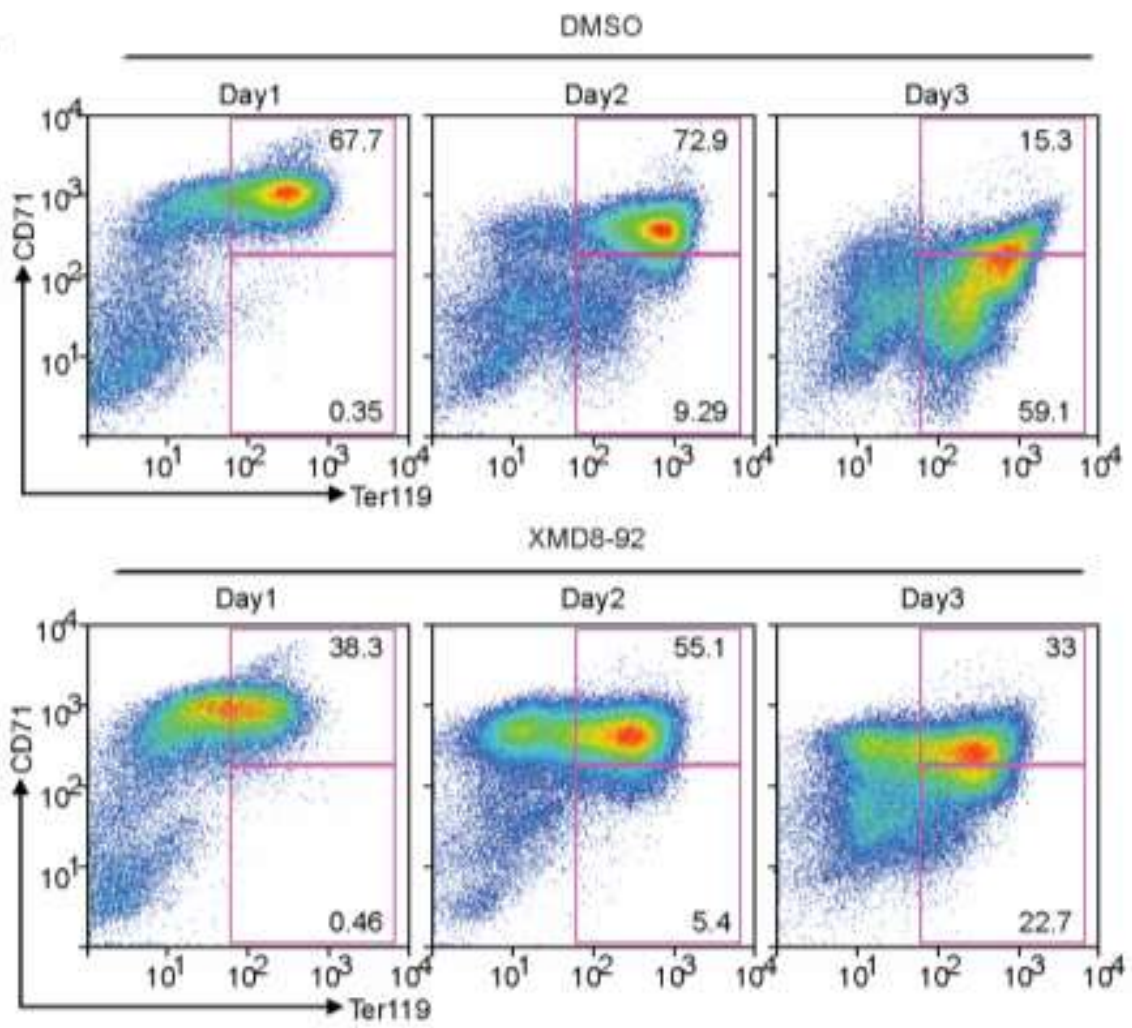

B

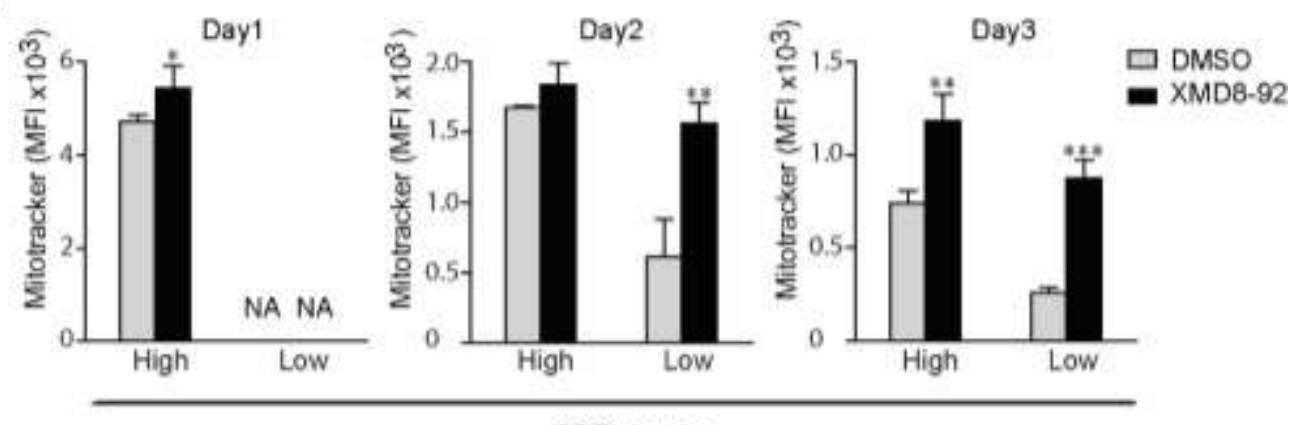

CD71 levels

Figure 3-13. ERK5 is required for differentiation and mitophagy in erythroid progenitors.

(A) Murine erythroid progenitors were treated with the ERK5 inhibitor XMD8-92 $(5 \mu \mathrm{M})$ or vehicle control (DMSO) prior to initiation of differentiation. Representative profiles are shown. (B) Inhibition of ERK5 impairs mitochondrial clearance during erythroid differentiation. Mitochondrial mass was quantified in Ter119+CD71high and Ter119+CD71low erythroblasts using median fluorescence intensity (MFI) of MitoTracker Deep Red FM. The data represent three replicates. ${ }^{*} \mathrm{P}<0.05 ;{ }^{* *} \mathrm{P}<0.01$; $* * * \mathrm{P}<0.001$ 


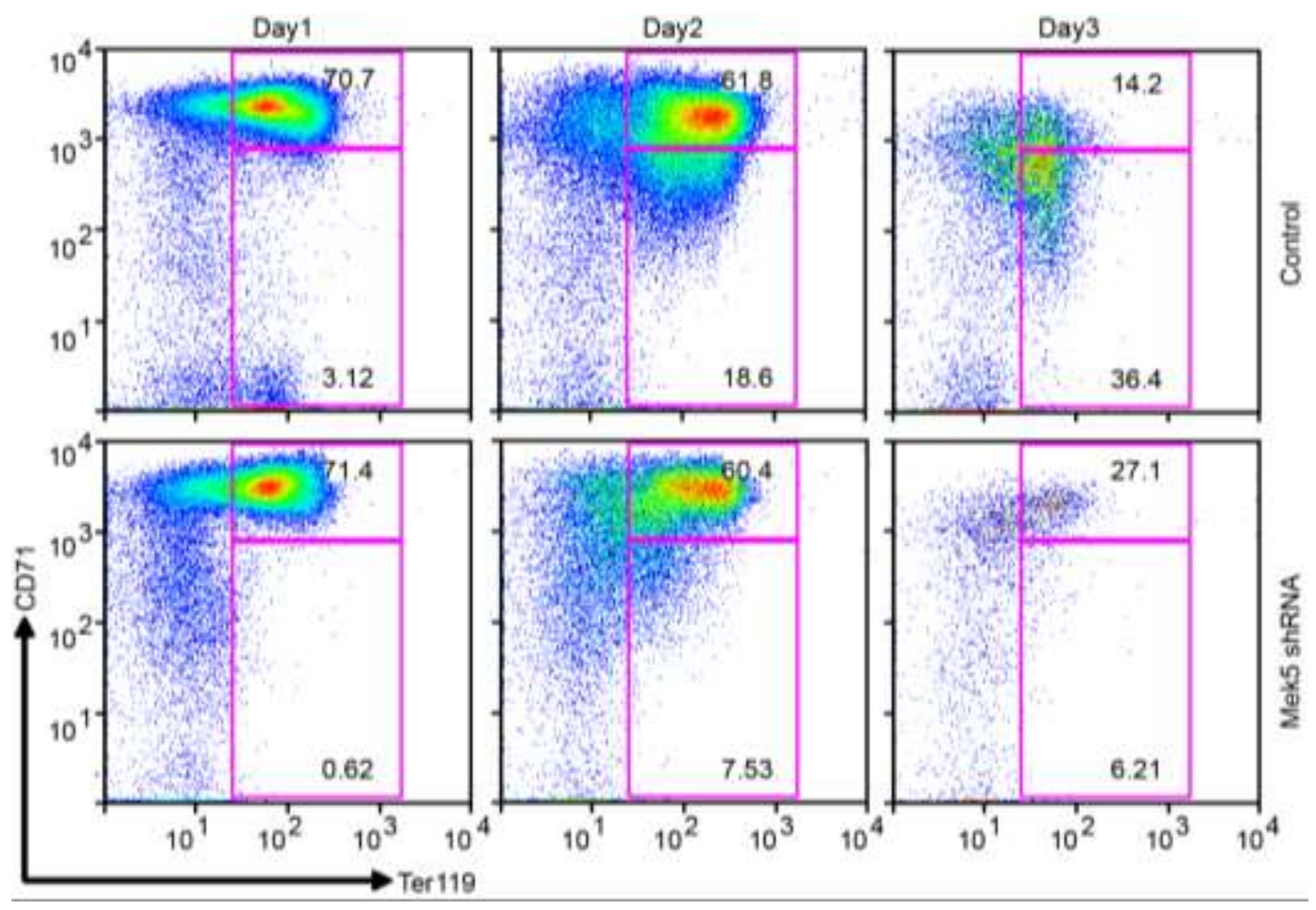

Figure 3-14. Knockdown of MEK5 impairs erythroid differentiation.

MEK5 knockdown impairs differentiation of murine erythroid progenitors. Erythroid progenitors were transduced with vectors expressing shRNA for MEK5 or control shRNA prior to erythroid differentiation. Stages of differentiation were identified by CD71 and Ter119 staining via flow cytometry. Transduced cells, identified by GFP expression, were gated for analyses. Representative flow cytometric profiles are shown. 


\section{CHAPTER 4. IDENTIFYING JATROGOSSONE A AS A NOVEL MITOPHAGY INDUCER}

There is a long history of humans using naturally occurring compounds within microorganisms, plants, or animals for their pharmacological properties. Modern research can expand on these properties by using natural products as a form of molecular inspiration for further drug and molecular probe development (Harvey, Edrada-Ebel, \& Quinn, 2015). My focus on natural products is to explore them as a source of new chemical matter to study mitophagy. To accomplish this, I collaborated with the lab of Fatima Rivas, which focuses on identifying novel natural products as sources of anticancer agents, particularly for the treatment of drug resistant cancers.

The Rivas lab conducted a high throughput screen of 100 terrestrial plant extracts including several from the Euphorbia genus. The genus contains more than 2000 species (Shi, Su, \& Kiyota, 2008) . Plants from the genus Euphorbia have been used as medicinal plants to treat illnesses such as skin diseases, gonorrhea, migraines, and intestinal parasites (Shi et al., 2008). Additionally, several classes of natural products have been found to be produced by plants in the Euphorbia genus. These natural products have a wide variety of biological properties including antiproliferative activity, inhibition of allergic reaction, antimalarial activity, and antimicrobial activity (Hadi et al., 2013; Shi et al., 2008).

For this study, the Rivas lab extracted secondary metabolites from the plants and then fractionated and evaluated them for their biological properties against drug sensitive and resistant leukemia cells as previously described (Hadi et al., 2013). Several fractions from the extract of Jatropha gossypifolia L. were identified as active hits. Jatrophone was the most abundant isolated compound (Ling et al., 2019). The Rivas lab and others have shown the promise of this compound in mechanistic studies; however, its narrow therapeutic index limits its potential therapeutic value (Bueno et al., 2015; Hadi et al., 2013). They then identified some related compounds which also displayed biological properties and focused on the natural product termed Jatrogossone A (JAX), which at the time had not been reported (Ling et al., 2019).

We went on to show that treatment with Jatrogossone A resulted in increased total ROS co-localizing in the mitochondria (Ling et al., 2019). Uncontrolled ROS can lead to damaged mitochondria and loss of membrane potential which will induce mitophagy to eliminate the effected mitochondria. First, I compared mitochondrial physiology of Jatrophone treated cells with those of Jatrogossone A treated cells (Figure 4-1). U2OS cells were treated with DMSO, $1.25 \mu \mathrm{M}$ Jatrophone (JA), or $1.25 \mu \mathrm{M}$. Jatrogossone A (JAX). Mitochondria in the JA treated sample display very aberrant morphology while in the JAX treated sample mitochondria appear to be much healthier. 

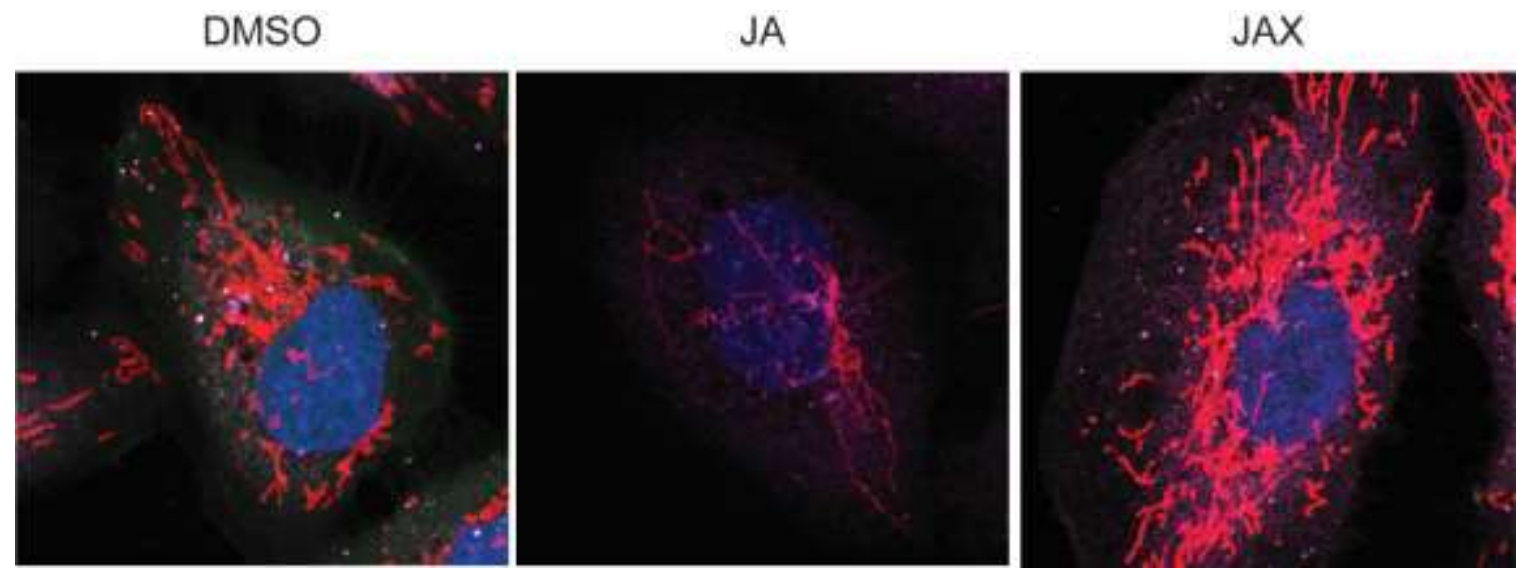

Figure 4-1. Treatment with Jatrophone but not Jatrogossone A causes severe alterations in mitochondrial morphology.

U2OS cells were treated with DMSO, $1.25 \mu \mathrm{M}$ Ja, or $1.25 \mu \mathrm{M}$ JAX for 8 hours. Cells were the fixed and stained with DAPI (blue) and antibodies for TOMM20 (red), p62 (purple), and LC3 (green). 
Because of the observed induction of ROS, I next interrogated whether Jatrogossone A could induce mitophagy events. Mitophagy was measured by using U2OS cells expressing mitochondrial targeted mKeima. Keima is activated by the $440 \mathrm{~nm}$ light at neutral $\mathrm{pH}$ (purple) but in the acidic conditions of the lysosome Keima is activated by $560 \mathrm{~nm}$ light (green), allowing us to distinguish between cytoplasmic mitochondria and mitochondria in the last stages of mitophagic degradation. Treatment of cells with either Jatrophone or Jatrogossone A at $1.25 \mu \mathrm{M}$ and $2.5 \mu \mathrm{M}$ for $8 \mathrm{hr}$ induced mitophagy (Figure 4-2). However, Jatrogossone A led to higher mitophagy induction at $2.5 \mu \mathrm{M}$ as shown in the relative quantification. Thus, this data strongly suggests that Jatrogossone A can induce mitophagy and correlate with ROS induction in cancer cell models. Importantly, the mitophagy-inducing concentration is at least five-fold lower than the concentration required to induce apoptosis. Hence, this compound is a promising chemical probe to study mitophagy in cancer
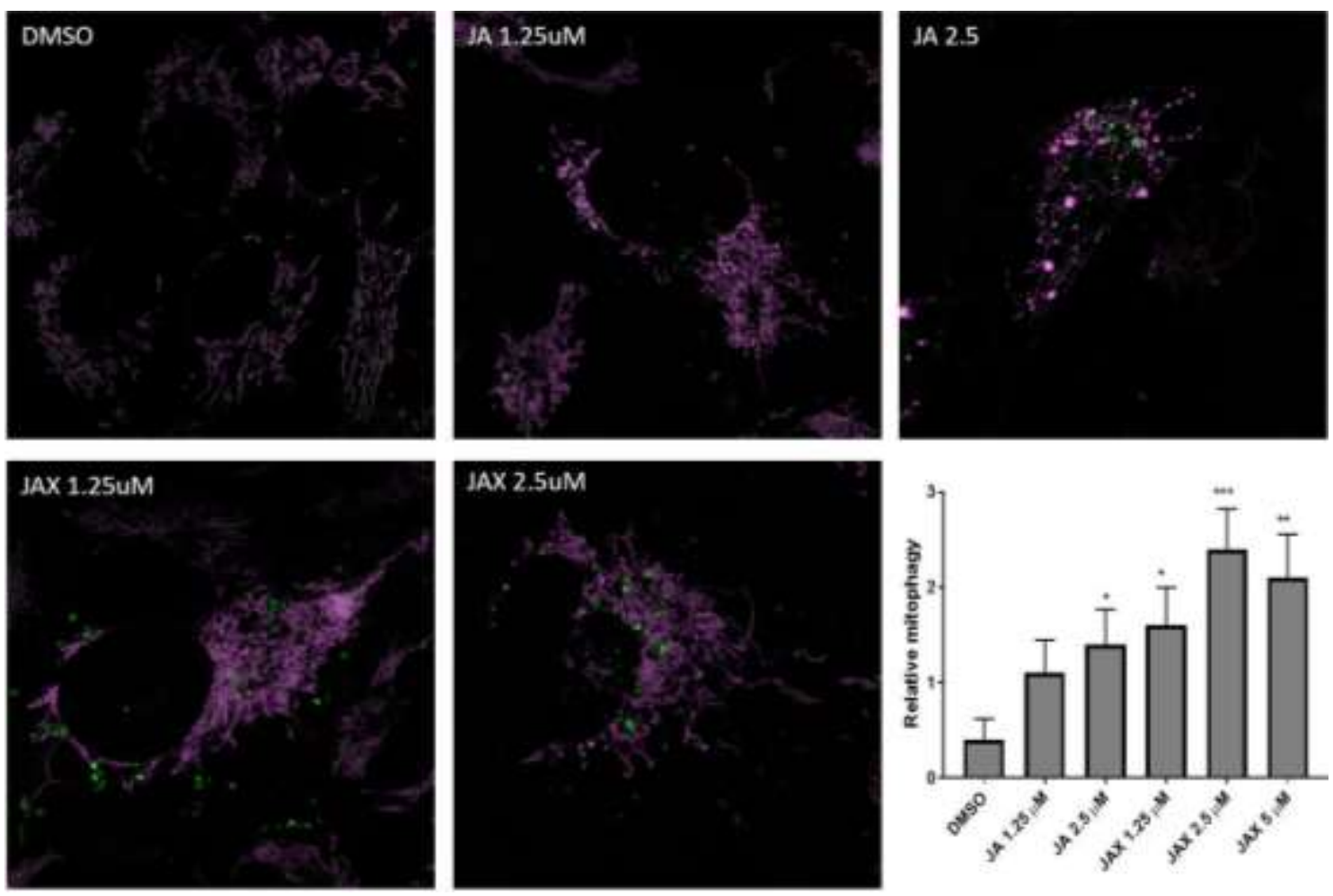

Figure 4-2. Treatment with Jatrogossone A induces mitophagy.

U2OS cells stably expressing mito-mKeima were treated with DMSO, $1.25 \mu \mathrm{M} \mathrm{Ja}, 2.5 \mu \mathrm{M}$ Ja, $1.25 \mu \mathrm{M}$ JAX, or $2.5 \mu \mathrm{M}$ JAX for 8 hours. Relative mitophagy levels were quantified and normalized.

Adapted with permission from Ling T, Lang WH, Craig J, et al. Studies of Jatrogossone A as a Reactive Oxygen Species Inducer in Cancer Cellular Models. J Nat Prod. 2019;82(5):1301-1311. https://doi.org/10.1021/acs.jnatprod.8b01087. Copyright (2019) American Chemical Society. 


\section{CHAPTER 5. DISCUSSION}

\section{Conclusions}

Currently, there is substantial interest in the role autophagy plays in maintaining normal physiology and how dysfunctional mitophagy can lead to or enhance diseases. Our discovery of the MEKK3-MEK5-ERK5 pathway as a novel mitophagy regulator could facilitate a deeper understanding of how different mechanisms act to maintain homeostasis and prevent disease in different tissues. Here, we show that basal mitochondrial degradation requires p62 and MEKK3-MEK5-ERK5 signaling and is quite distinct from damage-induced mitophagy, which does not require p62 or MEKK3MEK5-ERK5. One of the key findings of this dissertation is the importance of p62 in mitophagy under basal conditions. We have found that $\mathrm{p} 62$ routinely delivers mitochondria to lysosomes without the addition of exogenous damage. We also found that inhibition of autophagy non-specifically with Bafilomycin A1 increases total mitochondrial load in cells, indicating that mitophagy is an ongoing maintenance process.

Using genetic and pharmacological inhibition of the MEKK3-MEK5-ERK5 pathway, we observed mitochondrial content increase in human cancer cells, mouse embryonic fibroblasts, and primary erythroid progenitor cells isolated from the murine fetal liver. This suggests that MEKK3-MEK5-ERK5 restrains mitochondrial accumulation in diverse cells and tissues. We also observed an increase in the levels of mitophagy receptor protein p62 after pharmacological or genetic inhibition of MEKK3MEK5-ERK5 signaling. I confirmed that inhibition of pathway activity results in decreased lysosome-dependent degradation of mitochondria using mitochondrial targeted mKeima, without affecting lysosome acidification or non-selective bulk-autophagy levels in the cell. Taken together, it was found that the MEKK3-MEK5-ERK5 pathway promotes mitophagy without the addition of exogenous mitochondrial damage, which could have great implication on studying mitophagy in the context of development and disease.

We also sought to investigate whether developmentally programmed mitochondrial degradation in the erythrocyte lineage required MEK5-ERK5 signaling as a well-established model of mitochondrial clearance in the absence of exogenous damage. Although impaired maturation of erythrocytes upon MEK5 or ERK5 inhibition precluded full assessment of the impact on mitochondrial turnover, the mitochondrial content was increased at each stage of erythroid maturation in MEK5-ERK5 inhibited cells. Taken together with the lack of a requirement for MEKK3-MEK5-ERK5 signaling in CCCP-induced mitophagy, it appears that the MEKK3-MEK5-ERK5 pathway may be solely designated for basal mitochondrial clearance. It is also possible that MEKK3MEK5-ERK5 signaling may be required for induction of mitochondrial degradation by other stimuli, such as statin treatment, hypoxia, or forced metabolic switch from glycolytic metabolism to oxidative phosphorylation (Andres et al., 2014; L. Liu et al., 2012; Sin et al., 2016; Wilkinson, Sidaway, \& Cross, 2018). These questions remain to be addressed in future work. 


\section{Future Directions}

Whether MEKK3-MEK5-ERK5 signaling controls mitochondrial degradation by directly or indirectly regulating p62 remains to be determined, although we have ruled out the possibility that MEKK3-MEK5-ERK5 signaling is required for p62 expression or stability. Direct interactions between the PB1 domain of p62 and the PB1 domains of MEKK3 and MEK5 have been reported, providing a potential means for direct regulation of p62 by the MEKK3-MEK5-ERK5 kinase cascade (Lamark et al., 2003; Nakamura et al., 2010).

While MEKK3-MEK5-ERK5 signaling has been implicated in many aspects of mammalian development and disease, it is also the most newly discovered MAP kinase pathway, and much work needs to be done to fully understand how the MEKK3-MEK5ERK5 kinase cascade is acting. It will be interesting to determine whether any of these previously identified roles for the ERK5 pathway in development, physiology, or disease represent an underlying role for mitochondrial degradation. For example, ERK5 signaling is well established to play an anti-apoptotic role, and mitochondria harbor several proapoptotic molecules including cytochrome $c$, Smac/DIABLO, and AIF (Adrain, Creagh, \& Martin, 2001; Baechler, Bloemberg, \& Quadrilatero, 2019; Nithianandarajah-Jones et al., 2012). It is possible that active ERK5 signaling antagonizes autophagy in part through elimination of pro-apoptotic molecules through delivery of mitochondria to lysosomes for degradation. These and other interesting hypotheses arising from our current work remain to be tested in future studies.

Additionally, we helped discover a novel mitophagy inducing drug, Jatrogossone A. Recently, there has been interest in using pharmacological screens to identify novel chemical modulators that might be used to promote the efficient removal of damaged mitochondria in hopes of restoring the cell to metabolic homeostasis. Mitochondrial uncouplers, like CCCP, have been widely used to induce mitochondrial removal via the activation of the PINK1/Parkin pathway (Geisler et al., 2010; Narendra et al., 2008; Okatsu et al., 2010). However, we have shown that CCCP does not effectively induce all mitophagy pathways, therefore, it is useful to have several tools that could be used to stimulate different mechanisms of mitophagy. Next, we could expand these techniques to create a larger scale screening process to search for new natural products to induce mitophagy. 


\section{LIST OF REFERENCES}

Adrain, C., Creagh, E. M., \& Martin, S. J. (2001). Apoptosis-associated release of Smac/DIABLO from mitochondria requires active caspases and is blocked by Bcl-2. Embo Journal, 20(23), 6627-6636. https://doi.org/10.1093/emboj/20.23.6627

Aerbajinai, W., Giattina, M., Lee, Y. T., Raffeld, M., \& Miller, J. L. (2003). The proapoptotic factor Nix is coexpressed with Bcl-xL during terminal erythroid differentiation. Blood, 102(2), 712-717. https://doi.org/10.1182/blood-2002-113324

Airavaara, M., Parkkinen, I., Konovalova, J., Albert, K., Chmielarz, P., \& Domanskyi, A. (2020). Back and to the Future: From Neurotoxin-Induced to Human Parkinson's Disease Models. Curr Protoc Neurosci, 91(1), e88. https://doi.org/10.1002/cpns.88

Andres, A. M., Hernandez, G., Lee, P., Huang, C., Ratliff, E. P., Sin, J., . . . Gottlieb, R. A. (2014). Mitophagy is required for acute cardioprotection by simvastatin. Antioxid Redox Signal, 21(14), 1960-1973. https://doi.org/10.1089/ars.2013.5416

Angulo-Ibanez, M., Rovira-Clave, X., Granados-Jaen, A., Downs, B., Kim, Y. C., Wang, S. M., . . Espel, E. (2015). Erk5 contributes to maintaining the balance of cellular nucleotide levels and erythropoiesis. Cell Cycle, 14(24), 3864-3876. https://doi.org/10.1080/15384101.2015.1120914

Ashford, T. P., \& Porter, K. R. (1962). Cytoplasmic components in hepatic cell lysosomes. J Cell Biol, 12, 198-202. https://doi.org/10.1083/jcb.12.1.198

Baechler, B. L., Bloemberg, D., \& Quadrilatero, J. (2019). Mitophagy regulates mitochondrial network signaling, oxidative stress, and apoptosis during myoblast differentiation. Autophagy, 15(9), 1606-1619. https://doi.org/10.1080/15548627.2019.1591672

Biel, T. G., \& Rao, V. A. (2018). Mitochondrial dysfunction activates lysosomaldependent mitophagy selectively in cancer cells. Oncotarget, 9(1), 995-1011. https://doi.org/10.18632/oncotarget.23171

Billia, F., Hauck, L., Konecny, F., Rao, V., Shen, J., \& Mak, T. W. (2011). PTENinducible kinase 1 (PINK1)/Park6 is indispensable for normal heart function. Proc Natl Acad Sci U S A, 108(23), 9572-9577. https://doi.org/10.1073/pnas.1106291108

Blesa, J., \& Przedborski, S. (2014). Parkinson's disease: animal models and dopaminergic cell vulnerability. Front Neuroanat, 8, 155. https://doi.org/10.3389/fnana.2014.00155

Bueno, C. A., Michelini, F. M., Pertino, M. W., Gomez, C. A., Schmeda-Hirschmann, G., \& Alche, L. E. (2015). Natural and semisynthetic diterpenoids with antiviral and immunomodulatory activities block the ERK signaling pathway. Med Microbiol Immunol, 204(5), 575-584. https://doi.org/10.1007/s00430-014-0383-9

Chen, G., Han, Z., Feng, D., Chen, Y., Chen, L., Wu, H., . . Chen, Q. (2014). A regulatory signaling loop comprising the PGAM5 phosphatase and CK2 controls receptor-mediated mitophagy. Mol Cell, 54(3), 362-377. https://doi.org/10.1016/j.molcel.2014.02.034 
Clark, S. L., Jr. (1957). Cellular differentiation in the kidneys of newborn mice studies with the electron microscope. J Biophys Biochem Cytol, 3(3), 349-362. https://doi.org/10.1083/jcb.3.3.349

Connelly, J. P., \& Pruett-Miller, S. M. (2019). CRIS.py: A Versatile and High-throughput Analysis Program for CRISPR-based Genome Editing. Sci Rep, 9(1), 4194. https://doi.org/10.1038/s41598-019-40896-w

Cuervo, A. M., \& Wong, E. (2014). Chaperone-mediated autophagy: roles in disease and aging. Cell Res, 24(1), 92-104. https://doi.org/10.1038/cr.2013.153

Cullere, X., Plovie, E., Bennett, P. M., MacRae, C. A., \& Mayadas, T. N. (2015). The cerebral cavernous malformation proteins CCM2L and CCM2 prevent the activation of the MAP kinase MEKK3. Proc Natl Acad Sci U S A, 112(46), 14284-14289. https://doi.org/10.1073/pnas.1510495112

De Duve, C., Pressman, B. C., Gianetto, R., Wattiaux, R., \& Appelmans, F. (1955). Tissue fractionation studies. 6. Intracellular distribution patterns of enzymes in rat-liver tissue. Biochem J, 60(4), 604-617. https://doi.org/10.1042/bj0600604

Denison, S. R., Wang, F., Becker, N. A., Schule, B., Kock, N., Phillips, L. A., . . Smith, D. I. (2003). Alterations in the common fragile site gene Parkin in ovarian and other cancers. Oncogene, 22(51), 8370-8378. https://doi.org/10.1038/sj.onc.1207072

Dikic, I. (2017). Proteasomal and Autophagic Degradation Systems. Annu Rev Biochem, 86, 193-224. https://doi.org/10.1146/annurev-biochem-061516-044908

Dikic, I., \& Elazar, Z. (2018). Mechanism and medical implications of mammalian autophagy. Nat Rev Mol Cell Biol, 19(6), 349-364. https://doi.org/10.1038/s41580-018-0003-4

Ding, W. X., \& Yin, X. M. (2012). Mitophagy: mechanisms, pathophysiological roles, and analysis. Biol Chem, 393(7), 547-564. https://doi.org/10.1515/hsz-2012-0119

Drake, L. E., Springer, M. Z., Poole, L. P., Kim, C. J., \& Macleod, K. F. (2017). Expanding perspectives on the significance of mitophagy in cancer. Semin Cancer Biol, 47, 110-124. https://doi.org/10.1016/j.semcancer.2017.04.008

Drew, B. A., Burow, M. E., \& Beckman, B. S. (2012). MEK5/ERK5 pathway: the first fifteen years. Biochim Biophys Acta, 1825(1), 37-48. https://doi.org/10.1016/j.bbcan.2011.10.002

Elmore, S. P., Qian, T., Grissom, S. F., \& Lemasters, J. J. (2001). The mitochondrial permeability transition initiates autophagy in rat hepatocytes. FASEB J, 15(12), 2286-2287. https://doi.org/10.1096/fj.01-0206fje

Esteban-Martinez, L., \& Boya, P. (2018). BNIP3L/NIX-dependent mitophagy regulates cell differentiation via metabolic reprogramming. Autophagy, 14(5), 915-917. https://doi.org/10.1080/15548627.2017.1332567

Esteban-Martinez, L., Sierra-Filardi, E., McGreal, R. S., Salazar-Roa, M., Marino, G., Seco, E., . . Boya, P. (2017). Programmed mitophagy is essential for the glycolytic switch during cell differentiation. EMBO J, 36(12), 1688-1706. https://doi.org/10.15252/embj.201695916

Farre, J. C., \& Subramani, S. (2016). Mechanistic insights into selective autophagy pathways: lessons from yeast. Nat Rev Mol Cell Biol, 17(9), 537-552. https://doi.org/10.1038/nrm.2016.74 
Filomeni, G., De Zio, D., \& Cecconi, F. (2015). Oxidative stress and autophagy: the clash between damage and metabolic needs. Cell Death Differ, 22(3), 377-388. https://doi.org/10.1038/cdd.2014.150

Fujiwara, M., Marusawa, H., Wang, H. Q., Iwai, A., Ikeuchi, K., Imai, Y., . . Chiba, T. (2008). Parkin as a tumor suppressor gene for hepatocellular carcinoma. Oncogene, 27(46), 6002-6011. https://doi.org/10.1038/onc.2008.199

Gao, F., Chen, D., Si, J., Hu, Q., Qin, Z., Fang, M., \& Wang, G. (2015). The mitochondrial protein BNIP3L is the substrate of PARK2 and mediates mitophagy in PINK1/PARK2 pathway. Hum Mol Genet, 24(9), 2528-2538. https://doi.org/10.1093/hmg/ddv017

Gao, F., Yang, J., Wang, D., Li, C., Fu, Y., Wang, H., . . Z Zhang, J. (2017). Mitophagy in Parkinson's Disease: Pathogenic and Therapeutic Implications. Front Neurol, 8, 527. https://doi.org/10.3389/fneur.2017.00527

Geisler, S., Holmstrom, K. M., Skujat, D., Fiesel, F. C., Rothfuss, O. C., Kahle, P. J., \& Springer, W. (2010). PINK1/Parkin-mediated mitophagy is dependent on VDAC1 and p62/SQSTM1. Nat Cell Biol, 12(2), 119-131. https://doi.org/10.1038/ncb2012

Gleyzer, N., Vercauteren, K., \& Scarpulla, R. C. (2005). Control of mitochondrial transcription specificity factors (TFB1M and TFB2M) by nuclear respiratory factors (NRF-1 and NRF-2) and PGC-1 family coactivators. Mol Cell Biol, 25(4), 1354-1366. https://doi.org/10.1128/MCB.25.4.1354-1366.2005

Glick, D., Barth, S., \& Macleod, K. F. (2010). Autophagy: cellular and molecular mechanisms. J Pathol, 221(1), 3-12. https://doi.org/10.1002/path.2697

Gomez, N., Erazo, T., \& Lizcano, J. M. (2016). ERK5 and Cell Proliferation: Nuclear Localization Is What Matters. Front Cell Dev Biol, 4, 105. https://doi.org/10.3389/fcell.2016.00105

Greene, J. C., Whitworth, A. J., Kuo, I., Andrews, L. A., Feany, M. B., \& Pallanck, L. J. (2003). Mitochondrial pathology and apoptotic muscle degeneration in Drosophila parkin mutants. Proc Natl Acad Sci U S A, 100(7), 4078-4083. https://doi.org/10.1073/pnas.0737556100

Gustafsson, A. B., \& Dorn, G. W., 2nd. (2019). Evolving and Expanding the Roles of Mitophagy as a Homeostatic and Pathogenic Process. Physiol Rev, 99(1), 853892. https://doi.org/10.1152/physrev.00005.2018

Hadi, V., Hotard, M., Ling, T., Salinas, Y. G., Palacios, G., Connelly, M., \& Rivas, F. (2013). Evaluation of Jatropha isabelli natural products and their synthetic analogs as potential antimalarial therapeutic agents. Eur J Med Chem, 65, 376380. https://doi.org/10.1016/j.ejmech.2013.04.030

Hahn, A., \& Zuryn, S. (2019). Mitochondrial Genome (mtDNA) Mutations that Generate Reactive Oxygen Species. Antioxidants (Basel), 8(9). https://doi.org/10.3390/antiox8090392

Hamacher-Brady, A., Brady, N. R., Logue, S. E., Sayen, M. R., Jinno, M., Kirshenbaum, L. A., .. . Gustafsson, A. B. (2007). Response to myocardial ischemia/reperfusion injury involves Bnip3 and autophagy. Cell Death Differ, 14(1), 146-157. https://doi.org/10.1038/sj.cdd.4401936

Harvey, A. L., Edrada-Ebel, R., \& Quinn, R. J. (2015). The re-emergence of natural products for drug discovery in the genomics era. Nat Rev Drug Discov, 14(2), 111-129. https://doi.org/10.1038/nrd4510 
Hayashi, M., Kim, S. W., Imanaka-Yoshida, K., Yoshida, T., Abel, E. D., Eliceiri, B., . . . Lee, J. D. (2004). Targeted deletion of BMK1/ERK5 in adult mice perturbs vascular integrity and leads to endothelial failure. J Clin Invest, 113(8), 11381148. https://doi.org/10.1172/JCI19890

Honda, S., Arakawa, S., Nishida, Y., Yamaguchi, H., Ishii, E., \& Shimizu, S. (2014). Ulk1-mediated Atg5-independent macroautophagy mediates elimination of mitochondria from embryonic reticulocytes. Nat Commun, 5, 4004. https://doi.org/10.1038/ncomms5004

Hosokawa, N., Hara, T., Kaizuka, T., Kishi, C., Takamura, A., Miura, Y., . . Mizushima, N. (2009). Nutrient-dependent mTORC1 association with the ULK1-Atg13FIP200 complex required for autophagy. Mol Biol Cell, 20(7), 1981-1991. https://doi.org/10.1091/mbc.E08-12-1248

Hu, C., Fan, L., Cen, P., Chen, E., Jiang, Z., \& Li, L. (2016). Energy Metabolism Plays a Critical Role in Stem Cell Maintenance and Differentiation. Int J Mol Sci, 17(2), 253. https://doi.org/10.3390/ijms17020253

Huynh, D. P., Dy, M., Nguyen, D., Kiehl, T. R., \& Pulst, S. M. (2001). Differential expression and tissue distribution of parkin isoforms during mouse development. Brain Res Dev Brain Res, 130(2), 173-181.

Ikeda, Y., Shirakabe, A., Maejima, Y., Zhai, P., Sciarretta, S., Toli, J., . . Sadoshima, J. (2015). Endogenous Drp1 mediates mitochondrial autophagy and protects the heart against energy stress. Circ Res, 116(2), 264-278. https://doi.org/10.1161/CIRCRESAHA.116.303356

Jin, S. M., Lazarou, M., Wang, C., Kane, L. A., Narendra, D. P., \& Youle, R. J. (2010). Mitochondrial membrane potential regulates PINK1 import and proteolytic destabilization by PARL. J Cell Biol, 191(5), 933-942. https://doi.org/10.1083/jcb.201008084

Jornayvaz, F. R., \& Shulman, G. I. (2010). Regulation of mitochondrial biogenesis. Essays Biochem, 47, 69-84. https://doi.org/10.1042/bse0470069

Kanki, T., Wang, K., Cao, Y., Baba, M., \& Klionsky, D. J. (2009). Atg32 is a mitochondrial protein that confers selectivity during mitophagy. Dev Cell, 17(1), 98-109. https://doi.org/10.1016/j.devcel.2009.06.014

Kausar, S., Wang, F., \& Cui, H. (2018). The Role of Mitochondria in Reactive Oxygen Species Generation and Its Implications for Neurodegenerative Diseases. Cells, 7(12). https://doi.org/10.3390/cells7120274

Khan, A. U. H., Allende-Vega, N., Gitenay, D., Garaude, J., Vo, D. N., Belkhala, S., . . . Villalba, M. (2018). Mitochondrial Complex I activity signals antioxidant response through ERK5. Sci Rep, 8(1), 7420. https://doi.org/10.1038/s41598-01823884-4

Khandia, R., Dadar, M., Munjal, A., Dhama, K., Karthik, K., Tiwari, R., . . . Chaicumpa, W. (2019). A Comprehensive Review of Autophagy and Its Various Roles in Infectious, Non-Infectious, and Lifestyle Diseases: Current Knowledge and Prospects for Disease Prevention, Novel Drug Design, and Therapy. Cells, 8(7). https://doi.org/10.3390/cells8070674

Kim, N. C., Tresse, E., Kolaitis, R. M., Molliex, A., Thomas, R. E., Alami, N. H., . . Taylor, J. P. (2013). VCP is essential for mitochondrial quality control by 
PINK1/Parkin and this function is impaired by VCP mutations. Neuron, 78(1), 65-80. https://doi.org/10.1016/j.neuron.2013.02.029

Kirkin, V. (2020). History of the Selective Autophagy Research: How Did It Begin and Where Does It Stand Today? J Mol Biol, 432(1), 3-27. https://doi.org/10.1016/j.jmb.2019.05.010

Kogure, T., Kawano, H., Abe, Y., \& Miyawaki, A. (2008). Fluorescence imaging using a fluorescent protein with a large Stokes shift. Methods, 45(3), 223-226. https://doi.org/10.1016/j.ymeth.2008.06.009

Koulnis, M., Pop, R., Porpiglia, E., Shearstone, J. R., Hidalgo, D., \& Socolovsky, M. (2011). Identification and analysis of mouse erythroid progenitors using the CD71/TER119 flow-cytometric assay. J Vis $\operatorname{Exp}(54)$. https://doi.org/10.3791/2809

Kubli, D. A., Zhang, X., Lee, Y., Hanna, R. A., Quinsay, M. N., Nguyen, C. K., . . . Gustafsson, A. B. (2013). Parkin protein deficiency exacerbates cardiac injury and reduces survival following myocardial infarction. J Biol Chem, 288(2), 915-926. https://doi.org/10.1074/jbc.M112.411363

Kundu, M., Lindsten, T., Yang, C. Y., Wu, J., Zhao, F., Zhang, J., . . Thompson, C. B. (2008). Ulk1 plays a critical role in the autophagic clearance of mitochondria and ribosomes during reticulocyte maturation. Blood, 112(4), 1493-1502. https://doi.org/10.1182/blood-2008-02-137398

Lamark, T., Perander, M., Outzen, H., Kristiansen, K., Overvatn, A., Michaelsen, E., .. . Johansen, T. (2003). Interaction codes within the family of mammalian Phox and Bem1p domain-containing proteins. J Biol Chem, 278(36), 34568-34581. https://doi.org/10.1074/jbc.M303221200

Lazarou, M., Jin, S. M., Kane, L. A., \& Youle, R. J. (2012). Role of PINK1 binding to the TOM complex and alternate intracellular membranes in recruitment and activation of the E3 ligase Parkin. Dev Cell, 22(2), 320-333. https://doi.org/10.1016/j.devcel.2011.12.014

Lazarou, M., Sliter, D. A., Kane, L. A., Sarraf, S. A., Wang, C., Burman, J. L., . . Youle, R. J. (2015). The ubiquitin kinase PINK1 recruits autophagy receptors to induce mitophagy. Nature, 524(7565), 309-314. https://doi.org/10.1038/nature14893

Lee, J. D., Ulevitch, R. J., \& Han, J. (1995). Primary structure of BMK1: a new mammalian map kinase. Biochem Biophys Res Commun, 213(2), 715-724. https://doi.org/10.1006/bbrc.1995.2189

Lee, Y., Lee, H. Y., Hanna, R. A., \& Gustafsson, A. B. (2011). Mitochondrial autophagy by Bnip3 involves Drp1-mediated mitochondrial fission and recruitment of Parkin in cardiac myocytes. Am J Physiol Heart Circ Physiol, 301(5), H1924-1931. https://doi.org/10.1152/ajpheart.00368.2011

Ling, T., Lang, W. H., Craig, J., Potts, M. B., Budhraja, A., Opferman, J., . . Rivas, F. (2019). Studies of Jatrogossone A as a Reactive Oxygen Species Inducer in Cancer Cellular Models. J Nat Prod, 82(5), 1301-1311. https://doi.org/10.1021/acs.jnatprod.8b01087

Liu, L., Feng, D., Chen, G., Chen, M., Zheng, Q., Song, P., . . Chen, Q. (2012). Mitochondrial outer-membrane protein FUNDC1 mediates hypoxia-induced mitophagy in mammalian cells. Nat Cell Biol, 14(2), 177-185. https://doi.org/10.1038/ncb2422 
Liu, W., Ruiz-Velasco, A., Wang, S., Khan, S., Zi, M., Jungmann, A., . . Wang, X. (2017). Metabolic stress-induced cardiomyopathy is caused by mitochondrial dysfunction due to attenuated Erk5 signaling. Nat Commun, 8(1), 494. https://doi.org/10.1038/s41467-017-00664-8

Luo, S. M., Ge, Z. J., Wang, Z. W., Jiang, Z. Z., Wang, Z. B., Ouyang, Y. C., . . Sun, Q. Y. (2013). Unique insights into maternal mitochondrial inheritance in mice. Proc Natl Acad Sci U S A, 110(32), 13038-13043. https://doi.org/10.1073/pnas.1303231110

Matsumoto, G., Shimogori, T., Hattori, N., \& Nukina, N. (2015). TBK1 controls autophagosomal engulfment of polyubiquitinated mitochondria through p62/SQSTM1 phosphorylation. Hum Mol Genet. https://doi.org/10.1093/hmg/ddv179

Mattiazzi, M., Vijayvergiya, C., Gajewski, C. D., DeVivo, D. C., Lenaz, G., Wiedmann, M., \& Manfredi, G. (2004). The mtDNA T8993G (NARP) mutation results in an impairment of oxidative phosphorylation that can be improved by antioxidants. Hum Mol Genet, 13(8), 869-879. https://doi.org/10.1093/hmg/ddh103

McArthur, K., Whitehead, L. W., Heddleston, J. M., Li, L., Padman, B. S., Oorschot, V., ... Kile, B. T. (2018). BAK/BAX macropores facilitate mitochondrial herniation and mtDNA efflux during apoptosis. Science, 359(6378). https://doi.org/10.1126/science.aao6047

McWilliams, T. G., Prescott, A. R., Montava-Garriga, L., Ball, G., Singh, F., Barini, E., . . . Ganley, I. G. (2018). Basal Mitophagy Occurs Independently of PINK1 in Mouse Tissues of High Metabolic Demand. Cell Metab, 27(2), 439-449 e435. https://doi.org/10.1016/j.cmet.2017.12.008

Mijaljica, D., Prescott, M., \& Devenish, R. J. (2011). Microautophagy in mammalian cells: revisiting a 40-year-old conundrum. Autophagy, 7(7), 673-682. https://doi.org/10.4161/auto.7.7.14733

Mizushima, N. (2005). The pleiotropic role of autophagy: from protein metabolism to bactericide. Cell Death Differ, 12 Suppl 2, 1535-1541. https://doi.org/10.1038/sj.cdd.4401728

Mizushima, N. (2007). Autophagy: process and function. Genes Dev, 21(22), 2861-2873. https://doi.org/10.1101/gad.1599207

Moras, M., Lefevre, S. D., \& Ostuni, M. A. (2017). From Erythroblasts to Mature Red Blood Cells: Organelle Clearance in Mammals. Front Physiol, 8, 1076. https://doi.org/10.3389/fphys.2017.01076

Mortensen, M., Ferguson, D. J., Edelmann, M., Kessler, B., Morten, K. J., Komatsu, M., $\&$ Simon, A. K. (2010). Loss of autophagy in erythroid cells leads to defective removal of mitochondria and severe anemia in vivo. Proc Natl Acad Sci U S A, 107(2), 832-837. https://doi.org/10.1073/pnas.0913170107

Murphy, M. P. (2009). How mitochondria produce reactive oxygen species. Biochem J, 417(1), 1-13. https://doi.org/10.1042/BJ20081386

Nakamura, K., Kimple, A. J., Siderovski, D. P., \& Johnson, G. L. (2010). PB1 domain interaction of p62/sequestosome 1 and MEKK3 regulates NF-kappaB activation. $J$ Biol Chem, 285(3), 2077-2089. https://doi.org/10.1074/jbc.M109.065102 
Nakatogawa, H., Suzuki, K., Kamada, Y., \& Ohsumi, Y. (2009). Dynamics and diversity in autophagy mechanisms: lessons from yeast. Nat Rev Mol Cell Biol, 10(7), 458467. https://doi.org/10.1038/nrm2708

Narendra, D., Kane, L. A., Hauser, D. N., Fearnley, I. M., \& Youle, R. J. (2010). p62/SQSTM1 is required for Parkin-induced mitochondrial clustering but not mitophagy; VDAC1 is dispensable for both. Autophagy, 6(8), 1090-1106. https://doi.org/10.4161/auto.6.8.13426

Narendra, D., Tanaka, A., Suen, D. F., \& Youle, R. J. (2008). Parkin is recruited selectively to impaired mitochondria and promotes their autophagy. J Cell Biol, 183(5), 795-803. https://doi.org/10.1083/jcb.200809125

Nguyen, T. D., Shaid, S., Vakhrusheva, O., Koschade, S. E., Klann, K., Tholken, M., . . . Brandts, C. H. (2019). Loss of the selective autophagy receptor p62 impairs murine myeloid leukemia progression and mitophagy. Blood, 133(2), 168-179. https://doi.org/10.1182/blood-2018-02-833475

Nguyen, T. N., Padman, B. S., Usher, J., Oorschot, V., Ramm, G., \& Lazarou, M. (2016). Atg8 family LC3/GABARAP proteins are crucial for autophagosome-lysosome fusion but not autophagosome formation during PINK1/Parkin mitophagy and starvation. J Cell Biol, 215(6), 857-874. https://doi.org/10.1083/jcb.201607039

Nishimoto, S., \& Nishida, E. (2006). MAPK signalling: ERK5 versus ERK1/2. EMBO Rep, 7(8), 782-786. https://doi.org/10.1038/sj.embor.7400755

Nissanka, N., \& Moraes, C. T. (2018). Mitochondrial DNA damage and reactive oxygen species in neurodegenerative disease. FEBS Lett, 592(5), 728-742. https://doi.org/10.1002/1873-3468.12956

Nithianandarajah-Jones, G. N., Wilm, B., Goldring, C. E., Muller, J., \& Cross, M. J. (2012). ERK5: structure, regulation and function. Cell Signal, 24(11), 2187-2196. https://doi.org/10.1016/i.cellsig.2012.07.007

Novak, I., Kirkin, V., McEwan, D. G., Zhang, J., Wild, P., Rozenknop, A., . . Dikic, I. (2010). Nix is a selective autophagy receptor for mitochondrial clearance. EMBO Rep, 11(1), 45-51. https://doi.org/10.1038/embor.2009.256

Okamoto, K., Kondo-Okamoto, N., \& Ohsumi, Y. (2009). Mitochondria-anchored receptor Atg32 mediates degradation of mitochondria via selective autophagy. Dev Cell, 17(1), 87-97. https://doi.org/10.1016/j.devcel.2009.06.013

Okatsu, K., Saisho, K., Shimanuki, M., Nakada, K., Shitara, H., Sou, Y. S., . . Matsuda, N. (2010). p62/SQSTM1 cooperates with Parkin for perinuclear clustering of depolarized mitochondria. Genes Cells, 15(8), 887-900.

https://doi.org/10.1111/j.1365-2443.2010.01426.x

Okatsu, K., Uno, M., Koyano, F., Go, E., Kimura, M., Oka, T., . . Matsuda, N. (2013). A dimeric PINK1-containing complex on depolarized mitochondria stimulates Parkin recruitment. J Biol Chem, 288(51), 36372-36384. https://doi.org/10.1074/ibc.M113.509653

Palikaras, K., Lionaki, E., \& Tavernarakis, N. (2018). Mechanisms of mitophagy in cellular homeostasis, physiology and pathology. Nat Cell Biol, 20(9), 1013-1022. https://doi.org/10.1038/s41556-018-0176-2

Pankiv, S., Clausen, T. H., Lamark, T., Brech, A., Bruun, J. A., Outzen, H., . . Johansen, T. (2007). p62/SQSTM1 binds directly to Atg8/LC3 to facilitate degradation of 
ubiquitinated protein aggregates by autophagy. J Biol Chem, 282(33), 2413124145. https://doi.org/10.1074/jbc.M702824200

Pickles, S., Vigie, P., \& Youle, R. J. (2018). Mitophagy and Quality Control Mechanisms in Mitochondrial Maintenance. Curr Biol, 28(4), R170-R185. https://doi.org/10.1016/j.cub.2018.01.004

Pickrell, A. M., Huang, C. H., Kennedy, S. R., Ordureau, A., Sideris, D. P., Hoekstra, J. G., . . Y Youle, R. J. (2015). Endogenous Parkin Preserves Dopaminergic Substantia Nigral Neurons following Mitochondrial DNA Mutagenic Stress. Neuron, 87(2), 371-381. https://doi.org/10.1016/j.neuron.2015.06.034

Potts, M. B., Kim, H. S., Fisher, K. W., Hu, Y., Carrasco, Y. P., Bulut, G. B., . . White, M. A. (2013). Using functional signature ontology (FUSION) to identify mechanisms of action for natural products. Sci Signal, 6(297), ra90. https://doi.org/10.1126/scisignal.2004657

Richter, B., Sliter, D. A., Herhaus, L., Stolz, A., Wang, C., Beli, P., . . Dikic, I. (2016). Phosphorylation of OPTN by TBK1 enhances its binding to Ub chains and promotes selective autophagy of damaged mitochondria. Proc Natl Acad Sci U S A, 113(15), 4039-4044. https://doi.org/10.1073/pnas.1523926113

Rojansky, R., Cha, M. Y., \& Chan, D. C. (2016). Elimination of paternal mitochondria in mouse embryos occurs through autophagic degradation dependent on PARKIN and MUL1. Elife, 5. https://doi.org/10.7554/eLife.17896

Scarpulla, R. C. (2011). Metabolic control of mitochondrial biogenesis through the PGC1 family regulatory network. Biochim Biophys Acta, 1813(7), 1269-1278. https://doi.org/10.1016/j.bbamcr.2010.09.019

Schweers, R. L., Zhang, J., Randall, M. S., Loyd, M. R., Li, W., Dorsey, F. C., . . Ney, P. A. (2007). NIX is required for programmed mitochondrial clearance during reticulocyte maturation. Proc Natl Acad Sci U S A, 104(49), 19500-19505. https://doi.org/10.1073/pnas.0708818104

Shah, S. P., Roth, A., Goya, R., Oloumi, A., Ha, G., Zhao, Y., . . A Aparicio, S. (2012). The clonal and mutational evolution spectrum of primary triple-negative breast cancers. Nature, 486(7403), 395-399. https://doi.org/10.1038/nature10933

Sharpley, M. S., Marciniak, C., Eckel-Mahan, K., McManus, M., Crimi, M., Waymire, K., . . W Wallace, D. C. (2012). Heteroplasmy of mouse mtDNA is genetically unstable and results in altered behavior and cognition. Cell, 151(2), 333-343. https://doi.org/10.1016/j.cell.2012.09.004

Shi, Q. W., Su, X. H., \& Kiyota, H. (2008). Chemical and pharmacological research of the plants in genus Euphorbia. Chem Rev, 108(10), 4295-4327. https://doi.org/10.1021/cr078350s

Shiba-Fukushima, K., Imai, Y., Yoshida, S., Ishihama, Y., Kanao, T., Sato, S., \& Hattori, N. (2012). PINK1-mediated phosphorylation of the Parkin ubiquitin-like domain primes mitochondrial translocation of Parkin and regulates mitophagy. Sci Rep, 2, 1002. https://doi.org/10.1038/srep01002

Simoes, A. E., Rodrigues, C. M., \& Borralho, P. M. (2016). The MEK5/ERK5 signalling pathway in cancer: a promising novel therapeutic target. Drug Discov Today, 21(10), 1654-1663. https://doi.org/10.1016/j.drudis.2016.06.010

Sin, J., Andres, A. M., Taylor, D. J., Weston, T., Hiraumi, Y., Stotland, A., . . Gottlieb, R. A. (2016). Mitophagy is required for mitochondrial biogenesis and myogenic 
differentiation of C2C12 myoblasts. Autophagy, 12(2), 369-380.

https://doi.org/10.1080/15548627.2015.1115172

Sliter, D. A., Martinez, J., Hao, L., Chen, X., Sun, N., Fischer, T. D., . . Youle, R. J. (2018). Parkin and PINK1 mitigate STING-induced inflammation. Nature, 561(7722), 258-262. https://doi.org/10.1038/s41586-018-0448-9

Song, W. H., Yi, Y. J., Sutovsky, M., Meyers, S., \& Sutovsky, P. (2016). Autophagy and ubiquitin-proteasome system contribute to sperm mitophagy after mammalian fertilization. Proc Natl Acad Sci U S A, 113(36), E5261-5270. https://doi.org/10.1073/pnas.1605844113

Stolz, A., Ernst, A., \& Dikic, I. (2014). Cargo recognition and trafficking in selective autophagy. Nat Cell Biol, 16(6), 495-501. https://doi.org/10.1038/ncb2979

Sulahian, R., Cleaver, O., \& Huang, L. J. (2009). Ligand-induced EpoR internalization is mediated by JAK 2 and $\mathrm{p} 85$ and is impaired by mutations responsible for primary familial and congenital polycythemia. Blood, 113(21), 5287-5297. https://doi.org/10.1182/blood-2008-09-179572

Sutovsky, P., Moreno, R. D., Ramalho-Santos, J., Dominko, T., Simerly, C., \& Schatten, G. (2000). Ubiquitinated sperm mitochondria, selective proteolysis, and the regulation of mitochondrial inheritance in mammalian embryos. Biol Reprod, 63(2), 582-590. https://doi.org/10.1095/biolreprod63.2.582

Tantama, M., Hung, Y. P., \& Yellen, G. (2011). Imaging intracellular pH in live cells

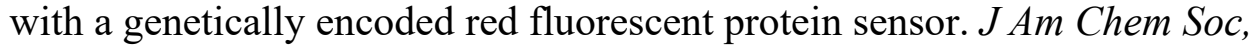
133(26), 10034-10037. https://doi.org/10.1021/ja202902d

Tatake, R. J., O'Neill, M. M., Kennedy, C. A., Wayne, A. L., Jakes, S., Wu, D., . . . Snow, R. J. (2008). Identification of pharmacological inhibitors of the MEK5/ERK5 pathway. Biochem Biophys Res Commun, 377(1), 120-125. https://doi.org/10.1016/j.bbrc.2008.09.087

Trifunovic, A., Wredenberg, A., Falkenberg, M., Spelbrink, J. N., Rovio, A. T., Bruder, C. E., . . Larsson, N. G. (2004). Premature ageing in mice expressing defective mitochondrial DNA polymerase. Nature, 429(6990), 417-423. https://doi.org/10.1038/nature02517

Tsukada, M., \& Ohsumi, Y. (1993). Isolation and characterization of autophagy-defective mutants of Saccharomyces cerevisiae. FEBS Lett, 333(1-2), 169-174. https://doi.org/10.1016/0014-5793(93)80398-e

Tubita, A., Lombardi, Z., Tusa, I., Dello Sbarba, P., \& Rovida, E. (2020). Beyond Kinase Activity: ERK5 Nucleo-Cytoplasmic Shuttling as a Novel Target for Anticancer Therapy. Int J Mol Sci, 2l(3). https://doi.org/10.3390/ijms21030938

Vazquez-Martin, A., Van den Haute, C., Cufi, S., Corominas-Faja, B., Cuyas, E., LopezBonet, E., . . Menendez, J. A. (2016). Mitophagy-driven mitochondrial rejuvenation regulates stem cell fate. Aging (Albany NY), 8(7), 1330-1352. https://doi.org/10.18632/aging.100976

Veeriah, S., Taylor, B. S., Meng, S., Fang, F., Yilmaz, E., Vivanco, I., . . Chan, T. A. (2010). Somatic mutations of the Parkinson's disease-associated gene PARK2 in glioblastoma and other human malignancies. Nat Genet, 42(1), 77-82. https://doi.org/10.1038/ng.491 
Villarroya, J., Giralt, M., \& Villarroya, F. (2009). Mitochondrial DNA: an up-andcoming actor in white adipose tissue pathophysiology. Obesity (Silver Spring), 17(10), 1814-1820. https://doi.org/10.1038/oby.2009.152

Wei, Y., Chiang, W. C., Sumpter, R., Jr., Mishra, P., \& Levine, B. (2017). Prohibitin 2 Is an Inner Mitochondrial Membrane Mitophagy Receptor. Cell, 168(1-2), 224-238 e210. https://doi.org/10.1016/j.cell.2016.11.042

Wen, X., \& Klionsky, D. J. (2016). An overview of macroautophagy in yeast. J Mol Biol, 428(9 Pt A), 1681-1699. https://doi.org/10.1016/j.jmb.2016.02.021

West, A. P., Khoury-Hanold, W., Staron, M., Tal, M. C., Pineda, C. M., Lang, S. M., . . . Shadel, G. S. (2015). Mitochondrial DNA stress primes the antiviral innate immune response. Nature, 520(7548), 553-557.

https://doi.org/10.1038/nature14156

Wilkinson, E. L., Sidaway, J. E., \& Cross, M. J. (2018). Statin regulated ERK5 stimulates tight junction formation and reduces permeability in human cardiac endothelial cells. J Cell Physiol, 233(1), 186-200. https://doi.org/10.1002/jcp.26064

Xiao, Y., Zhou, Y., Lu, Y., Zhou, K., \& Cai, W. (2018). PHB2 interacts with LC3 and SQSTM1 is required for bile acids-induced mitophagy in cholestatic liver. Cell Death Dis, 9(2), 160. https://doi.org/10.1038/s41419-017-0228-8

Yamada, T., Dawson, T. M., Yanagawa, T., Iijima, M., \& Sesaki, H. (2019). SQSTM1/p62 promotes mitochondrial ubiquitination independently of PINK1 and PRKN/parkin in mitophagy. Autophagy, 1-7. https://doi.org/10.1080/15548627.2019.1643185

Yamada, T., Murata, D., Adachi, Y., Itoh, K., Kameoka, S., Igarashi, A., . . Sesaki, H. (2018). Mitochondrial Stasis Reveals p62-Mediated Ubiquitination in ParkinIndependent Mitophagy and Mitigates Nonalcoholic Fatty Liver Disease. Cell Metab. https://doi.org/10.1016/i.cmet.2018.06.014

Yang, Q., Deng, X., Lu, B., Cameron, M., Fearns, C., Patricelli, M. P., . . Lee, J. D. (2010). Pharmacological inhibition of BMK1 suppresses tumor growth through promyelocytic leukemia protein. Cancer Cell, 18(3), 258-267. https://doi.org/10.1016/j.ccr.2010.08.008

Yang, Y., Gehrke, S., Imai, Y., Huang, Z., Ouyang, Y., Wang, J. W., . . . Lu, B. (2006). Mitochondrial pathology and muscle and dopaminergic neuron degeneration caused by inactivation of Drosophila Pink1 is rescued by Parkin. Proc Natl Acad Sci U S A, 103(28), 10793-10798. https://doi.org/10.1073/pnas.0602493103

Yoo, S. M., \& Jung, Y. K. (2018). A Molecular Approach to Mitophagy and Mitochondrial Dynamics. Mol Cells, 41(1), 18-26. https://doi.org/10.14348/molcells.2018.2277

Zaffagnini, G., \& Martens, S. (2016). Mechanisms of Selective Autophagy. J Mol Biol, 428(9 Pt A), 1714-1724. https://doi.org/10.1016/j.jmb.2016.02.004

Zhang, C., Lin, M., Wu, R., Wang, X., Yang, B., Levine, A. J., . . Feng, Z. (2011). Parkin, a p53 target gene, mediates the role of p53 in glucose metabolism and the Warburg effect. Proc Natl Acad Sci U S A, 108(39), 16259-16264. https://doi.org/10.1073/pnas.1113884108

Zhang, T., Xue, L., Li, L., Tang, C., Wan, Z., Wang, R., . . . Zhang, Z. (2016). BNIP3 Protein Suppresses PINK1 Kinase Proteolytic Cleavage to Promote Mitophagy. $J$ Biol Chem, 291(41), 21616-21629. https://doi.org/10.1074/jbc.M116.733410 
Zhou, G., Bao, Z. Q., \& Dixon, J. E. (1995). Components of a new human protein kinase signal transduction pathway. J Biol Chem, 270(21), 12665-12669. https://doi.org/10.1074/jbc.270.21.12665

Zhou, T., Chen, C., Xu, C., Zhou, H., Gao, B., Su, D., . . Su, P. (2018). Mutant MAPK7Induced Idiopathic Scoliosis is Linked to Impaired Osteogenesis. Cell Physiol Biochem, 48(3), 880-890. https://doi.org/10.1159/000491956

Zhu, H., Guariglia, S., Li, W., Brancho, D., Wang, Z. V., Scherer, P. E., \& Chow, C. W. (2014). Role of extracellular signal-regulated kinase 5 in adipocyte signaling. $J$ Biol Chem, 289(9), 6311-6322. https://doi.org/10.1074/jbc.M113.506584 
APPENDIX. BIX02188 AND BIX02189 KINOME PROFILING

\begin{tabular}{|c|c|c|c|c|}
\hline \multirow[b]{2}{*}{ Kinase } & \multicolumn{2}{|c|}{ BIX02188 } & \multicolumn{2}{|c|}{ BIX02189 } \\
\hline & $\begin{array}{c}0.0043 \\
\mu \mathrm{M}\end{array}$ & $\begin{array}{c}0.043 \\
\mu \mathrm{M}\end{array}$ & $\begin{array}{c}0.0015 \\
\mu \mathrm{M}\end{array}$ & $\begin{array}{c}0.015 \\
\mu \mathrm{M}\end{array}$ \\
\hline Abl (H396P) (h) & 110 & 96 & 109 & 109 \\
\hline Abl (M351T)(h) & 96 & 96 & 105 & 102 \\
\hline Abl (Q252H) (h) & 98 & 93 & 96 & 107 \\
\hline $\mathrm{Abl}(\mathrm{h})$ & 102 & 113 & 105 & 103 \\
\hline $\operatorname{Abl}(\mathrm{m})$ & 93 & 99 & 103 & 102 \\
\hline Abl(T315I)(h) & 97 & 99 & 100 & 99 \\
\hline $\mathrm{Abl}(\mathrm{Y} 253 \mathrm{~F})(\mathrm{h})$ & 97 & 97 & 109 & 105 \\
\hline ACK1(h) & 95 & 104 & 109 & 97 \\
\hline $\operatorname{ALK}(\mathrm{h})$ & 106 & 114 & 106 & 105 \\
\hline ALK4(h) & 97 & 104 & 108 & 99 \\
\hline AMPK $\alpha 1(\mathrm{~h})$ & 111 & 118 & 109 & 1 \\
\hline AMPK $\alpha 2(\mathrm{~h})$ & 101 & 106 & 105 & 113 \\
\hline $\operatorname{Arg}(\mathrm{h})$ & 110 & 107 & 118 & 116 \\
\hline $\operatorname{Arg}(\mathrm{m})$ & 104 & 99 & 101 & 100 \\
\hline ARK5(h) & 96 & 102 & 107 & 92 \\
\hline ASK1(h) & 123 & 97 & 134 & 129 \\
\hline Aurora-A(h) & 115 & 107 & 117 & 111 \\
\hline Aurora-B(h) & 112 & 101 & 113 & 123 \\
\hline Aurora-C(h) & 114 & 103 & 106 & 92 \\
\hline $\operatorname{Axl}(\mathrm{h})$ & 113 & 116 & 113 & 111 \\
\hline Blk(h) & 104 & 99 & 111 & 105 \\
\hline $\mathrm{Blk}(\mathrm{m})$ & 90 & 102 & 110 & 113 \\
\hline $\operatorname{Bmx}(\mathrm{h})$ & 105 & 104 & 102 & 98 \\
\hline $\mathrm{BRK}(\mathrm{h})$ & 111 & 108 & 110 & 113 \\
\hline BrSK1(h) & 110 & 109 & 102 & 95 \\
\hline BrSK2(h) & 100 & 108 & 107 & 105 \\
\hline $\mathrm{BTK}(\mathrm{h})$ & 111 & 96 & 110 & 102 \\
\hline BTK $(\mathrm{R} 28 \mathrm{H})(\mathrm{h})$ & 101 & 103 & 89 & 86 \\
\hline CaMKI(h) & 114 & 110 & 102 & 96 \\
\hline CaMKII $\beta(h)$ & 112 & 103 & 109 & 111 \\
\hline CaMKII $\gamma(\mathrm{h})$ & 102 & 98 & 100 & 96 \\
\hline CaMKIIS(h) & 112 & 115 & 113 & 96 \\
\hline CaMKIV(h) & 109 & 116 & 100 & 108 \\
\hline CaMKI $\delta(\mathrm{h})$ & 109 & 108 & 109 & 114 \\
\hline CDK1/cyclinB(h) & 99 & 106 & 96 & 94 \\
\hline CDK2/cyclinA(h) & 100 & 101 & 103 & 99 \\
\hline CDK2/cyclinE(h) & 95 & 99 & 92 & 95 \\
\hline CDK3/cyclinE(h) & 111 & 111 & 115 & 95 \\
\hline CDK5/p25(h) & 103 & 101 & 102 & 103 \\
\hline CDK5/p35(h) & 101 & 99 & 106 & 98 \\
\hline
\end{tabular}




\begin{tabular}{|c|c|c|c|c|}
\hline CDK6/cyclinD3(h) & 105 & 110 & 127 & 105 \\
\hline CDK7/cyclinH/MAT1(h) & 100 & 97 & 101 & 97 \\
\hline CDK9/cyclin T1(h) & 102 & 101 & 101 & 112 \\
\hline CHK1(h) & 99 & 100 & 106 & 103 \\
\hline CHK2(h) & 98 & 100 & 111 & 108 \\
\hline CHK2(I157T)(h) & 115 & 109 & 121 & 109 \\
\hline CHK2(R145W)(h) & 109 & 97 & 106 & 98 \\
\hline CK1(y) & 105 & 96 & 99 & 101 \\
\hline CK $1 \gamma 1(\mathrm{~h})$ & 104 & 102 & 110 & 108 \\
\hline CK1 $1 \gamma 2(h)$ & 107 & 105 & 95 & 98 \\
\hline CK $1 \gamma 3(h)$ & 91 & 105 & 102 & 105 \\
\hline $\mathrm{CK} 1 \delta(\mathrm{h})$ & 106 & 103 & 100 & 105 \\
\hline CK2(h) & 102 & 105 & 104 & 98 \\
\hline $\mathrm{CK} 2 \alpha 2(\mathrm{~h})$ & 100 & 98 & 97 & 94 \\
\hline cKit(D816H)(h) & 81 & 31 & 93 & 79 \\
\hline $\operatorname{cKit}(\mathrm{D} 816 \mathrm{~V})(\mathrm{h})$ & 104 & 96 & 104 & 100 \\
\hline cKit(h) & 104 & 100 & 113 & 106 \\
\hline cKit(V560G)(h) & 86 & 34 & 113 & 92 \\
\hline cKit(V654A)(h) & 85 & 53 & 94 & 87 \\
\hline CLK2(h) & 95 & 92 & 98 & 99 \\
\hline CLK3(h) & 104 & 109 & 108 & 103 \\
\hline $\mathrm{c}-\mathrm{RAF}(\mathrm{h})$ & 102 & 98 & 102 & 105 \\
\hline $\operatorname{CSK}(\mathrm{h})$ & 102 & 102 & 97 & 106 \\
\hline $\operatorname{cSRC}(\mathrm{h})$ & 111 & 98 & 110 & 99 \\
\hline DAPK1(h) & 105 & 110 & 110 & 99 \\
\hline DAPK2(h) & 107 & 104 & 112 & 102 \\
\hline DCAMKL2(h) & 124 & 111 & 114 & 102 \\
\hline DDR2(h) & 127 & 121 & 120 & 118 \\
\hline DMPK(h) & 107 & 114 & 119 & 102 \\
\hline DRAK1(h) & 100 & 109 & 111 & 117 \\
\hline DYRK2(h) & 94 & 107 & 102 & 107 \\
\hline eEF-2K(h) & 102 & 104 & 98 & 99 \\
\hline EGFR(h) & 115 & 105 & 101 & 102 \\
\hline EGFR(L858R)(h) & 109 & 104 & 104 & 98 \\
\hline EGFR(L861Q)(h) & 105 & 94 & 103 & 107 \\
\hline EGFR(T790M)(h) & 104 & 109 & 107 & 96 \\
\hline EGFR(T790M,L858R)(h) & 94 & 98 & 105 & 98 \\
\hline $\operatorname{EphA1(h)}$ & 108 & 104 & 110 & 107 \\
\hline EphA2(h) & 127 & 100 & 117 & 119 \\
\hline EphA3(h) & 96 & 108 & 113 & 103 \\
\hline EphA4(h) & 110 & 107 & 110 & 102 \\
\hline EphA5(h) & 98 & 95 & 99 & 105 \\
\hline EphA7(h) & 103 & 104 & 101 & 99 \\
\hline EphA8(h) & 102 & 107 & 107 & 102 \\
\hline EphB1(h) & 117 & 100 & 107 & 105 \\
\hline EphB2(h) & 98 & 105 & 94 & 91 \\
\hline
\end{tabular}




\begin{tabular}{|c|c|c|c|c|}
\hline EphB3(h) & 104 & 100 & 103 & 99 \\
\hline EphB4(h) & 98 & 100 & 107 & 104 \\
\hline ErbB4(h) & 114 & 112 & 123 & 110 \\
\hline FAK(h) & 102 & 103 & 101 & 98 \\
\hline $\operatorname{Fer}(\mathrm{h})$ & 112 & 104 & 112 & 110 \\
\hline Fes(h) & 85 & 99 & 110 & 10 \\
\hline FGFR1(h) & 112 & 108 & 108 & 10 \\
\hline FGFR1(V561M)(h) & 106 & 98 & 97 & 101 \\
\hline FGFR2(h) & 103 & 102 & 97 & 98 \\
\hline FGFR2(N549H)(h) & 105 & 102 & 109 & 103 \\
\hline FGFR3(h) & 107 & 101 & 107 & 109 \\
\hline FGFR4(h) & 105 & 110 & 101 & 102 \\
\hline $\operatorname{Fgr}(h)$ & 94 & 105 & 106 & 99 \\
\hline Flt1(h) & 110 & 109 & 118 & 102 \\
\hline Flt3(D835Y)(h) & 105 & 105 & 96 & 10 \\
\hline Flt3(h) & 84 & 88 & 93 & 107 \\
\hline Flt4(h) & 106 & 101 & 108 & 94 \\
\hline $\operatorname{Fms}(\mathrm{h})$ & 106 & 100 & 102 & 97 \\
\hline Fms(Y969C)(h) & 100 & 78 & 101 & 84 \\
\hline Fyn(h) & 88 & 64 & 104 & 93 \\
\hline GCK(h) & 124 & 100 & 102 & 99 \\
\hline GRK5(h) & 99 & 103 & 103 & 97 \\
\hline GRK6(h) & 97 & 104 & 102 & 100 \\
\hline GRK7(h) & 95 & 93 & 114 & 9 \\
\hline GSK3 $3 \alpha(h)$ & 92 & 93 & 95 & 95 \\
\hline GSK3 $\beta(h)$ & 94 & 88 & 86 & 91 \\
\hline Haspin(h) & 106 & 92 & 102 & 100 \\
\hline $\operatorname{Hck}(\mathrm{h})$ & 96 & 74 & 101 & 87 \\
\hline $\operatorname{Hck}(\mathrm{h})$ activated & 100 & 82 & 102 & 105 \\
\hline HIPK1(h) & 115 & 114 & 112 & 102 \\
\hline HIPK2(h) & 112 & 105 & 105 & 112 \\
\hline HIPK3(h) & 101 & 108 & 105 & 10 \\
\hline IGF-1R(h) & 102 & 95 & 100 & 99 \\
\hline IGF-1R(h), activated & 110 & 115 & 113 & 101 \\
\hline $\operatorname{IKK} \alpha(\mathrm{h})$ & 113 & 106 & 104 & 113 \\
\hline $\operatorname{IKK} \beta(\mathrm{h})$ & 111 & 109 & 101 & 104 \\
\hline $\operatorname{IR}(\mathrm{h})$ & 101 & 104 & 104 & 9 \\
\hline $\operatorname{IR}(\mathrm{h})$, activated & 107 & 110 & 111 & 10 \\
\hline IRAK1(h) & 106 & 103 & 102 & 11 \\
\hline IRAK4(h) & 95 & 110 & 100 & 11 \\
\hline $\operatorname{IRR}(\mathrm{h})$ & 112 & 132 & 120 & 11 \\
\hline $\operatorname{Itk}(\mathrm{h})$ & 99 & 94 & 107 & 9 \\
\hline JAK2(h) & 112 & 114 & 97 & 111 \\
\hline JAK3(h) & 101 & 106 & 103 & 97 \\
\hline JNK $1 \alpha 1(\mathrm{~h})$ & 108 & 103 & 95 & 97 \\
\hline JNK $2 \alpha 2(\mathrm{~h})$ & 109 & 110 & 99 & 102 \\
\hline
\end{tabular}




\begin{tabular}{|c|c|c|c|c|}
\hline JNK3(h) & 102 & 107 & 107 & 100 \\
\hline $\mathrm{KDR}(\mathrm{h})$ & 100 & 102 & 103 & 113 \\
\hline $\operatorname{Lck}(\mathrm{h})$ & 100 & 85 & 103 & 89 \\
\hline Lck(h) activated & 106 & 97 & 111 & 103 \\
\hline LIMK1(h) & 102 & 103 & 107 & 97 \\
\hline LKB1(h) & 101 & 96 & 104 & 104 \\
\hline $\mathrm{LOK}(\mathrm{h})$ & 98 & 94 & 109 & 96 \\
\hline Lyn(h) & 95 & 100 & 99 & 92 \\
\hline $\operatorname{Lyn}(m)$ & 93 & 95 & 93 & 98 \\
\hline MAPK1(h) & 101 & 106 & 106 & 102 \\
\hline MAPK2(h) & 105 & 105 & 101 & 96 \\
\hline MAPK2(m) & 108 & 117 & 114 & 106 \\
\hline MAPKAP-K2(h) & 99 & 93 & 102 & 100 \\
\hline MAPKAP-K3(h) & 99 & 94 & 100 & 93 \\
\hline MARK1(h) & 105 & 105 & 110 & 113 \\
\hline MEK1(h) & 118 & 123 & 117 & 121 \\
\hline $\operatorname{MELK}(\mathrm{h})$ & 93 & 77 & 85 & 82 \\
\hline $\operatorname{Mer}(\mathrm{h})$ & 110 & 109 & 102 & 102 \\
\hline $\operatorname{Met}(\mathrm{D} 1246 \mathrm{H})(\mathrm{h})$ & 108 & 100 & 98 & 102 \\
\hline $\operatorname{Met}(\mathrm{D} 1246 \mathrm{~N})(\mathrm{h})$ & 92 & 100 & 100 & 99 \\
\hline $\operatorname{Met}(\mathrm{h})$ & 122 & 133 & 107 & 144 \\
\hline $\operatorname{Met}(\mathrm{M} 1268 \mathrm{~T})(\mathrm{h})$ & 99 & 115 & 101 & 109 \\
\hline $\operatorname{Met}(Y 1248 C)(h)$ & 105 & 98 & 111 & 100 \\
\hline $\operatorname{Met}(Y 1248 D)(h)$ & 99 & 97 & 107 & 107 \\
\hline $\operatorname{Met}(Y 1248 H)(h)$ & 95 & 93 & 102 & 101 \\
\hline MINK(h) & 110 & 110 & 106 & 112 \\
\hline MKK4(m) & 122 & 103 & 126 & 114 \\
\hline MKK6(h) & 92 & 71 & 91 & 94 \\
\hline MKK $7 \beta(h)$ & 137 & 137 & 132 & 128 \\
\hline $\operatorname{MLCK}(\mathrm{h})$ & 99 & 106 & 98 & 94 \\
\hline MLK1(h) & 96 & 104 & 107 & 93 \\
\hline Mnk2(h) & 104 & 101 & 97 & 105 \\
\hline $\operatorname{MRCK} \alpha(\mathrm{h})$ & 113 & 115 & 106 & 104 \\
\hline $\operatorname{MRCK} \beta(h)$ & 99 & 108 & 102 & 105 \\
\hline MSK1(h) & 108 & 106 & 105 & 103 \\
\hline MSK2(h) & 125 & 117 & 116 & 120 \\
\hline MSSK1(h) & 105 & 110 & 99 & 95 \\
\hline MST1(h) & 107 & 103 & 109 & 100 \\
\hline MST2(h) & 104 & 117 & 101 & 106 \\
\hline MST3(h) & 88 & 109 & 95 & 99 \\
\hline mTOR(h) & 104 & 95 & 101 & 95 \\
\hline mTOR/FKBP12(h) & 92 & 105 & 97 & 90 \\
\hline $\operatorname{MuSK}(\mathrm{h})$ & 88 & 109 & 95 & 82 \\
\hline NEK11(h) & 103 & 111 & 100 & 95 \\
\hline NEK2(h) & 100 & 91 & 99 & 94 \\
\hline NEK3(h) & 111 & 107 & 125 & 125 \\
\hline
\end{tabular}




\begin{tabular}{|c|c|c|c|c|}
\hline NEK6(h) & 103 & 112 & 101 & 100 \\
\hline NEK7(h) & 119 & 112 & 111 & 110 \\
\hline NLK(h) & 98 & 97 & 105 & 96 \\
\hline p70S6K(h) & 101 & 110 & 104 & 118 \\
\hline PAK2(h) & 96 & 109 & 96 & 88 \\
\hline PAK4(h) & 97 & 103 & 97 & 103 \\
\hline PAK5(h) & 128 & 121 & 113 & 119 \\
\hline PAK6(h) & 102 & 104 & 103 & 104 \\
\hline PAR-1B $\alpha(h)$ & 105 & 105 & 104 & 103 \\
\hline $\operatorname{PASK}(\mathrm{h})$ & 105 & 105 & 92 & 105 \\
\hline PDGFR $\alpha(\mathrm{D} 842 \mathrm{~V})(\mathrm{h})$ & 96 & 73 & 98 & 88 \\
\hline $\operatorname{PDGFR} \alpha(\mathrm{h})$ & 104 & 97 & 88 & 108 \\
\hline PDGFR $\alpha(V 561 D)(h)$ & 94 & 55 & 101 & 103 \\
\hline PDGFR $\beta(h)$ & 114 & 101 & 127 & 130 \\
\hline PDK1(h) & 106 & 111 & 105 & 103 \\
\hline $\operatorname{PEK}(\mathrm{h})$ & 101 & 102 & 100 & 100 \\
\hline $\mathrm{PhK} \gamma 2(\mathrm{~h})$ & 102 & 110 & 117 & 113 \\
\hline PI3 Kinase & & & & \\
\hline $\begin{array}{c}(\mathrm{p} 110 \mathrm{a}(\mathrm{E} 542 \mathrm{~K}) / \mathrm{p} 85 \mathrm{a})(\mathrm{h}) \\
\text { PI3 Kinase }\end{array}$ & 103 & 102 & 102 & 103 \\
\hline $\begin{array}{c}(\mathrm{p} 110 \mathrm{a}(\mathrm{E} 542 \mathrm{~K}) / \mathrm{p} 85 \mathrm{a})(\mathrm{m}) \\
\text { PI3 Kinase }\end{array}$ & 102 & 101 & 103 & 103 \\
\hline $\begin{array}{c}(\mathrm{p} 110 \mathrm{a}(\mathrm{E} 545 \mathrm{~K}) / \mathrm{p} 85 \mathrm{a})(\mathrm{h}) \\
\text { PI3 Kinase }\end{array}$ & 99 & 102 & 100 & 61 \\
\hline $\begin{array}{c}(\mathrm{p} 110 \mathrm{a}(\mathrm{E} 545 \mathrm{~K}) / \mathrm{p} 85 \mathrm{a})(\mathrm{m}) \\
\text { PI3 Kinase }\end{array}$ & 99 & 100 & 98 & 97 \\
\hline $\begin{array}{c}(\mathrm{p} 110 \mathrm{a}(\mathrm{H} 1047 \mathrm{R}) / \mathrm{p} 85 \mathrm{a})(\mathrm{h}) \\
\text { PI3 Kinase }\end{array}$ & 104 & 102 & 102 & 101 \\
\hline$(\mathrm{p} 110 \mathrm{a}(\mathrm{H} 1047 \mathrm{R}) / \mathrm{p} 85 \mathrm{a})(\mathrm{m})$ & 101 & 100 & 102 & 101 \\
\hline PI3 Kinase (p110a/p65a)(h) & 103 & 101 & 101 & 99 \\
\hline PI3 Kinase (p110a/p65a)(m) & 98 & 99 & 97 & 99 \\
\hline PI3 Kinase (p110a/p85a)(h) & 101 & 99 & 99 & 101 \\
\hline PI3 Kinase (p110a/p85a)(m) & 101 & 105 & 99 & 104 \\
\hline PI3 Kinase (p110b/p85a)(h) & 97 & 96 & 105 & 101 \\
\hline PI3 Kinase (p110b/p85a)(m) & 100 & 100 & 105 & 101 \\
\hline PI3 Kinase (p110b/p85b)(m) & 103 & 101 & 102 & 107 \\
\hline PI3 Kinase $(p 110 d / p 85 a)(h)$ & 102 & 104 & 102 & 102 \\
\hline PI3 Kinase $(p 110 d / p 85 a)(m)$ & 106 & 104 & 103 & 100 \\
\hline PI3 Kinase $(p 120 g)(h)$ & 100 & 92 & 101 & 107 \\
\hline $\mathrm{PI} 3 \mathrm{KC} 2 \mathrm{a}(\mathrm{h})$ & 103 & 96 & 98 & 103 \\
\hline PI3KC2g(h) & 99 & 97 & 98 & 100 \\
\hline Pim-1(h) & 109 & 101 & 106 & 104 \\
\hline Pim-2(h) & 94 & 93 & 110 & 103 \\
\hline Pim-3(h) & 99 & 104 & 99 & 97 \\
\hline PIP4K $2 a(h)$ & 107 & 104 & 98 & 101 \\
\hline PIP5K1a(h) & 103 & 98 & 100 & 100 \\
\hline
\end{tabular}




\begin{tabular}{|c|c|c|c|c|}
\hline PIP5K1g(h) & 96 & 90 & 98 & 93 \\
\hline PKA(h) & 108 & 109 & 101 & 104 \\
\hline $\mathrm{PKB} \alpha(\mathrm{h})$ & 104 & 107 & 107 & 108 \\
\hline $\mathrm{PKB} \beta(\mathrm{h})$ & 106 & 98 & 107 & 107 \\
\hline $\mathrm{PKB} \gamma(\mathrm{h})$ & 117 & 116 & 104 & 107 \\
\hline $\mathrm{PKC} \alpha(\mathrm{h})$ & 103 & 102 & 101 & 105 \\
\hline PKC $\beta I(h)$ & 104 & 114 & 110 & 119 \\
\hline PKC $\beta I I(h)$ & 107 & 107 & 101 & 101 \\
\hline $\mathrm{PKC} \gamma(\mathrm{h})$ & 100 & 102 & 100 & 88 \\
\hline $\operatorname{PKC} \delta(\mathrm{h})$ & 109 & 97 & 110 & 105 \\
\hline $\operatorname{PKC} \varepsilon(\mathrm{h})$ & 103 & 103 & 102 & 113 \\
\hline $\mathrm{PKC} \zeta(\mathrm{h})$ & 110 & 100 & 106 & 104 \\
\hline $\mathrm{PKC \eta}(\mathrm{h})$ & 110 & 109 & 96 & 98 \\
\hline $\operatorname{PKC} \theta(\mathrm{h})$ & 109 & 107 & 112 & 107 \\
\hline $\mathrm{PKCl}(\mathrm{h})$ & 103 & 101 & 94 & 109 \\
\hline $\mathrm{PKC} \mu(\mathrm{h})$ & 103 & 109 & 111 & 105 \\
\hline PKD2(h) & 101 & 102 & 116 & 117 \\
\hline PKG1 $\alpha(h)$ & 107 & 93 & 110 & 108 \\
\hline PKG1 $\beta(h)$ & 109 & 113 & 100 & 120 \\
\hline Plk1(h) & 106 & 115 & 110 & 107 \\
\hline Plk3(h) & 97 & 95 & 93 & 94 \\
\hline PRAK(h) & 97 & 101 & 100 & 90 \\
\hline PRK2(h) & 108 & 105 & 113 & 107 \\
\hline $\operatorname{PrKX}(\mathrm{h})$ & 120 & 126 & 122 & 120 \\
\hline PTK5(h) & 108 & 122 & 103 & 99 \\
\hline Pyk2(h) & 96 & 99 & 105 & 99 \\
\hline Ret (V804L)(h) & 99 & 90 & 103 & 96 \\
\hline $\operatorname{Ret}(\mathrm{h})$ & 101 & 114 & 109 & 98 \\
\hline $\operatorname{Ret}(\mathrm{V} 804 \mathrm{M})(\mathrm{h})$ & 102 & 101 & 106 & 106 \\
\hline RIPK2(h) & 97 & 103 & 96 & 95 \\
\hline ROCK-I(h) & 94 & 104 & 108 & 105 \\
\hline ROCK-II(h) & 110 & 112 & 109 & 105 \\
\hline ROCK-II(r) & 105 & 107 & 115 & 110 \\
\hline Ron(h) & 103 & 115 & 112 & 102 \\
\hline $\operatorname{Ros}(h)$ & 110 & 106 & 104 & 99 \\
\hline Rse(h) & 101 & 104 & 110 & 97 \\
\hline Rsk1(h) & 98 & 106 & 101 & 98 \\
\hline Rsk1(r) & 107 & 101 & 109 & 115 \\
\hline Rsk2(h) & 95 & 100 & 109 & 107 \\
\hline Rsk3(h) & 110 & 108 & 113 & 108 \\
\hline Rsk4(h) & 108 & 110 & 108 & 104 \\
\hline SAPK2a(h) & 109 & 105 & 112 & 107 \\
\hline SAPK2a(T106M)(h) & 95 & 97 & 106 & 103 \\
\hline SAPK2b(h) & 109 & 96 & 95 & 105 \\
\hline SAPK3(h) & 99 & 103 & 109 & 102 \\
\hline SAPK4(h) & 123 & 110 & 120 & 120 \\
\hline
\end{tabular}




\begin{tabular}{|c|c|c|c|c|}
\hline SGK(h) & 96 & 99 & 93 & 101 \\
\hline SGK2(h) & 92 & 100 & 98 & 108 \\
\hline SGK3(h) & 111 & 122 & 108 & 110 \\
\hline $\operatorname{SIK}(\mathrm{h})$ & 108 & 102 & 102 & 104 \\
\hline $\operatorname{Snk}(\mathrm{h})$ & 116 & 113 & 121 & 109 \\
\hline $\operatorname{Src}(1-530)(\mathrm{h})$ & 97 & 91 & 90 & 99 \\
\hline $\operatorname{Src}(\mathrm{T} 341 \mathrm{M})(\mathrm{h})$ & 97 & 106 & 101 & 98 \\
\hline SRPK1(h) & 107 & 109 & 103 & 104 \\
\hline SRPK2(h) & 105 & 105 & 105 & 106 \\
\hline STK33(h) & 106 & 110 & 103 & 104 \\
\hline Syk(h) & 100 & 101 & 112 & 107 \\
\hline TAK1(h) & 104 & 105 & 109 & 101 \\
\hline TAO1(h) & 104 & 112 & 108 & 103 \\
\hline TAO2(h) & 119 & 97 & 120 & 106 \\
\hline TAO3(h) & 109 & 106 & 112 & 100 \\
\hline TBK1(h) & 109 & 111 & 102 & 103 \\
\hline Tec $(\mathrm{h})$ activated & 113 & 102 & 94 & 104 \\
\hline TGFBR1(h) & 103 & 100 & 106 & 101 \\
\hline Tie2 (h) & 93 & 101 & 96 & 105 \\
\hline Tie2(R849W)(h) & 94 & 104 & 97 & 99 \\
\hline Tie2(Y897S)(h) & 93 & 96 & 94 & 93 \\
\hline TLK2(h) & 106 & 104 & 109 & 100 \\
\hline $\operatorname{TrkA}(\mathrm{h})$ & 112 & 105 & 112 & 108 \\
\hline $\operatorname{TrkB}(\mathrm{h})$ & 130 & 141 & 148 & 106 \\
\hline TSSK1(h) & 106 & 102 & 104 & 4 \\
\hline TSSK2(h) & 115 & 120 & 111 & 110 \\
\hline $\operatorname{Txk}(\mathrm{h})$ & 94 & 92 & 100 & 96 \\
\hline ULK2(h) & 100 & 98 & 95 & 93 \\
\hline ULK3(h) & 100 & 101 & 101 & 103 \\
\hline VRK2(h) & 95 & 99 & 107 & 99 \\
\hline WNK2(h) & 130 & 122 & 119 & 106 \\
\hline WNK3(h) & 98 & 109 & 102 & 97 \\
\hline Yes(h) & 102 & 99 & 102 & 96 \\
\hline ZAP-70(h) & 115 & 115 & 110 & 105 \\
\hline ZIPK(h) & 107 & 106 & 112 & 117 \\
\hline
\end{tabular}




\section{VITA}

Jane Elizabeth Craig was born in Baton Rouge, LA in 1992. She earned her Bachelor of Science in Biological Sciences from Louisiana State University with minors in Chemistry and Psychology in 2015. During her time there, she worked in the lab of Steven Hand, studying mitochondrial physiology and cryopreservation of biological membranes. She then joined the College of Graduate Health Sciences (CGHS) at University of Tennessee Health Science Center (UTHSC), studying autophagy regulation at St. Jude Children's Research Hospital. She is expected to receive her Ph.D. in Biomedical Sciences with a concentration in Cancer and Developmental Biology in 2020. 